\title{
Computing the Inversion-Indel Distance
}

\author{
Eyla Willing, Jens Stoye and Marília D. V. Braga
}

\begin{abstract}
The inversion distance, that is the distance between two unichromosomal genomes with the same content allowing only inversions of DNA segments, can be exactly computed thanks to a pioneering approach of Hannenhalli and Pevzner from 1995. In 2000, ElMabrouk extended the inversion model to perform the comparison of unichromosomal genomes with unequal contents, combining inversions with insertions and deletions (indels) of DNA segments, giving rise to the inversion-indel distance. However, only a heuristic was provided for its computation. In 2005, Yancopoulos, Attie and Friedberg started a new branch of research by introducing the generic double cut and join (DCJ) operation, that can represent several genome rearrangements (including inversions). In 2006, Bergeron, Mixtacki and Stoye showed that the DCJ distance can be computed in linear time with a very simple procedure. As a consequence, in 2010 we gave a linear-time algorithm to compute the DCJ-indel distance. This result allowed the inversion-indel model to be revisited from another angle. In 2013, we could show that, when the diagram that represents the relation between the two compared genomes has no bad components, the inversionindel distance is equal to the DCJ-indel distance. In the present work we complete the study of the inversion-indel distance by giving the first algorithm to compute it exactly even in the presence of bad components.
\end{abstract}

Index Terms-Computational biology, genome rearrangements, genomic distance, inversions, reversals, insertions, deletions, indels.

\section{INTRODUCTION}

Genome rearrangement is a branch of comparative genomics that analyses the arrangement of two or more genomes on an abstract level, in which a genome is represented as one (unichromosomal) or more (multichromosomal) ordered sequences of oriented DNA fragments (markers). The structural rearrangements of chromosomal segments, such as inversions, translocations and transpositions, besides fusions and fissions of chromosomes, can change the location (with respect to the chromosome), the order and the orientation of markers. A natural measure of comparison is then the distance, that counts the minimum number of rearrangements required to transform one given genome into another one.

One of the first proposed models aimed to compare unichromosomal genomes (e.g. of bacteria), considering only inversions of contiguous markers. Hannenhalli and Pevzner [1] devised a polynomial time algorithm to compute the inversion distance when each marker occurs exactly once in each genome, based on a diagram that

E. Willing, J. Stoye and M. D. V. Braga are with the Genome Informatics group, Faculty of Technology and Center for Biotechnology (CeBiTec), Bielefeld University, Germany represents the relation between the two given genomes and is composed of cycles only. While for most of the instances based on real data the inversion distance can be obtained by simply counting the number of cycles in this diagram, the complete analysis of the problem requires the computation of other parameters - called bad components - that can be derived from the relational diagram by a procedure that is considerably technical.

The inversion model was then extended to a multichromosomal model including, besides inversions, also fusions and fissions of chromosomes, and translocations, still allowing, with a technically even more complex procedure, the exact computation of the distance in polynomial time when each marker occurs exactly once in each genome [2].

A relaxation on the genome content allows markers that occur in one genome but not in the other, and viceversa. A marker of this type can be added to or removed from a genome by content-modifying operations called insertions and deletions (indels collectively). Combining structural rearrangements and indels is non-trivial if contiguous markers can be inserted or deleted in a single event. Almost 20 years ago, El-Mabrouk [3] studied the inversion model with this relaxation to include indels. However, specially due to its technical complexity, the author could give only a heuristic to compute the inversion-indel distance.

The field of genome rearrangement received a new boost when Yancopoulos, Attie and Friedberg [4] presented a generic model, based on the double cut-and-join $(D C J)$ operation, that allows inversions, translocations, fissions and fusions, and also excisions and integrations of circular chromosomes. It was shown that the DCJ distance can be computed with the help of a surprisingly simple, linear time procedure when each maker occurs exactly once in each genome [5]. The relaxation of the genomes to include indels, besides DCJ operations, increased the technical aspects of the model, but immediately resulted in a linear time exact algorithm to compute the DCJ-indel distance [6], [7].

The potential of using the DCJ model to revisit the inversion model in a more efficient way was first demonstrated in [8]. More recently, the DCJ-indel model was used to give the first exact algorithm to compute the inversion-indel distance in the absence of bad components [9]. In the present work, we complete the study of the inversion-indel model by giving a quadratic time 
algorithm to exactly compute the respective distance even in the presence of bad components. Observe that, since inversions are intrachromosomal rearrangements, the inversion-indel model is restricted to unichromosomal genomes. Our approach is developed considering circular chromosomes, but can be easily generalized to linear chromosomes 1 .

This paper is organized as follows. In Section 2 we show how the relational diagram, with its good and bad components, allows the study of the inversion-indel model and gives a lower bound to the inversion-indel distance. In Section 3 we introduce the tagged component tree, a structure that helps us to handle bad components. In Section 4 we present our main results, effectively showing how to use the tagged component tree to compute the inversion-indel distance. This requires a lengthy, but finite analysis of cases of bad component cutting and merging. In our approach we first safely reduce the tagged component tree to a residual tree with 2 to 10 leaves. Then we enumerate the optimal covers of all possible residual trees. Each possible residual tree corresponds to a topology with a certain leaf composition. Our enumeration includes 56 leaf compositions, each one corresponding to an average of 3 topologies. We present some key examples of the case analysis, explaining the properties that need to be considered. The full enumeration is then given in the appendix. Section 5 concludes with a short summary.

\section{Preliminaries}

Each marker in a chromosome is an oriented DNA fragment. The representation of a marker $g$ in a chromosome $\alpha$ can be the symbol $g$, if it is read in direct orientation in $\alpha$, or the symbol $\bar{g}$, if it is read in reverse orientation. In the present work, duplicated markers are not allowed. We represent a circular chromosome $\alpha$ by a string $s$, obtained by the concatenation of all symbols in $\alpha$, read in any of the two directions (since $\alpha$ is circular, we can start to read it at any marker).

Given two circular chromosomes $\alpha$ and $\beta$, possibly with unequal contents, let $G, A$ and $B$ be three disjoint sets, such that $G$ is the set of common markers that occur once in $\alpha$ and once in $\beta, A$ is the set of markers that occur only in $\alpha$, and $B$ is the set of markers that occur only in $\beta$. The markers in sets $A$ and $B$ are also called unique markers. For example, if we have $\alpha=(a b \bar{x} \bar{c} y d)$ and $\beta=($ asbuvcd), then $G=\{a, b, c, d\}, A=\{x, y\}$ and $B=\{s, u, v\}$.

\subsection{The inversion-indel model}

An inversion is the operation that cuts a circular chromosome at two different positions, creating two linear

1. Linear chromosomes can be artificially "circularized" by the well known technique of capping its two extremities. Fixing the capping of one chromosome, there are only two possible ways of capping the other chromosome. The choice that gives the smaller inversion-indel distance gives the overall result. segments with four open ends, and joins these open ends such that in the new circular chromosome the relative orientation of the linear segments is inverted. Consider, for example, an inversion applied to chromosome $\alpha=(a b \bar{x} \bar{c} y d)$, that cuts before and after $\bar{x} \bar{c}$, creating the segments $\bullet y d a b \bullet$ and $\bullet \bar{x} \bar{c} \bullet$, where the symbol $\bullet$ represents the open ends. If we then join the first with the third and the second with the fourth open end, we obtain $\alpha^{\prime}=(a b c x y d)$, in which the orientation of $\bar{x} \bar{c}$ is inverted with respect to the longer segment.

Since the chromosomes can have unequal marker sets, we also need to consider insertions and deletions of blocks of contiguous markers [3], [6], [7]. We refer to insertions and deletions collectively as indels. Indels have two restrictions [7]: (i) markers of $G$ cannot be deleted; and (ii) an insertion cannot produce duplicated markers. We illustrate an indel with the following example: the deletion of markers $x y$ from chromosome $\alpha^{\prime}=(a b c x y d)$ results in $\alpha^{\prime \prime}=(a b c d)$.

Given two circular chromosomes $\alpha$ and $\beta$, one can transform or sort $\alpha$ into $\beta$ with inversions and indels. We are particularly interested in computing the inversionindel distance, that is denoted by $d_{\mathrm{INV}}^{i d}(\alpha, \beta)$ and corresponds to the minimum number of inversions and indels required to sort $\alpha$ into $\beta$. Observe that, if $|G| \leqslant 1$, the problem of sorting $\alpha$ into $\beta$ becomes trivial: we simply delete at once the unique content of the chromosome of $\alpha$ and insert at once, in the proper orientation, the unique content of the chromosome of $\beta$. Due to this fact, we assume in this work that $|G| \geqslant 2$.

\subsection{Relational diagram}

Given two circular chromosomes $\alpha$ and $\beta$, their relational diagram [10], denoted by $R(\alpha, \beta)$, is a particular view of the master graph [11] and shows the elements of chromosome $\alpha$ in an upper horizontal line and the elements of chromosome $\beta$ in a lower horizontal line. We denote the two extremities of each marker $g \in G$ by $g^{t}$ (tail) and $g^{h}$ (head). For each extremity of $g$ the diagram $R(\alpha, \beta)$ has a vertex in the upper line and a vertex in the lower line. Clearly, each line (that corresponds to one of the two chromosomes) has $2|G|$ vertices, and its vertices are distributed following the same order of the corresponding chromosome. Since the chromosomes are circular, we have to choose one marker $a \in G$ from which we start to read both chromosomes, s.t. in both lines the leftmost vertex is $a^{h}$ and the rightmost is $a^{t}$. Then, for each marker $g \in G$, we connect the upper and the lower vertices that represent $g^{t}$ by a dotted edge. Similarly, we connect the upper and the lower vertices that represent $g^{h}$ by a dotted edge.

Moreover, for each integer $i$ from 1 to $|G|$, let $\gamma_{i}^{1}$ and $\gamma_{i}^{2}$ be the upper vertices (analogously lower vertices) at positions $2 i-1$ and $2 i$ of the corresponding line of the diagram. We connect the upper vertices (analogously lower vertices) $\gamma_{i}^{1}$ and $\gamma_{i}^{2}$ by an $\alpha$-edge (analogously $\beta$ edge) labeled by $\ell$, which is the substring composed of 
the markers of chromosome $\alpha$ (analogously chromosome $\beta$ ) that are between the extremities represented by $\gamma_{i}^{1}$ and $\gamma_{i}^{2}$. We say that $\gamma_{i}^{1}$ and $\gamma_{i}^{2}$ are G-adjacent, that is, they represent extremities of occurrences of markers from $G$ in chromosome $\alpha$ (analogously $\beta$ ), so that in-between only markers from $A$ (analogously $B$ ) can appear. In other words, the label $\ell$ contains no marker of $G$. When the label of an $\alpha$-edge (or a $\beta$-edge) is empty, the edge is said to be clean, otherwise it is said to be labeled.

Each vertex is now connected to one dotted edge and either to one $\alpha$-edge (for the upper vertices) or to one $\beta$ edge (for the lower vertices), thus the degree of each vertex is two and the diagram is simply a collection of cycles. Each cycle alternates a pair ( $\alpha$-edge, dotted edge) with a pair ( $\beta$-edge, dotted edge), consequently the length of each cycle is a multiple of 4 . A cycle that contains at least one labeled edge is said to be labeled, otherwise the cycle is said to be clean. The cycles of $R(\alpha, \beta)$ containing only two dotted edges (and one $\alpha$-edge and one $\beta$-edge) are called 2 -cycles and are said to be inversion-sorted. Longer cycles are inversionunsorted and have to be reduced, by applying inversions, to 2-cycles. This procedure is called inversion-sorting of $\alpha$ into $\beta$ : when the relational diagram of $\alpha$ and $\beta$ is composed exclusively of 2-cycles, the markers from $G$ appear exactly in the same relative order and relative orientations in $\alpha$ and $\beta$ [1].

By walking through each cycle of the relational diagram, arbitrarily in one of the two possible directions, we assign an orientation to each $\alpha$-edge and to each $\beta$-edge. We represent the labels according to the assigned direction instead of taking a simple left-to-right orientation for each edge, in order to avoid any ambiguity. Figure 1 shows a relational diagram of two chromosomes $\alpha$ and $\beta$.

\subsubsection{Bad and good cycles, bad and good components}

The relative orientations of $\alpha$-edges and $\beta$-edges within one cycle are useful for classifying different types of inversions. In the following, without loss of generality, we will refer to operations applied to $\alpha$-edges of $R(\alpha, \beta)$, but a symmetric analysis could be done using $\beta$-edges. Given an inversion $\rho$, the inversion cost of $\rho$, denoted by $\|\rho\|$, depends on the effect of $\rho$ on the number of cycles in $R(\alpha, \beta)$ as follows [1]:

- a split inversion $\rho$ has $\|\rho\|=0$ : it is applied to two $\alpha$ edges that are in the same cycle and have opposite relative orientations, splitting the original cycle into two (increasing the number of cycles in $R(\alpha, \beta)$ by one);

- a neutral inversion $\rho$ has $\|\rho\|=1$ : it is applied to two $\alpha$-edges that are in the same cycle with the same relative orientation, changing the internal organization of the original cycle without splitting it;

- a joint inversion $\rho$ has $\|\rho\|=2$ : it is applied to $\alpha$-edges from two distinct cycles $C_{1}$ and $C_{2}$, joining $C_{1}$ and $\mathrm{C}_{2}$ into one cycle (decreasing the number of cycles in $R(\alpha, \beta)$ by one).
If a cycle $C$ does not have a pair of $\alpha$-edges with opposite orientations, it cannot be split by any inversion applied to its $\alpha$-edges and is called a bad cycle. Otherwise the cycle $C$ is said to be good. Two distinct cycles $C$ and $C^{\prime}$ are said to be interleaving when in the relational diagram there is at least one $\alpha$-edge of $C$ between two $\alpha$-edges of $C^{\prime}$ and at least one $\alpha$-edge of $C^{\prime}$ between two $\alpha$-edges of $C$. An interleaving path connecting two cycles $C$ and $C^{\prime}$ is defined as a sequence of cycles $C_{1}, C_{2}, \ldots, C_{k}$ such that $C_{1}=C, C_{k}=C^{\prime}$ and $C_{i}$ and $C_{i+1}$ are interleaving for all $i$, $1 \leqslant i<k$. An interleaving component or simply component is then a maximal set of cycles $\mathcal{K}$ where each $C \in \mathcal{K}$ is connected by an interleaving path to any other $C^{\prime} \in \mathcal{K}$.

Components can be of three types. The first type is a 2-cycle, that can never interleave with any other cycle and is called a trivial component. The other two types are components of inversion-unsorted cycles. A nontrivial component that contains at least one good cycle can be sorted with split inversions and is called a good component, otherwise it is called a bad component [1]. The relational diagram represented in Figure 11 has six components: one good (the cycle $C_{2}$ ), two trivial (the cycles $C_{4}$ and $C_{7}$ ) and three bad (each of the cycles $C_{1}$ and $C_{3}$ and interleaving cycles $C_{5}$ and $\left.C_{6}\right)$. Let $d_{\mathrm{INV}}(\alpha, \beta)$ be the minimum number of inversions required to transform $R(\alpha, \beta)$ into a collection of inversion-sorted 2-cycles. It is well known that when $R(\alpha, \beta)$ has no bad components, this transformation can be done by applying split inversions only [1]. Since the maximum number of cycles in $R(\alpha, \beta)$ is equal to $|G|$, we have:

Lemma 1 (adapted from [1], [12]): For two circular chromosomes $\alpha$ and $\beta$, such that $R(\alpha, \beta)$ has no bad component, $d_{\mathrm{INV}}(\alpha, \beta)=|G|-c$, where $c$ is the number of cycles in $R(\alpha, \beta)$.

\subsubsection{Runs, indel-potential and inversion-indel distance lower bound}

Given two chromosomes $\alpha$ and $\beta$ and a cycle $C$ of $R(\alpha, \beta)$, a run is a maximal subpath of $C$, in which the first and the last edges are labeled and all labeled edges belong to the same chromosome [7]. A run with labels in chromosome $\alpha$ is also called an $A$-run, and a run with labels in chromosome $\beta$ is called a $B$-run. Observe that, since we start counting runs in any border between an $A$ - and a $B$-run, a cycle can have either 0 , or 1 , or an even number of runs. We denote by $\Lambda(C)$ the number of runs in cycle $C$. As an example, note that the cycle $C_{1}$ represented in Figure 1 has 2 runs $\left(\left\{a^{h} t j^{t}, j^{t} i^{h}, i^{h} u k^{t}\right\}\right.$ is an $A$-run, while $\left\{k^{t} \bar{y} j^{h}, j^{h} b^{t}, b^{t} \bar{w} a^{h}\right\}$ is a $B$-run). While doing the inversion-sorting of good components with split inversions only, an entire run can be accumulated into a single edge of the relational diagram [7], [9]. The number $\Lambda(C)$ is then an upper bound for the contribution of cycle $C$ in the final number of indels obtained while doing the inversion-sorting of $R(\alpha, \beta)$ with split inversions only. The exact value of this contribution is the indel-potential of a cycle $C$, that is denoted by $\lambda(C)$ and can be directly derived from $\Lambda(C)$ as follows: 


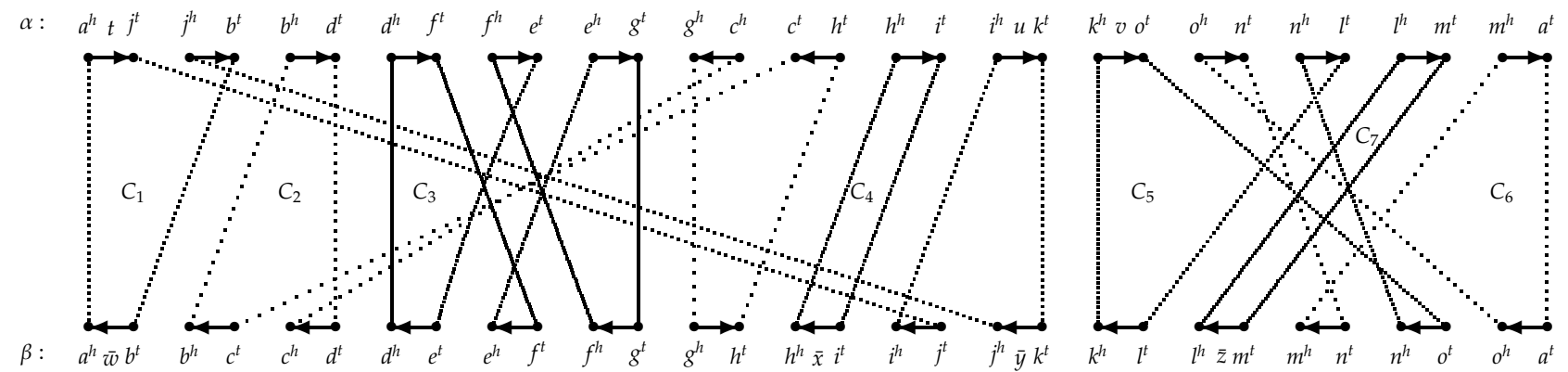

Fig. 1. For chromosomes $\alpha=$ (atjbdfegc̄hiukvonlm) and $\beta=$ (awbcdefghxijyklzmno), with sets of common and unique markers $G=\{a, b, c, d, e, f, g, h, i, j, k, l, m, n, o\}, A=\{t, u, v\}$ and $B=\{w, x, y, z\}$, the relational diagram has the five inversion-unsorted cycles $C_{1}, C_{2}, C_{3}, C_{5}$ and $C_{6}$ and the two inversion-sorted cycles $C_{4}$ and $C_{7}$. Cycles $C_{1}, C_{4}, C_{5}$ and $C_{7}$ are labeled and cycles $C_{2}, C_{3}$ and $C_{6}$ are clean.

Proposition 1 (adapted from [7]): Given two circular chromosomes $\alpha$ and $\beta$, the indel-potential of a cycle $C$ of $R(\alpha, \beta)$ is given by

$$
\lambda(C)= \begin{cases}\Lambda(C), & \text { if } \Lambda(C) \in\{0,1,2\}, \\ \frac{\Lambda(C)}{2}+1, & \text { if } \Lambda(C) \in\{4,6,8, \ldots\} .\end{cases}
$$

Let $\lambda_{0}$ and $\lambda_{1}$ be, respectively, the sum of the indelpotentials for the components of the relational diagram before and after an inversion $\rho$. The indel-cost of $\rho$ is then $\Delta \lambda(\rho)=\lambda_{1}-\lambda_{0}$, and the inversion-indel cost of $\rho$ is defined as $\Delta d(\rho)=\|\rho\|+\Delta \lambda(\rho)$. If $R(\alpha, \beta)$ has good cycles, it is always possible to find a split inversion $\rho$ such that $\rho$ does not increase the overall indel-potential of $R(\alpha, \beta)$, that is, $\Delta \lambda(\rho)=0$ and, consequently, $\Delta d(\rho)=$ $0[9]$. Moreover, it has been shown that using neutral or joint inversions one can never achieve $\Delta d<0$. Therefore, we have the following result:

Theorem 1 (from [7], [9]): Given two circular chromosomes $\alpha$ and $\beta$, such that $R(\alpha, \beta)$ has $c$ cycles and no bad component, we have

$$
d_{\mathrm{INV}}^{\text {id }}(\alpha, \beta)=|G|-c+\sum_{C \in R(\alpha, \beta)} \lambda(C) .
$$

\section{Handing bad components With the tagged com- PONENT TREE}

When $R(\alpha, \beta)$ has bad components, it requires additional neutral and joint inversions to be sorted. Therefore, in this case the formula given in Theorem 1 is only a lower bound for the inversion-indel distance. Denoting by $\tau^{*}(\alpha, \beta)$ the extra cost of optimally combining neutral and joint inversions to sort the bad components of $R(\alpha, \beta)$ and given that $c$ is the number of cycles in $R(\alpha, \beta)$, we can express the complete formula for the inversion-indel distance as:

$$
d_{\mathrm{INV}}^{i d}(\alpha, \beta)=|G|-c+\sum_{C \in R(\alpha, \beta)} \lambda(C)+\tau^{*}(\alpha, \beta) .
$$

\subsection{Neutral and joint inversions sort bad compo- nents}

For computing $\tau^{*}(\alpha, \beta)$ it is necessary to examine the possible values of the inversion-indel cost $\Delta d$ for neutral and joint inversions. In the remainder of this section we will recall results from [1], [9], explaining the approach of sorting bad components with neutral and joint inversions that minimize indels. Before that, we need to introduce the following notation. A cycle with no run is represented by $\bigcirc$. A cycle with exactly one $A$-run (respectively one $B$-run) is represented by $\bigcirc_{A}$ (respectively $\left.\bigcirc_{B}\right)$. Similarly, a cycle with $2 i$ runs is represented by $\bigcirc_{A B}^{i}$. For simplicity, we denote $\bigcirc_{A B}^{1}$ by $\bigcirc_{A B}$.

\subsubsection{Neutral inversions cut bad components}

Let $\mathcal{K}$ be a bad component and let $C$ be any (bad) cycle in $\mathcal{K}$. A neutral inversion, applied to $C$, turns $\mathcal{K}$ into a good component [1]. This type of inversion is said to be a cut of the bad component. If $R(\alpha, \beta)$ has a bad cycle of type $\bigcirc_{A B}^{i}$ with $i \geqslant 2$, it is always possible to apply a neutral inversion $\rho$ such that we obtain a good cycle of type $\bigcirc_{\mathrm{AB}}^{i-1}$. In other words, two $A$-runs and two $B$-runs are merged by $\rho$, implying $\Delta \lambda(\rho)=-1$ and, consequently, $\Delta d(\rho)=0$. For all other types of bad cycles, it is not possible to decrease the overall indel-potential with neutral inversions [9].

Observation 1: Since it is always possible to apply a costless neutral inversion on a cycle of type $\bigcirc_{\mathrm{AB}}^{i}$ with $i \geqslant 2$, we can assume that $R(\alpha, \beta)$ has no cycle of this type, that is, we can assume that any cycle in $R(\alpha, \beta)$ has at most 2 runs. In this setting, the most efficient way to cut a bad component is by applying a neutral inversion $\rho$ with $\Delta d(\rho)=1$.

\subsubsection{Joint inversions merge bad components}

Let $\rho$ be a joint inversion applied to two distinct cycles $C_{1}$ and $C_{2}$, resulting in a single cycle $C$. Observe that the cycle $C$ is always good, even when both $C_{1}$ and $C_{2}$ are bad. Moreover, if $C_{1}$ and $C_{2}$ belong to two distinct components $\mathcal{K}_{1}$ and $\mathcal{K}_{2}$, these components are merged into a single good component $\mathcal{K}$ that contains the good cycle $C$ [1]. Actually, an inversion $\rho$ has the potential of 
TABLE 1

Inversion-indel costs of distinct types of joint inversions (any joint inversion $\rho$ has inversion cost $\|\rho\|=2$, a cycle with any number of runs is represented by $\bigcirc_{*}$ )

\begin{tabular}{cccc}
\hline sources & resultant & $\boldsymbol{\Delta} \boldsymbol{\lambda}(\boldsymbol{\rho})$ & $\boldsymbol{\Delta} \boldsymbol{d}(\boldsymbol{\rho})$ \\
\hline$\bigcirc^{+} \bigcirc_{*}$ & $\bigcirc_{*}$ & 0 & 2 \\
$\bigcirc_{\mathrm{A}}+\bigcirc_{\mathrm{B}}$ & $\bigcirc_{\mathrm{AB}}$ & 0 & 2 \\
$\bigcirc_{\mathrm{A}}+\bigcirc_{\mathrm{A}}$ & $\bigcirc_{\mathrm{A}}$ & -1 & 1 \\
$\bigcirc_{\mathrm{B}}+\bigcirc_{\mathrm{B}}$ & $\bigcirc_{\mathrm{B}}$ & -1 & 1 \\
$\bigcirc_{\mathrm{A}}+\bigcirc_{\mathrm{AB}}^{i}$ & $\bigcirc_{\mathrm{AB}}^{i}$ & -1 & 1 \\
$\bigcirc_{\mathrm{B}}+\bigcirc_{\mathrm{AB}}^{i}$ & $\bigcirc_{\mathrm{AB}}^{i}$ & -1 & 1 \\
$\bigcirc_{\mathrm{AB}}^{i}+\bigcirc_{\mathrm{AB}}^{i^{\prime}}$ & $\bigcirc_{\mathrm{AB}}^{\left(i+i^{\prime}-1\right)}$ & -2 & 0 \\
\hline
\end{tabular}

merging even more than two components at once, as we will describe in the following. Without loss of generality, let us assume that, if $R(\alpha, \beta)$ has at least two components, the first and the last $\alpha$-edges of $R(\alpha, \beta)$ belong to two distinct components.

Let $\mathcal{K}_{1}, \mathcal{K}_{2}$ and $\mathcal{K}_{3}$ be three distinct components in $R(\alpha, \beta)$ such that if we take the rightmost $\alpha$-edge of $\mathcal{K}_{1}$ and look at the following $\alpha$-edges one by one, we find an edge of $\mathcal{K}_{3}$ before finding an edge of $\mathcal{K}_{2}$. In the same way, if we take the rightmost $\alpha$-edge of $\mathcal{K}_{2}$ and look at the following $\alpha$-edges one by one, we find an edge of $\mathcal{K}_{3}$ before finding an edge of $\mathcal{K}_{1}$. The component $\mathcal{K}_{3}$ is then said to separate $\mathcal{K}_{1}$ and $\mathcal{K}_{2}$. (In Figure 1 the good component $\left\{C_{1}\right\}$ separates the good component $\left\{C_{2}\right\}$, the bad component $\left\{C_{3}\right\}$ and the trivial component $\left\{C_{4}\right\}$ from the bad component $\left\{C_{5}, C_{6}\right\}$ and the trivial component $\left\{C_{7}\right\}$. Similarly, the bad component $\left\{C_{5}, C_{6}\right\}$ separates $\left\{C_{7}\right\}$ from $\left\{C_{1}\right\},\left\{C_{2}\right\},\left\{C_{3}\right\}$ and $\left\{C_{4}\right\}$.) By joining with an inversion $\rho$ two cycles $C_{1}$ and $C_{2}$, that belong to two distinct components $\mathcal{K}_{1}$ and $\mathcal{K}_{2}$ respectively, we merge not only the components $\mathcal{K}_{1}$ and $\mathcal{K}_{2}$, but also all components that separate $\mathcal{K}_{1}$ and $\mathcal{K}_{2}$, into a single component $\mathcal{K}$. Even when all merged components are bad, the new component $\mathcal{K}$ is always good [1]. A joint inversion can then be used to merge two or more bad components. Recall that any joint inversion $\rho$ has $\|\rho\|=2$, therefore $\Delta d(\rho)=2+\Delta \lambda(\rho)$. Table 1 enumerates the inversionindel costs of the different types of joint inversions.

Observation 2: Since it is always possible to apply a costless joint inversion on two cycles of type $\bigcirc_{\mathrm{AB}}^{i}$, we assume that $R(\alpha, \beta)$ has at most one cycle of this type and that any joint inversion $\rho$ merging components of $R(\alpha, \beta)$ has $\Delta d(\rho) \in\{1,2\}$.

Observations 1 and 2 imply that we can assume that $R(\alpha, \beta)$ has at most one cycle of type $\bigcirc_{A B}$ and no cycle of type $\bigcirc_{A B}^{i}$ with $i \geqslant 2$. This assures that a cut of a bad component costs always 1 and that merging bad components costs either 1 or 2 .

\subsection{Tagged component tree}

The component separation described above can be identified by an alternative structure, that represents the chaining and nesting relationships of the components and helps us to find the best way of cutting and merging bad components of $R(\alpha, \beta)$.

\subsubsection{Chained tagged component tree}

Let a chain be a sequence of components $\mathcal{K}_{1}, \mathcal{K}_{2}, \ldots, \mathcal{K}_{n}$ for which the rightmost $\alpha$-edge of $\mathcal{K}_{i}$ is succeeded by the leftmost $\alpha$-edge of $\mathcal{K}_{i+1}$, for $1 \leqslant i<n$. A chain is maximal when it cannot be extended to the left nor to the right. A maximal chain $\mathcal{H}$ is nested in a component $\mathcal{K}$ when the leftmost $\alpha$-edge of $\mathcal{H}$ is preceded by an $\alpha$-edge of $\mathcal{K}$ and the rightmost $\alpha$-edge of $\mathcal{H}$ is succeeded by an $\alpha$-edge of $\mathcal{K}$.

We can then build the chained tagged component tree $T_{\square}(\alpha, \beta)$ as follows [8], [9], [13]:

1) Each component $\mathcal{K}$ from $R(\alpha, \beta)$ is represented by a round node $v$. If $\mathcal{K}$ is a bad component, then $v$ is a bad node, drawn in white. Otherwise $v$ is a good node, drawn in black. A good node can represent a trivial or a good component. Each round node $v$ has a tag set $\operatorname{TAG}(v)$, initially empty, that can receive at most two tags: (i) the $\operatorname{tag} \mathbf{A}$ is added to TAG(v) if at least one cycle of the component $\mathcal{K}$ has an $A$ run; and (ii) the tag $\mathbf{B}$ is added to $\operatorname{TAG}(v)$ if at least one cycle of $\mathcal{K}$ has a $B$-run.

2) Each maximal chain is represented by a square node whose children are the round nodes that represent the components of this chain. A square node is either the root or a child of the component in which this chain is nested.

The chained tagged component tree $T_{\square}(\alpha, \beta)$ is the same component tree as introduced in [13], except that here we add the tag sets. This tree has the following important property. Let $P$ be a path connecting two distinct round nodes $u_{1}$ and $u_{2}$ in $T_{\square}(\alpha, \beta)$. The round nodes in $P \backslash\left\{u_{1}, u_{2}\right\}$ correspond exactly to the components that separate $u_{1}$ and $u_{2}$ in $R(\alpha, \beta)$.

\subsubsection{Max-flower contraction}

Let a max-flower of a chained tagged component tree be a maximal connected subgraph composed of good and/or square nodes only. Suppose we have a max-flower $f$ of a chained tagged component tree $T$. The contraction of $f$ in $T$ consists of two steps:

1) Replace $f$ by a single good round node $g$, such that $g$ is connected to all bad nodes connected to $f$ and $\operatorname{TAG}(g)$ is the union of tag sets of all nodes from $f$ (assuming that square nodes have empty tag sets);

2) If $g$ has an empty tag set and exactly two neighbors $b_{1}$ and $b_{2}, g$ is removed from the tree and $b_{1}$ is directly connected to $b_{2}$; otherwise, if $g$ is a leaf and $b$ is the bad node connected to $g$, the tag set $\operatorname{TAG}(g)$ is added to $\operatorname{TAG}(b)$ and $g$ is removed from the tree. 
After repeating this procedure for all (disjoint) maxflowers we obtain an unrooted tagged component tree $T^{\prime}$, that is said to be flower-contracted, that is, the tree $T^{\prime}$ has no square nodes, no good leaves, no good nodes of degree 2 with an empty tag set, and each remaining max-flower of $T^{\prime}$ corresponds to a single good node. As we will see in the next subsection, the computation of $\tau^{*}(\alpha, \beta)$ is derived from the analysis of an unrooted tagged component tree that is exactly the flowercontracted version of the chained tagged component tree $T_{\square}(\alpha, \beta)$ and is denoted by $T_{\circ}(\alpha, \beta)$. See examples of its construction in Figure 2.
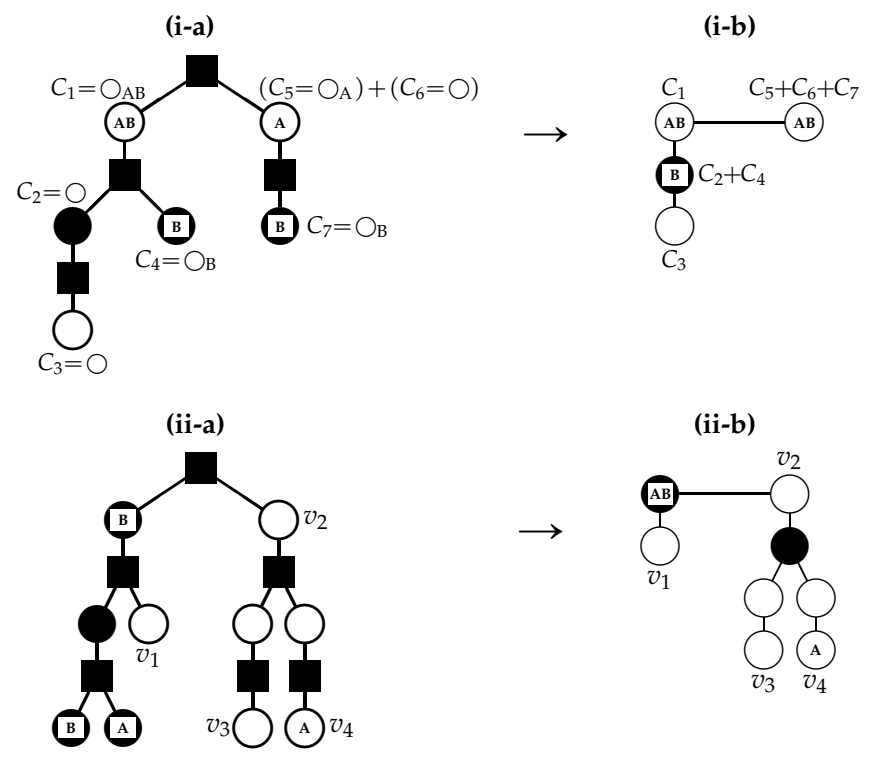

(ii-b)

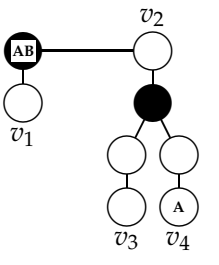

Fig. 2. (i-a) The chained tagged component tree $T_{\square}(\alpha, \beta)$ derived from the relational diagram represented in Figure 1 with three bad (white) and three good (black) nodes and their respective tags. (i-b) The tree $T_{\circ}(\alpha, \beta)$ obtained from $T_{\square}(\alpha, \beta)$. (ii-a,b) Another example illustrating the max-flower contraction.

\subsection{Cover and optimal cover}

The diagram $R(\alpha, \beta)$ can have any number of good and bad cycles of types $\bigcirc, \bigcirc_{A}$ and $\bigcirc_{B}$. Due to Observations 1 and 2. we assume that $R(\alpha, \beta)$ has at most one cycle of type $\bigcirc_{A B}$ and no cycle of type $\bigcirc_{A B}^{i}$ with $i \geqslant 2$. Therefore, the cost of cutting a component is always one, independently of the number of tags of the corresponding node in $T_{\circ}(\alpha, \beta)$ and - even if a node of $T_{\circ}(\alpha, \beta)$ has both tags at most one of them can be "used" in each joint inversion merging components, that costs either 1 or 2.

Similar to [13], in our analysis we focus on paths covering the bad nodes of $T_{\circ}(\alpha, \beta)$. We denote by $\tau(P)$ the cost of a path $P$, that can be of two types. The path $P$ is short if it contains a single bad node. It corresponds to the cut of the component represented by its bad node and has therefore cost $\tau(P)=1$. On the other hand, the path $P$ is long if it contains at least two bad nodes. It corresponds to the merging of all components represented by its nodes, by a joint inversion applied to two cycles, each of the two belonging to one of the two endpoints of the path. The cost of $P$ is therefore either $\tau(P)=1$, if its endpoints share at least one tag (indel-saving path), or $\tau(P)=2$ otherwise (indel-neutral path).

A cover of $T_{\circ}(\alpha, \beta)$ is defined as a set of paths $\mathcal{P}$ such that each bad node of $T_{0}(\alpha, \beta)$ is contained in at least one path $P \in \mathcal{P}$. The cost of $\mathcal{P}$ is given by $\tau(\mathcal{P})=\sum_{P \in \mathcal{P}} \tau(P)$. If $\tau(\mathcal{P})$ is minimum, $\mathcal{P}$ is an optimal cover and $\tau(\mathcal{P})=$ $\tau\left(T_{\circ}(\alpha, \beta)\right)=\tau^{*}(\alpha, \beta)$ [8], [9].

The reason behind contracting the tree to get one with the minimum number of good nodes and whose leaves are bad nodes is simply to get a more compact tree, while preserving the same bad nodes and tags of the original tree. In other words, since the flowercontractions preserve in $T_{\circ}(\alpha, \beta)$ all the tags from $T_{\square}(\alpha, \beta)$ in the same relative positions with respect to the bad nodes, we have $\tau\left(T_{\circ}(\alpha, \beta)\right)=\tau\left(T_{\square}(\alpha, \beta)\right)$.

\subsubsection{Covering a tree with traversals}

Given a tree $T$ with $\ell$ leaves, a leaf-branch of $T$ is: (i) either the complete tree $T$, if $\ell \leqslant 2$; (ii) or, if $\ell \geqslant 3$, a maximal path $u_{1}, u_{2}, \ldots, u_{k}$, such that $u_{1}$ is a leaf of $T$ and, for $i=2, \ldots, k$, the degree of internal node $u_{i}$ in $T$ is two. The second case includes a leaf-branch consisting of a leaf only, if that is directly connected to a branching node of $T$.

A path $P$ whose both endpoints are leaves is called a traversal. Observe that, if $\ell \geqslant 2$ is the number of leaves of a tagged component tree $T_{\circ}(\alpha, \beta)$, the minimum number of traversals required to cover all $\ell$ leaves is $\left\lceil\frac{\ell}{2}\right\rceil$. Indeed, any unrooted tree with at least two leaves can be completely covered with this minimum number of traversals. If the number of leaves $\ell$ is even, the traversals can be obtained with a very simple procedure (Algorithm 1, from [14]). If $\ell$ is odd, it is necessary to remove one leaf-branch from the tree, run Algorithm 1 and add one extra traversal connecting the removed leafbranch and any other leaf.

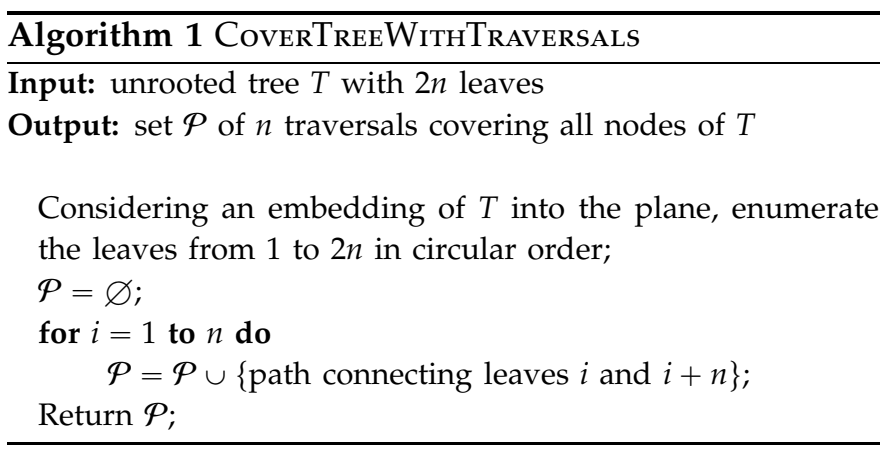

The fact that Algorithm 1 covers the complete tree proves the following theorem.

Theorem 2 (Adapted from [14]): Given a tree $T$ with $2 n$ leaves, it is always possible to find a vertex $v$ in $T$, such that there is a cover of $T$ composed of $n$ traversals, all 
of whom containing the vertex $v$. Such a vertex is called a balanced vertex of $T$.

Proof given in [14]: Considering any two paths in the set returned by Algorithm 1, their endpoints alternate along the circle which contains the leaves in increasing order. Therefore these two paths clearly intersect each other. If in a tree a set of paths does not contain two disjoint paths, then all the paths share a common vertex $v$ (a proof is given in [15]). And because these paths connect $v$ to the leaves, they cover all edges of the tree.

\subsubsection{The simplest instances give a lower and an upper bound}

A node of the tagged component tree $T_{\circ}(\alpha, \beta)$ whose tag set is empty is called clean, otherwise it is tagged. We will now give solutions for the simplest instances of the problem of computing the cost $\tau^{*}(\alpha, \beta)$ of an optimal cover of $T_{\circ}(\alpha, \beta)$, that are: (i) the case in which all leaves are tagged and share at least one tag and (ii) the case in which all leaves are clean. These two cases correspond, respectively, to tight lower and upper bounds for $\tau^{*}(\alpha, \beta)$ and are based on optimal covers composed of traversals and at most one short path.

Theorem 3: Let all $\ell \geqslant 2$ leaves of a tagged component tree $T_{\circ}(\alpha, \beta)$ share at least one tag. The cost of any optimal cover of $T_{\circ}(\alpha, \beta)$ is $\tau^{*}(\alpha, \beta)=\left\lceil\frac{\ell}{2}\right\rceil$.

Proof: Any traversal is indel-saving (costs 1 ) because the leaves of $T_{\circ}(\alpha, \beta)$ share a tag. As we have already explained before, with Algorithm 1 we can find $\left\lceil\frac{\ell}{2}\right\rceil$ traversals covering $T_{\circ}(\alpha, \beta)$ completely.

When the tree has clean leaves, the length of the leafbranches can influence the cost of an optimal cover. A leaf-branch of the tree $T_{\circ}(\alpha, \beta)$ is long if it contains at least two bad nodes, otherwise it is short.

Theorem 4: Let all $\ell \geqslant 2$ leaves of a tagged component tree $T_{\circ}(\alpha, \beta)$ be clean. The cost of any optimal cover of $T_{\circ}(\alpha, \beta)$ is

$$
\tau^{*}(\alpha, \beta)= \begin{cases}\ell+1 & \text { if } \ell \text { is odd and all leaf-branches } \\ & \text { are long ("fortress" [1]), } \\ \ell & \text { otherwise. }\end{cases}
$$

Proof: Any traversal is indel-neutral (costs 2) because the leaves of $T_{\circ}(\alpha, \beta)$ are clean. When $\ell$ is even, with Algorithm 1 we can cover the tree with a set of $\frac{\ell}{2}$ traversals, giving an optimal cost of $\ell$ in this case. When $\ell$ is odd, let $S$ be a short leaf-branch of $T_{\circ}(\alpha, \beta)$, if there is any, otherwise any of the long leaf-branches. The subtree obtained from $T_{0}(\alpha, \beta)$ by removing the leaf-branch $S$ can be covered by a set of $\frac{\ell-1}{2}$ traversals obtained wit Algorithm 1. Then, if $S$ is a short leaf-branch, it can be covered by a short path, giving an optimal cost of $\ell-1+1=\ell$. Otherwise, $S$ is a long leaf-branch and we have the classical situation of a "fortress" [1], in which $S$ can only be covered by a long path with cost 2 , resulting in the optimal cost of $\ell-1+2=\ell+1$.

\section{Obtaining an optimal cover of trees with at LEAST TWO LEAVES THAT SHARE NO TAG}

In this section we will describe our approach of finding an optimal cover of all intermediate and more intrincate instances of the tagged component tree.

Given a path $P$, a P-reduction $T_{\circ}^{-P}(\alpha, \beta)$ of $T_{\circ}(\alpha, \beta)$ results in a smaller tagged component tree as follows. Let $T^{\prime}$ be the tagged tree obtained from $T_{\circ}(\alpha, \beta)$ by replacing each bad node $b$ of $P$ by a good node $g$, such that $\operatorname{TAG}(g)=\operatorname{TAG}(b)$. Then $T_{\circ}^{-P}(\alpha, \beta)$ is the flowercontracted version of $T^{\prime}$. A $P$-reduction is said to be safe if $\tau\left(T_{\circ}(\alpha, \beta)\right)=\tau\left(T_{\circ}^{-P}(\alpha, \beta)\right)+\tau(P)$, otherwise it is unsafe. We will build optimal covers by first "simplifying" the tagged component tree with safe reductions.

From now on we will denote the three possible nonempty tag sets $\{A\},\{B\}$ and $\{A, B\}$, respectively, simply by $\mathrm{A}, \mathrm{B}$ and $\mathrm{AB}$. We can then refer to $\mathrm{A}-, \mathrm{B}-, \varnothing-$ - or $\mathrm{AB}-$ nodes or -leaves, depending on the respective set of tags. Tagged component trees and their optimal covers will be shown in figures with the following notation:

- Leaves will be usually labeled as described: (i) $a, a_{1}$, $a_{2}$, etc., will be labels of A-leaves; (ii) $b, b_{1}, b_{2}$, etc., will be labels of B-leaves; (iii) $x, x_{1}, x_{2}$, etc., will be labels of AB-leaves; and (iv) $s$ is the label of the solo leaf (defined in Section 4.2.1), while $c, c_{1}, c_{2}$, etc., will be labels of other clean leaves.

- In the given optimal covers, traversals will be represented by the symbol $\circ \circ$, semi-traversals (defined in Section 4.1.6) will be represented by the symbol oand the dots above these symbols will indicate the cost of each path.

\subsection{Properties of tagged component tree topologies and their covers}

We need to describe the possible topologies of tagged component trees in order to devise our approach of simplifying the tree and finding optimal covers.

\subsubsection{Subtrees and partition subtrees}

Let $\mathcal{N}$ be a set of nodes of $T_{\circ}(\alpha, \beta)$. Then $\Upsilon(\mathcal{N})$ denotes the subtree induced by $\mathcal{N}$, i. e., the smallest connected subtree of $T_{\circ}(\alpha, \beta)$ containing all the nodes from $\mathcal{N}$. The leaves of $T_{\circ}(\alpha, \beta)$ can be partitioned into four disjoint classes $L_{A}, L_{B}, L_{\varnothing}$ and $L_{A B}$, that are, respectively, the sets of A-leaves, B-leaves, $\varnothing$-leaves and AB-leaves of $T_{\circ}(\alpha, \beta)$. A partition subtree is then a subtree $\Upsilon(\mathcal{L})$ where $\mathcal{L}$ is the union of one to four leaf classes. In general, the topology and connections between the possible partition subtrees drive our approach of finding an optimal cover for the tree $T_{\circ}(\alpha, \beta)$. The four partition subtrees $\Upsilon\left(L_{\mathrm{A}}\right)$, $\Upsilon\left(L_{\mathrm{B}}\right), \Upsilon\left(L_{\varnothing}\right)$ and $\Upsilon\left(L_{\mathrm{AB}}\right)$ are called canonical subtrees of $T_{\circ}(\alpha, \beta)$. While canonical subtree $\Upsilon\left(L_{\varnothing}\right)$ is said to be clean, subtrees $\Upsilon\left(L_{\mathrm{A}}\right), \Upsilon\left(L_{\mathrm{B}}\right)$ and $\Upsilon\left(L_{\mathrm{AB}}\right)$ are said to be tagged. 


\subsubsection{Complementary subtree}

Let $\Upsilon(\mathcal{L})$ be a partition subtree. The complementary subtree of $\Upsilon(\mathcal{L})$, denoted by $\bar{\Upsilon}(\mathcal{L})$, is the partition subtree $\Upsilon\left(\mathcal{L}^{\prime}\right)$, where $\mathcal{L}^{\prime}$ is the set composed of all leaves of $T_{\circ}(\alpha, \beta)$ except those from $\mathcal{L}$. Complementary subtree is a reciprocal concept, thus $\bar{\Upsilon}\left(\mathcal{L}^{\prime}\right)$ is the subtree $\Upsilon(\mathcal{L})$ itself. For example, considering the canonical subtree $\Upsilon\left(L_{\mathrm{A}}\right)$, its complementary subtree is $\bar{\Upsilon}\left(L_{\mathrm{A}}\right)=\Upsilon\left(L_{\mathrm{B}} \cup L_{\mathrm{AB}} \cup L_{\varnothing}\right)$. Another example is the partition subtree $\Upsilon\left(L_{B} \cup L_{\varnothing}\right)$, whose complementary subtree is $\bar{\Upsilon}\left(L_{\mathrm{B}} \cup L_{\varnothing}\right)=\Upsilon\left(L_{\mathrm{A}} \cup L_{\mathrm{AB}}\right)$. Note that $\bar{\Upsilon}(\mathcal{L})$ and $T_{\circ}(\alpha, \beta) \backslash \Upsilon(\mathcal{L})$ can be distinct and, while the first is necessarily a tree, the second can be a forest.

\subsubsection{Links and bad links}

If $\Upsilon\left(\mathcal{L}_{1}\right)$ and $\Upsilon\left(\mathcal{L}_{2}\right)$ are two non-empty and disjoint partition subtrees, there is exactly one path connecting $\Upsilon\left(\mathcal{L}_{1}\right)$ to $\Upsilon\left(\mathcal{L}_{2}\right)$ in $T_{\circ}(\alpha, \beta)$. This path is called the link between $\Upsilon\left(\mathcal{L}_{1}\right)$ and $\Upsilon\left(\mathcal{L}_{2}\right)$. A link is a bad link when it contains at least one bad node, otherwise it is a good link. A bad link that contains two or more bad nodes is called long bad link. Otherwise, if it contains a single bad node, it is called short bad link.

\subsubsection{Co-rooted and separated partition subtrees}

Two non-empty partition subtrees $\Upsilon\left(\mathcal{L}_{1}\right)$ and $\Upsilon\left(\mathcal{L}_{2}\right)$ are said to be separated when they are disjoint and connected by a bad link. Otherwise, they are co-rooted. A partition subtree $\Upsilon(\mathcal{L})$ is said to be isolated if it is separated from its complementary subtree $\bar{\Upsilon}(\mathcal{L})$, and non-isolated if it is co-rooted with its complementary subtree $\bar{\Upsilon}(\mathcal{L})$. If each canonical subtree is non-isolated and, in addition, the partition subtree $\Upsilon\left(L_{\mathrm{A}} \cup L_{\mathrm{B}}\right)$ is non-isolated, then $T_{\circ}(\alpha, \beta)$ is said to be fully co-rooted. At the other extreme, if each canonical subtree $\Upsilon(L)$ is isolated and, in addition, for a tree with four leaf types, either the subtree $\Upsilon\left(L_{A} \cup L_{B}\right)$, or the subtree $\Upsilon\left(L_{\mathrm{A}} \cup L_{\varnothing}\right)$, or the subtree $\Upsilon\left(L_{\mathrm{A}} \cup L_{\mathrm{AB}}\right)$ is isolated, then $T_{\circ}(\alpha, \beta)$ is said to be fully separated. Examples of various topologies are given in Figure 3.

\subsubsection{In-, out- and semi-traversals}

Let $u_{1}$ and $u_{2}$ be two leaves of $T_{\circ}(\alpha, \beta)$. If both $u_{1}$ and $u_{2}$ are in the same canonical subtree $\Upsilon(L)$, the traversal between $u_{1}$ and $u_{2}$ is said to be an in-traversal (of the canonical subtree $\Upsilon(L)$ ). On the other hand, if $u_{1}$ and $u_{2}$ are in distinct canonical subtrees $\Upsilon\left(L_{1}\right)$ and $\Upsilon\left(L_{2}\right)$, the traversal between $u_{1}$ and $u_{2}$ is said to be an out-traversal (between the canonical subtrees $\Upsilon\left(L_{1}\right)$ and $\Upsilon\left(L_{2}\right)$ ). Note that in-traversals of tagged canonical subtrees are indelsaving (cost 1), while in-traversals of the clean canonical subtree are indel-neutral (cost 2). Out-traversals are usually indel-neutral, except in the case of one subtree being $\Upsilon\left(L_{\mathrm{AB}}\right)$ and the other subtree being either $\Upsilon\left(L_{\mathrm{A}}\right)$ or $\Upsilon\left(L_{\mathrm{B}}\right)$, when they are indel-saving.

Now let $u_{1}$ be a leaf of a canonical subtree $\Upsilon(L)$ and $u_{2}$ be an internal node of $T_{\circ}(\alpha, \beta)$ that is not in $\Upsilon(L)$. The path connecting $u_{1}$ and $u_{2}$ is called a semi-traversal,
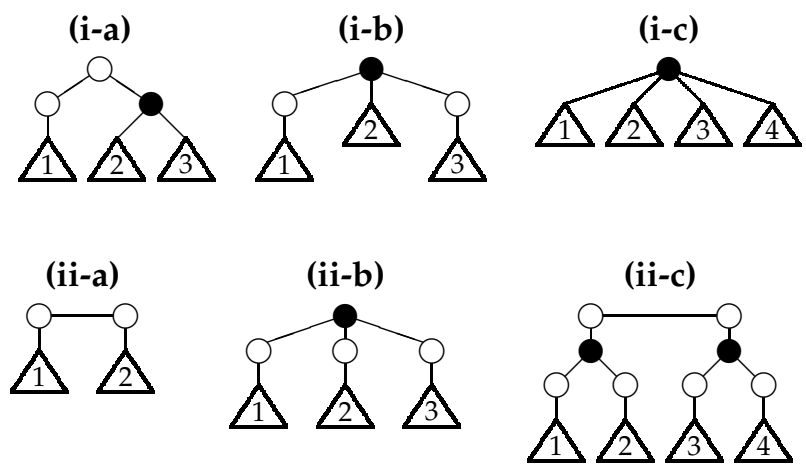

Fig. 3. Each of the subtrees numbered 1-4 is one distinct canonical subtree in these illustrations of different types of topology. In (i-a) subtrees 2 and 3 are co-rooted and separated from subtree 1 . In (i-b) subtrees 1 and 3 are isolated, while subtree 2 is non-isolated. In (i-c) we have a fully co-rooted tagged component tree with the four canonical subtrees. In the second half we have fully separated tagged component trees with (ii-a) two, (ii-b) three, or (ii-c) four canonical subtrees.

that can be indel-saving or indel-neutral depending on whether $u_{1}$ and $u_{2}$ share a tag or not.

\subsubsection{Tag mates}

Let a tagged canonical subtree $\Upsilon(L)$ be separated from a partition subtree $\Upsilon(\mathcal{L})$ by a bad link $P$. As we explain in the following, the bad link $P$ can be possibly covered by an indel-saving out-traversal or by an indel-saving semi-traversal.

First suppose that at least one leaf of the subtree $\Upsilon(\mathcal{L})$ shares a tag with the leaves of $\Upsilon(L)$. In this case, the bad link $P$ can be covered by an indel-saving out-traversal $P^{\prime}$ (with $\tau\left(P^{\prime}\right)=1$ ) and we say that subtree $\Upsilon(\mathcal{L})$ contains a trivial tag mate of $\Upsilon(L)$. An example of trivial A-mate is given in Figure 4 (i).

Now suppose that subtree $\Upsilon(\mathcal{L})$ has no trivial tag mate of $\Upsilon(L)$. In this case $\Upsilon(\mathcal{L})$ might have some internal node that shares a tag with the leaves of $\Upsilon(L)$, therefore it can still be possible to cover the bad link $P$ by an indelsaving semi-traversal. More precisely, let the extended subtree $\Upsilon^{+}(\mathcal{L})$ be $\Upsilon(\mathcal{L} \cup\{p\})$, where $p$ is the bad node of the bad link $P$ that is closest to $\Upsilon(\mathcal{L})$. If the tag set of at least one node $m$ of $\Upsilon^{+}(\mathcal{L})$ shares a tag with the leaves of $\Upsilon(L)$, the node $m$ is said to be a non-trivial tag mate of $\Upsilon(L)$ at $\Upsilon^{+}(\mathcal{L})$. The fact that $\Upsilon^{+}(\mathcal{L})$ contains a nontrivial tag mate of $\Upsilon(L)$ is denoted by $\mathrm{T} \vdash \Upsilon^{+}(\mathcal{L})$, where $\mathrm{T}$ is either the tag $\mathrm{A}$ or the tag B. Examples of non-trivial tag mates are $\mathrm{B} \vdash \Upsilon^{+}\left(L_{\mathrm{A}} \cup L_{\varnothing}\right)$ and $\mathrm{A} \vdash \Upsilon^{+}\left(L_{\varnothing}\right)$, the latter shown in Figure 4 (ii)-(iii).

Observe that, if $\mathcal{L}$ includes the class $L_{\mathrm{AB}}$, it is unnecessary to verify whether the extended subtree $\Upsilon^{+}(\mathcal{L})$ contains a non-trivial tag mate, because in this case it obviously contains a trivial one. 
(i) trivial mate

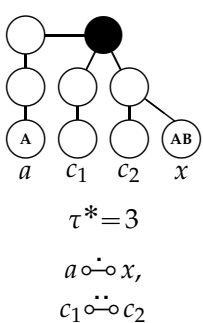

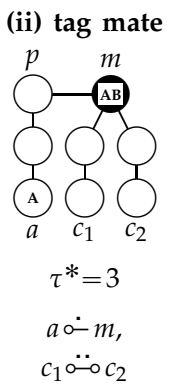

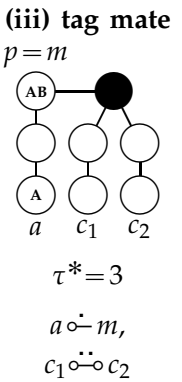

(i) solo leaf

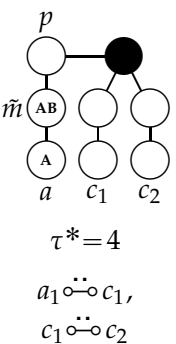

Fig. 4. Tag mates are illustrated in four examples. In (i) the AB-leaf $x$ is a trivial A-mate of $\Upsilon\left(L_{\mathrm{A}}\right)$ at $\Upsilon\left(L_{\varnothing} \cup L_{\mathrm{AB}}\right)$. In examples (ii) and (iii), whose optimal covers have cost 3 , there is no trivial tag mate, but the internal AB-node $m$ is an A-mate of $\Upsilon\left(L_{\mathrm{A}}\right)$ at $\Upsilon\left(L_{\varnothing} \cup\{p\}\right)$. In example (iv) the internal AB-node $\tilde{m}$ is not at $\Upsilon\left(L_{\varnothing} \cup\{p\}\right)$ and an optimal cover has cost 4.

\subsection{Procedure of computing an optimal cover}

For any canonical subtree $\Upsilon(L)$, the cost of an in-traversal of $\Upsilon(L)$ is at most the same as the cost of an outtraversal between $\Upsilon(L)$ and any other canonical subtree. This suggests an approach of searching optimal covers maximizing in-traversals. We need to be careful, however, and also allow out-traversals for covering bad links and possibly unpaired leaves.

Given a tree $T_{\circ}(\alpha, \beta)$ with at least two types of leaves that share no tag, we will search for an optimal cover with a three-step procedure. The first step consists of determining whether the solo leaf exists: it is a leaf of the clean subtree that must be kept by any reduction. The second step consists of safely reducing the sizes of the four subtrees $\Upsilon\left(L_{\mathrm{A}}\right), \Upsilon\left(L_{\mathrm{B}}\right), \Upsilon\left(L_{\varnothing}\right)$ and $\Upsilon\left(L_{\mathrm{AB}}\right)$ of $T_{\circ}(\alpha, \beta)$ with in-traversals, until we reach a certain minimum number of leaves per canonical subtree, obtaining a residual tree. The third step is then finding an optimal cover of the residual tree, that requires a lengthy enumeration of all possible cases.

\subsubsection{Solo leaf}

When reducing the clean subtree, we need to be careful with a particularity of the topology that can influence the cost of an optimal cover. Let the tree $T_{\circ}(\alpha, \beta)$ with at least three leaves have a clean leaf $s$ in a short leaf-branch and let $T_{\circ}^{-s}(\alpha, \beta)$ be the tree obtained by pruning $s$ and performing a flower-contraction in $T_{\circ}(\alpha, \beta)$. Obviously, the tree $T_{\circ}^{-s}(\alpha, \beta)$ has exactly one leaf less than $T_{\circ}(\alpha, \beta)$. If $\tau\left(T_{\circ}(\alpha, \beta)\right)=\tau\left(T_{\circ}^{-s}(\alpha, \beta)\right)+1$, then $s$ is said to be a solo leaf. In other words, the clean leaf $s$ is a solo leaf if there is some optimal cover of $T_{\circ}(\alpha, \beta)$ that includes a short path covering $s$. See examples in Figure 5 (i) and (ii).

The solo leaf is the last leaf to be paired when we search for an optimal cover of $T_{\circ}(\alpha, \beta)$. In some sense, it represents the absence of a "fortress" [1]: if, instead, the last unpaired clean leaf would not be a solo leaf but part of a long leaf-branch, it could only be covered together with its branch by an indel-neutral path, whose cost is 2 .
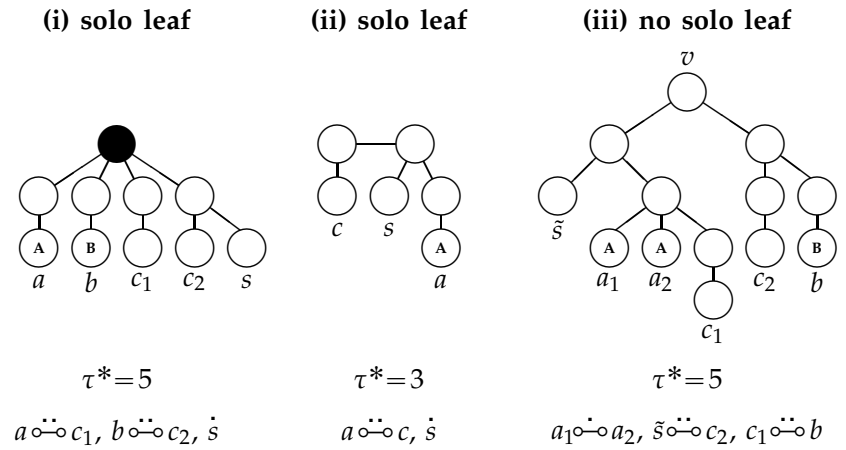

Fig. 5. The concept of solo leaf is illustrated with three examples. In (i) the solo leaf $s$ is in a short leaf-branch of $\Upsilon\left(L_{\varnothing}\right)$. In (ii) the solo leaf $s$ is in a short leaf-branch of $T_{\circ}(\alpha, \beta)$ that is a long leaf-branch of $\Upsilon\left(L_{\varnothing}\right)$. In (iii), although $\tilde{s}$ is in a short leaf-branch of $T_{\circ}(\alpha, \beta)$ and even of $\Upsilon\left(L_{\varnothing}\right)$, no cover including a short path with $\tilde{s}$ is optimal.

In contrast to clean leaves in short leaf-branches of $T_{\circ}(\alpha, \beta)$, tagged leaves in short leaf-branches need no special treatment for the following reasons:

- If originally a tagged canonical subtree is composed of a single leaf, it is indeed important to identify whether its leaf is in a long or in a short leafbranch, since the latter allows it to be covered by a short path. But the single leaf being in a short leafbranch is equivalent to the corresponding subtree being non-isolated, a condition that can be easily verified.

- If a tagged canonical subtree $\Upsilon(L)$ has at least two leaves, there is no advantage of any leaf being in a short leaf-branch: in this case it could possibly be covered by a short path, but if it would be in a long leaf-branch, the whole branch could be covered by an indel-saving path, whose cost is the same as that of a short path.

The difference between keeping a clean leaf or a tagged leaf in a short leaf-branch is illustrated in Figure 6

A tagged component tree $T_{\circ}(\alpha, \beta)$ can have more than one clean short leaf-branch, but in our approach at most one is covered by a short path: even if some optimal cover includes two short paths covering two clean short leaf-branches, there is another optimal cover with the same cost covering the two leaf-branches by an intraversal of $\Upsilon\left(L_{\varnothing}\right)$. Therefore, if the tree has clean short leaf-branches, it suffices to characterize at most one as solo leaf.

However, even if $T_{\circ}(\alpha, \beta)$ has a single clean short leafbranch, the leaf of this branch is not necessarily a solo leaf, as shown in Figure 5 (iii). Actually the procedure of searching for a solo leaf is quite tricky and depends on various aspects of the distinct topologies of a tagged component tree. The only approach we could devise so far tests all possible hypotheses (each clean short leafbranch of $T_{\circ}(\alpha, \beta)$ as the possible solo leaf) and takes one that results in the smallest cover cost. This exhaustive search dominates the complexity of our whole procedure 


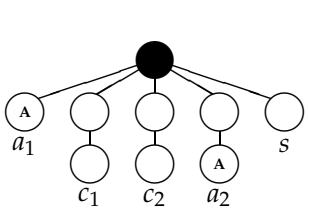

(ii)

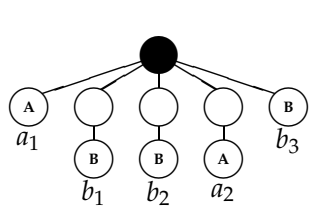

Reductions
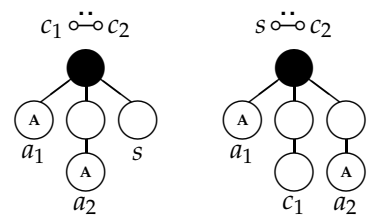

$\tau^{*}=4$ $a_{1} \circ \tau^{*} \circ a_{2}, \dot{s}$

(safe)

$$
\begin{aligned}
& \tau=5 \\
& a_{1} \circ \sim a_{2}, c_{1} \circ a_{2} \\
& \quad \text { (unsafe) }
\end{aligned}
$$

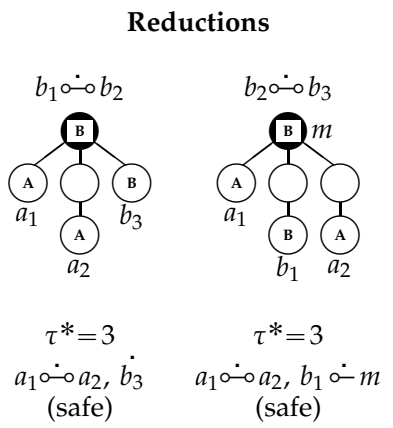

Fig. 6. In (i), subtrees $\Upsilon\left(L_{\mathrm{A}}\right)$ and $\Upsilon\left(L_{\varnothing}\right)$ are co-rooted and the tree has a solo leaf. After the safe $\left(c_{1}, c_{2}\right)$ reduction, the reduced tree keeps the solo leaf and can be covered with cost 2. On the other hand, after the unsafe $\left(s, c_{2}\right)$-reduction, the reduced tree can be covered only with cost 3 . In (ii), subtrees $\Upsilon\left(L_{\mathrm{A}}\right)$ and $\Upsilon\left(L_{\mathrm{B}}\right)$ are co-rooted. After the safe $\left(b_{1}, b_{2}\right)$-reduction, the reduced tree can be covered with cost 2 , including a short path covering leaf $b_{3}$. If instead we do the $\left(b_{2}, b_{3}\right)$-reduction, subtrees $\Upsilon\left(L_{\mathrm{A}}\right)$ and $\Upsilon\left(L_{\mathrm{B}}\right)$ are separated in the reduced tree. However, the flower-contracted vertex $m$ is a tag mate of leaf $b_{1}$. Therefore, this reduction is also safe. In other words, there is no advantage in keeping the short leaf-branch $\left(b_{3}\right)$.

for computing the distance, as we will demonstrate in Section 4.2.4

\subsubsection{Reducing tagged component trees with balanced in-traversals}

Given a canonical subtree $\Upsilon(L)$ with $|L| \geqslant 4$, a balanced in-traversal of $\Upsilon(L)$ is defined as follows:

- If $|L|$ is even, any traversal from the set returned by Algorithm 1 is a balanced in-traversal.

- If $|L|$ is odd, let $P$ be any leaf-branch of $\Upsilon(L)$. A balanced in-traversal of $\Upsilon(L)$ is a balanced intraversal of $\Upsilon(L) \backslash P$.

Let a path $P$ be an in-traversal of some canonical subtree $\Upsilon(L)$. The corresponding $P$-reduction costs 2 if $L=L_{\varnothing}$ and 1 otherwise. It is called a balanced reduction if $P$ is a balanced in-traversal. And it is said to be preserving if $T_{\circ}^{-P}(\alpha, \beta)$ and $T_{\circ}(\alpha, \beta)$ have the same topology with respect to partition subtrees, bad links and solo leaf, and if in $T_{\circ}^{-P}(\alpha, \beta)$ subtree $\Upsilon(L)$ has exactly two leaves less than in $T_{\circ}(\alpha, \beta)$.
Lemma 2: Let $\Upsilon(L)$ be a tagged canonical subtree of $T_{\circ}(\alpha, \beta)$ with $|L| \geqslant 4$. Any balanced $P$-reduction of $\Upsilon(L)$ is preserving.

Proof: Since the in-traversal $P$ is balanced, it "crosses" the subtree $\Upsilon(L)$. This guarantees that the $P$ reduction does not create a new bad leaf, nor a new bad link, nor it increases the length of any remaining leafbranch. Furthermore, the flower-contraction after the reduction preserves all tags, therefore we do not lose any tag mate.

Lemma 3: For any tagged component tree $T_{\circ}(\alpha, \beta)$ with $\left|L_{\varnothing}\right| \geqslant 4$, a preserving $P$-reduction of $\Upsilon\left(L_{\varnothing}\right)$ is any balanced $P$-reduction such that, if $T_{\circ}(\alpha, \beta)$ has a solo leaf $s, P$ does not cover $s$. Such a reduction is called solosafe reduction. If $T_{\circ}(\alpha, \beta)$ has no solo leaf, any balanced reduction of $\Upsilon\left(L_{\varnothing}\right)$ is a solo-safe reduction.

Proof: Here we can repeat the arguments of the proof of Lemma 2 Furthermore, since $P$ does not cover the solo leaf, we do not lose it.

A preserving balanced reduction is very often safe. Indeed, a solo-safe reduction of $\Upsilon\left(L_{\varnothing}\right)$ down to two leaves is always safe. The same is true for any balanced reduction of tagged canonical subtrees $\Upsilon\left(L_{\mathrm{A}}\right)$ and $\Upsilon\left(L_{\mathrm{B}}\right)$ down to two leaves and for any balanced reduction of tagged canonical subtree $\Upsilon\left(L_{\mathrm{AB}}\right)$ down to three leaves. Actually, only in some particular cases a balanced reduction of $\Upsilon\left(L_{\mathrm{AB}}\right)$ from four to two leaves is unsafe. These facts will be demonstrated by the Reduction Bounds stated later in this section. We will also demonstrate that, although a canonical subtree $\Upsilon(L)$ with only three leaves has no balanced in-traversal, a reduction to a single leaf can be safe, even if it is non-preserving (as we showed in Figure 6.

We can now analyse the topologies of each type of tagged component tree and set the lower bounds for safe reductions in each canonical subtree. Since a reduction removes two leaves from the tree, for each case analysis we need to consider whether the number of leaves in the canonical subtree being reduced is even or odd. We start by setting the bounds for canonical subtrees $\Upsilon\left(L_{\mathrm{A}}\right)$ and $\Upsilon\left(L_{\mathrm{B}}\right)$ :

Proposition 2: Each of the canonical subtrees $\Upsilon\left(L_{\mathrm{A}}\right)$ and $\Upsilon\left(L_{B}\right)$ can be safely reduced to one or two leaves in any tagged component tree.

Proof: In Appendix 1.

Let $\ell_{t}$ be the number of leaves in canonical subtree $\Upsilon\left(L_{t}\right)$. The leaf composition of the tree $T_{\circ}(\alpha, \beta)$ is given by the values $\ell_{\mathrm{A}}, \ell_{\mathrm{B}}, \ell_{\varnothing}$ and $\ell_{\mathrm{AB}}$.

Due to the symmetry of costs for A-nodes and for B-nodes in covering paths, we assume without loss of generality that after the reduction we obtain a residual tree in which $\ell_{\mathrm{A}} \geqslant \ell_{\mathrm{B}}$. To achieve this, if $\Upsilon\left(L_{\mathrm{B}}\right)$ is nonempty and if $\Upsilon\left(L_{\mathrm{A}}\right)$ is either empty or has an odd number of leaves, we can simply "swap" A-nodes and Bnodes. Now, for each type of tagged component tree, the bounds of each canonical subtree will be defined in the five Reduction Bounds listed below and whose proofs are given in Appendix 1. 
Reduction Bound 1: Two non-empty canonical subtrees $\Upsilon\left(L_{1}\right)$ and $\Upsilon\left(L_{2}\right)$ such that $L_{1}$ and $L_{2}$ share no tag: Let $\ell_{1}=\left|L_{1}\right|$ and $\ell_{2}=\left|L_{2}\right|$. Without loss of generality, assume that $\Upsilon\left(L_{1}\right)$ is tagged. Given that $w \in\{1,2\}$ is the cost of an in-traversal of $\Upsilon\left(L_{2}\right)$ (that can be tagged or clean), the number of leaves in both subtrees $\Upsilon\left(L_{1}\right)$ and $\Upsilon\left(L_{2}\right)$ can be safely reduced to one or two, with cost $\left\lfloor\left(\ell_{1}-1\right) / 2\right\rfloor+w\left\lfloor\left(\ell_{2}-1\right) / 2\right\rfloor$.

Reduction Bound 2: Three non-empty canonical subtrees $\Upsilon\left(L_{\mathbf{A}}\right), \Upsilon\left(L_{\mathbf{B}}\right)$, and $\Upsilon\left(L_{\varnothing}\right)$ :

1) If $\ell_{\mathrm{A}}$ and $\ell_{\mathrm{B}}$ are odd, and given that $n=\max \left\{\ell_{\varnothing}, 2\right\}$, the number of leaves in subtrees $\Upsilon\left(L_{\mathrm{A}}\right)$ and $\Upsilon\left(L_{\mathrm{B}}\right)$ can be safely reduced to one, and the number of leaves in subtree $\Upsilon\left(L_{\varnothing}\right)$ can be safely reduced to two or three, with cost $\left(\ell_{\mathrm{A}}-1\right) / 2+\left(\ell_{\mathrm{B}}-1\right) / 2+$ $2[(n-2) / 2]$.

2) Otherwise $\ell_{A}$ is even, the number of leaves in subtree $\Upsilon\left(L_{\mathrm{A}}\right)$ can be safely reduced to two and the number of leaves in subtrees $\Upsilon\left(L_{\mathrm{B}}\right)$ and $\Upsilon\left(L_{\varnothing}\right)$ can be safely reduced to one or two, with cost $\left(\ell_{\mathrm{A}}-2\right) / 2+\left[\left(\ell_{\mathrm{B}}-1\right) / 2\right\rfloor+2\left\lfloor\left(\ell_{\varnothing}-1\right) / 2\right\rfloor$.

Reduction Bound 3: Three non-empty canonical subtrees $\Upsilon\left(L_{\mathrm{A}}\right), \Upsilon\left(L_{\mathrm{B}}\right)$, and $\Upsilon\left(L_{\mathrm{AB}}\right)$ :

1) If both $\ell_{A}$ and $\ell_{B}$ are odd, the number of leaves in subtrees $\Upsilon\left(L_{\mathrm{A}}\right)$ and $\Upsilon\left(L_{\mathrm{B}}\right)$ can be safely reduced to one and the number of leaves in subtree $\Upsilon\left(L_{\mathrm{AB}}\right)$ can be safely reduced to one or two, with $\operatorname{cost}\left(\ell_{\mathrm{A}}-\right.$ 1) $/ 2+\left(\ell_{\mathrm{B}}-1\right) / 2+\left\lfloor\left(\ell_{\mathrm{AB}}-1\right) / 2\right\rfloor$.

2) Otherwise, $\ell_{A}$ is even. If $\ell_{B}$ is odd and given that $n=\max \left\{\ell_{\mathrm{AB}}, 2\right\}$, the number of leaves in subtrees $\Upsilon\left(L_{\mathrm{A}}\right)$ can be safely reduced to two, the number of leaves in $\Upsilon\left(L_{B}\right)$ can be safely reduced to one and the number of leaves in subtree $\Upsilon\left(L_{\mathrm{AB}}\right)$ can be safely reduced to two or three, with cost $\left(\ell_{\mathrm{A}}-2\right) / 2+\left(\ell_{\mathrm{B}}-\right.$ $1) / 2+\lfloor(n-2) / 2\rfloor$.

3) Finally, both $\ell_{A}$ and $\ell_{B}$ are even and $n=$ $\max \left\{\ell_{\mathrm{AB}}, 3\right\}$. The number of leaves in subtrees $\Upsilon\left(L_{\mathrm{A}}\right)$ and $\Upsilon\left(L_{\mathrm{B}}\right)$ can be safely reduced to two, and the number of leaves in subtree $\Upsilon\left(L_{\mathrm{AB}}\right)$ can be safely reduced to three or four, with $\operatorname{cost}\left(\ell_{\mathrm{A}}-\right.$ $2) / 2+\left(\ell_{\mathrm{B}}-2\right) / 2+\lfloor(n-3) / 2\rfloor$.

Reduction Bound 4: Three non-empty canonical subtrees $\Upsilon\left(L_{\mathbf{A}}\right), \Upsilon\left(L_{\varnothing}\right)$, and $\Upsilon\left(L_{\mathrm{AB}}\right)$ :

1) If at least one value among $\ell_{A}$ and $\ell_{\varnothing}$ is odd, the number of leaves in each subtree can be safely reduced to one or two, with cost $\left\lfloor\left(\ell_{\mathrm{A}}-1\right) / 2\right\rfloor+$ $2\left\lfloor\left(\ell_{\varnothing}-1\right) / 2\right\rfloor+\left\lfloor\left(\ell_{\mathrm{AB}}-1\right) / 2\right\rfloor$.

2) Otherwise, both $\ell_{A}$ and $\ell_{\varnothing}$ are even and $n=$ $\max \left\{\ell_{\mathrm{AB}}, 2\right\}$. Then the number of leaves in subtrees $\Upsilon\left(L_{\mathrm{A}}\right)$ and $\Upsilon\left(L_{\varnothing}\right)$ can be safely reduced to two, and the number of leaves in subtree $\Upsilon\left(L_{\mathrm{AB}}\right)$ can be safely reduced to two or three, with cost $\left(\ell_{\mathrm{A}}-2\right) / 2+\left(\ell_{\varnothing}-2\right)+\lfloor(n-2) / 2\rfloor$.
Reduction Bound 5: Four non-empty canonical subtrees:

1) If all $\ell_{A}, \ell_{B}$ and $\ell_{\varnothing}$ are odd, the number of leaves in subtrees $\Upsilon\left(L_{\mathrm{A}}\right), \Upsilon\left(L_{\mathrm{B}}\right)$ and $\Upsilon\left(L_{\varnothing}\right)$ can be safely reduced to one, and the number of leaves in subtree $\Upsilon\left(L_{\mathrm{AB}}\right)$ can be safely reduced to one or two, with $\operatorname{cost}\left(\ell_{\mathrm{A}}-1\right) / 2+\left(\ell_{\mathrm{B}}-1\right) / 2+\left(\ell_{\varnothing}-1\right)+\left\lfloor\left(\ell_{\mathrm{AB}}-1\right) / 2\right\rfloor$.

2) Otherwise, if $\ell_{B}$ is odd and among $\ell_{A}$ and $\ell_{\varnothing}$ one value is odd and the other is even, we set $n=\max \left\{\ell_{\mathrm{AB}}, 2\right\}$. The number of leaves in subtree $\Upsilon\left(L_{\mathrm{B}}\right)$ can be safely reduced to one, the number of leaves in subtrees $\Upsilon\left(L_{\mathrm{A}}\right)$ and $\Upsilon\left(L_{\varnothing}\right)$ can be safely reduced to one or two, and the number of leaves in subtree $\Upsilon\left(L_{\mathrm{AB}}\right)$ can be safely reduced to two or three, with cost $\left[\left(\ell_{\mathrm{A}}-1\right) / 2\right\rfloor+\left(\ell_{\mathrm{B}}-1\right) / 2+2\left\lfloor\left(\ell_{\varnothing}-\right.\right.$ $1) / 2\rfloor+\lfloor(n-2) / 2\rfloor$.

3) Finally, if $\ell_{\mathrm{A}}$ is even and at least one value among $\ell_{\mathrm{B}}$ and $\ell_{\varnothing}$ is also even, we set $n=\max \left\{\ell_{\mathrm{AB}}, 3\right\}$. The number of leaves in subtree $\Upsilon\left(L_{\mathrm{A}}\right)$ can be safely reduced to two, the number of leaves in subtrees $\Upsilon\left(L_{\mathrm{B}}\right)$ and $\Upsilon\left(L_{\varnothing}\right)$ can be safely reduced to one or two, and the number of leaves in subtree $\Upsilon\left(L_{\mathrm{AB}}\right)$ can be safely reduced to three or four, with cost $\left(\ell_{\mathrm{A}}-2\right) / 2+\left[\left(\ell_{\mathrm{B}}-1\right) / 2\right\rfloor+2\left\lfloor\left(\ell_{\varnothing}-1\right) / 2\right\rfloor+\lfloor(n-3) / 2\rfloor$.

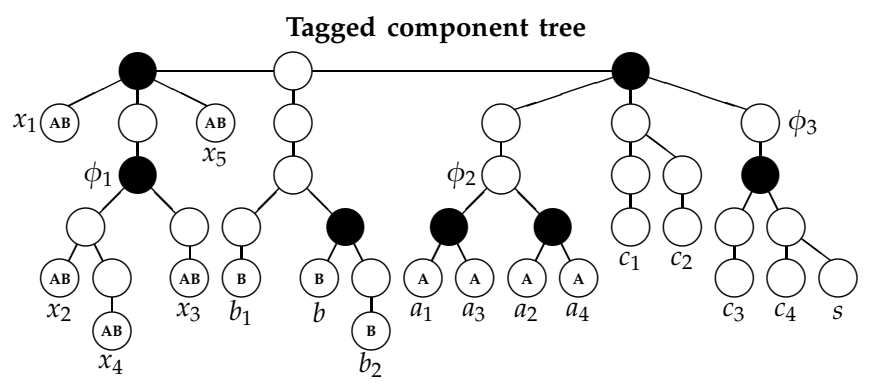

Reduction (cost 7):

$\Upsilon\left(L_{\mathrm{A}}\right): a_{3} \circ \circ a_{4}$

$\Upsilon\left(L_{\mathrm{B}} \backslash\{b\}\right): b_{1} \circ \sim_{\circ} b_{2}$

$\Upsilon\left(L_{\varnothing} \backslash\{s\}\right): c_{1} \circ \ddot{\circ} c_{3}, c_{2} \circ \ddot{\circ} c_{4}$

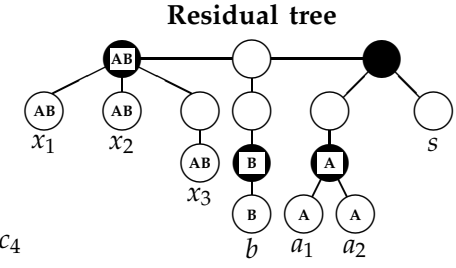

$\Upsilon\left(L_{\mathrm{AB}} \backslash\left\{x_{1}\right\}\right): x_{4} \circ \circ x_{5}$

Fig. 7. Execution of Reduction Bound 5 (case 2) for a tree with $\ell_{\mathrm{A}}=4, \ell_{\mathrm{B}}=3, \ell_{\varnothing}=5$ and $\ell_{\mathrm{AB}}=5$. The leaf $s$ is the solo leaf and vertices $\phi_{1}, \phi_{2}$ and $\phi_{3}$ are, respectively, balanced vertices in subtrees $\Upsilon\left(L_{\mathrm{AB}} \backslash\left\{x_{1}\right\}\right), \Upsilon\left(L_{\mathrm{A}}\right)$ and $\Upsilon\left(L_{\varnothing} \backslash\{s\}\right)$. The obtained residual tree has $\ell_{\mathrm{A}}=2, \ell_{\mathrm{B}}=1$, $\ell_{\varnothing}=1$ and $\ell_{\mathrm{AB}}=3$. Observe that, before and after the reduction, subtrees $\Upsilon\left(L_{\mathrm{A}}\right)$ and $\Upsilon\left(L_{\mathrm{B}}\right)$ are both isolated, while $\Upsilon\left(L_{\varnothing}\right)$ and $\Upsilon\left(L_{\mathrm{AB}}\right)$ are both non-isolated.

Reduction Bounds 1 to 5 jointly define an algorithm to find the residual tree of any tagged component tree (Algorithm 2 in Appendix 1). Once we have the resulting residual tagged component tree (see an example of an 
execution of Reduction Bound 5 - case 2 - in Figure 7), its optimal cover can be obtained from the lengthy enumeration of all possible residual trees, given in the remainder of this section.

\subsubsection{Covering all residual trees}

A residual leaf composition can be represented by a quadruple $\left(\ell_{\mathrm{A}}, \ell_{\mathrm{B}}, \ell_{\varnothing}, \ell_{\mathrm{AB}}\right)$ of four non-negative values that always has $\ell_{\mathrm{A}}$ in the first position, $\ell_{\mathrm{B}}$ in the second position, $\ell_{\varnothing}$ in the third position and $\ell_{\mathrm{AB}}$ in the fourth position. Recall that, due to the symmetry of costs for A-nodes and for B-nodes in covering paths, we assume without loss of generality that $\ell_{\mathrm{A}} \geqslant \ell_{\mathrm{B}}$. According to the results presented so far, we have 56 residual leaf compositions distributed in three groups.

Group 1: Residual trees with exactly two non-empty canonical subtrees $\Upsilon\left(L_{1}\right)$ and $\Upsilon\left(L_{2}\right)$, such that the tag sets of the classes $L_{1}$ and $L_{2}$ share no tag, defined in Reduction Bound 1, This group includes 11 residual leaf compositions listed in Table 2 The complete enumeration is given in Appendix 2. As an example of Group 1, we give the topologies and respective optimal covers of leaf composition $(2,2,0,0)$ in Figure 8 .

TABLE 2

All 11 residual leaf compositions of Group 1

\begin{tabular}{ccc}
\hline \multicolumn{3}{c}{ Reduction Bound 1 } \\
\hline$\left(\ell_{\mathrm{A}}, \ell_{\mathrm{B}}, 0,0\right)$ & $\left(\ell_{\mathrm{A}}, 0, \ell_{\varnothing}, 0\right)$ & $\left(0,0, \ell_{\varnothing}, \ell_{\mathrm{AB}}\right)$ \\
\hline$(1,1,0,0)$ & $(1,0,1,0)$ & $(0,0,1,1)$ \\
& $(1,0,2,0)$ & $(0,0,1,2)$ \\
$(2,1,0,0)$ & $(2,0,1,0)$ & $(0,0,2,1)$ \\
$(2,2,0,0)$ & $(2,0,2,0)$ & $(0,0,2,2)$ \\
\hline
\end{tabular}

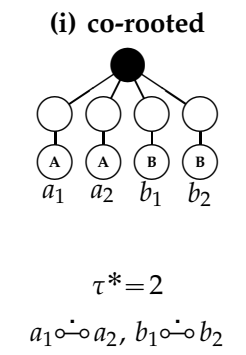

(iii) tag mate

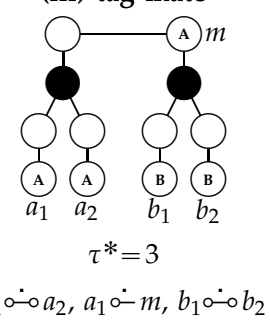

(ii) short link

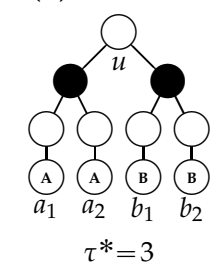

$a_{1} \circ \circ a_{2}, b_{1} \circ \circ b_{2}, \dot{u}$

(iv) long link

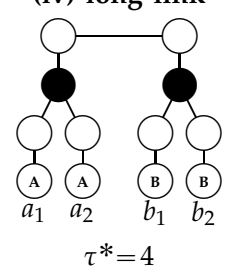

$a_{1} \stackrel{\circ}{\circ} \circ b_{1}, a_{2} \sim \ddot{\circ} b_{2}$
Fig. 8. Possible topologies of leaf composition $(2,2,0,0)$. A symmetric case of (iii) is omitted.
Group 2: Residual trees with exactly three non-empty canonical subtrees, forming three subgroups, defined by Reduction Bounds 2 to 4 . This group includes 25 residual leaf compositions listed in Table 3 . The complete enumeration is given in Appendix 2. In particular, in this group we have the residual leaf composition $(1,1,3,0)$ (Reduction Bound 2, first case) and also residual leaf compositions with three or four leaves in subtree $\Upsilon\left(L_{\mathrm{AB}}\right)$ (cases of Reduction Bounds 3 and 4 ). Some topologies of these leaf compositions can be further reduced by an extra in-traversal and are then said to be reducible. As an example of Group 2, we give the topologies and respective optimal covers of leaf composition $(1,1,3,0)$ and its reduction $(1,1,1,0)$ in Figure 9

TABLE 3

All 25 residual leaf compositions of Group 2

\begin{tabular}{c|cc}
\hline \multicolumn{3}{c}{ Reduction Bound 2 } \\
\hline Case 1 & \multicolumn{2}{c}{ Case 2} \\
$\left(1,1, \ell_{\varnothing}, 0\right)$ & $\left(2,1, \ell_{\varnothing}, 0\right)$ & $\left(2,2, \ell_{\varnothing}, 0\right)$ \\
\hline$(1,1,1,0)$ & $(2,1,1,0)$ & $(2,2,1,0)$ \\
$(1,1,2,0)$ & $(2,1,2,0)$ & $(2,2,2,0)$ \\
$(1,1,3,0)$ & & \\
\hline
\end{tabular}

\begin{tabular}{|c|c|c|}
\hline \multicolumn{3}{|c|}{ Reduction Bound 3} \\
\hline $\begin{array}{c}\text { Case } 1 \\
\left(1,1,0, \ell_{\mathrm{AB}}\right)\end{array}$ & $\begin{array}{c}\text { Case } 2 \\
\left(2,1,0, \ell_{\mathrm{AB}}\right)\end{array}$ & $\begin{array}{c}\text { Case } 3 \\
\left(2,2,0, \ell_{\mathrm{AB}}\right)\end{array}$ \\
\hline $\begin{array}{l}(1,1,0,1) \\
(1,1,0,2)\end{array}$ & $\begin{array}{l}(2,1,0,1) \\
(2,1,0,2) \\
(2,1,0,3)\end{array}$ & $\begin{array}{l}(2,2,0,1) \\
(2,2,0,2) \\
(2,2,0,3) \\
(2,2,0,4)\end{array}$ \\
\hline
\end{tabular}

\begin{tabular}{cccc}
\hline \multicolumn{4}{c}{ Reduction Bound 4} \\
\hline Case 1 & Case 2 \\
$\left(1,0,1, \ell_{\mathrm{AB}}\right)$ & $\left(1,0,2, \ell_{\mathrm{AB}}\right)$ & $\left(2,0,1, \ell_{\mathrm{AB}}\right)$ & $\left(2,0,2, \ell_{\mathrm{AB}}\right)$ \\
\hline$(1,0,1,1)$ & $(1,0,2,1)$ & $(2,0,1,1)$ & $(2,0,2,1)$ \\
$(1,0,1,2)$ & $(1,0,2,2)$ & $(2,0,1,2)$ & $(2,0,2,2)$ \\
& & & $(2,0,2,3)$ \\
\hline
\end{tabular}

Group 3: Residual trees with four non-empty canonical subtrees, defined by Reduction Bound 5, whose cases 2 and 3 include residual leaf compositions with three or four leaves in subtree $\Upsilon\left(L_{\mathrm{AB}}\right)$, containing reducible topologies. In total, this group includes 20 residual leaf compositions listed in Table 4. The complete enumeration is given in Appendix 2. As an example, we consider the leaf composition $(2,2,2,3)$. The non-reducible topologies and respective optimal covers are shown in Figure 12, and the ones reducible to $(2,2,2,1)$ are shown in Figure 13, both in Appendix 2.

\subsubsection{Proof of correctness and complexity}

The correctness of our approach is a consequence of the correctness of Reduction Bounds 1 to 5 and the enumeration of all possible residual trees, as stated in the following theorem. 
Non-reducible topology of leaf composition $(1,1,3,0)$ : both $\Upsilon\left(L_{A}\right)$ and $\Upsilon\left(L_{B}\right)$ isolated, solo leaf, no tag mates

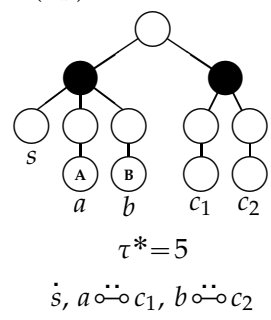

Topologies of leaf composition $(1,1,3,0)$ reducible to $(1,1,1,0)$ (i) $\Upsilon\left(L_{\mathrm{A}}\right)$ non-isolated

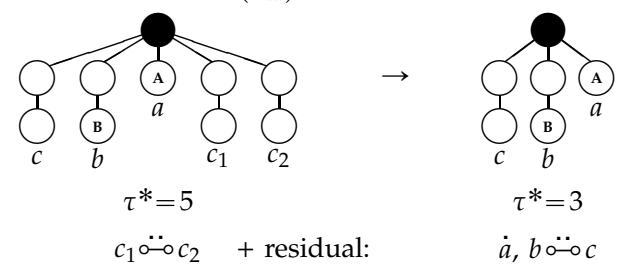

(ii) tag mate

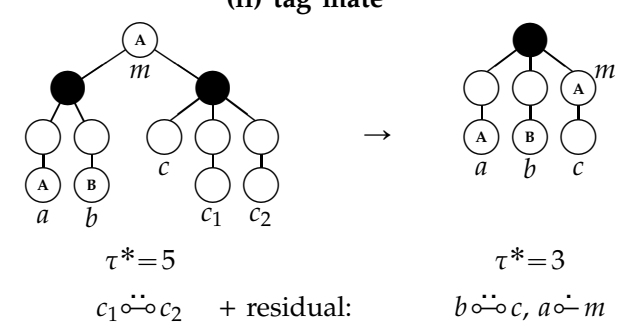

(iii) both $\Upsilon\left(L_{\mathrm{A}}\right)$ and $\Upsilon\left(L_{\mathrm{B}}\right)$ isolated, no solo leaf, no mates

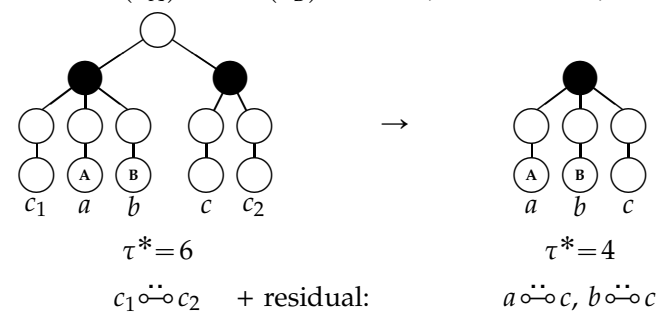

Fig. 9. Leaf composition $(1,1,3,0)$ has a single nonreducible topology (with both $\Upsilon\left(L_{\mathrm{A}}\right)$ and $\Upsilon\left(L_{\mathrm{B}}\right)$ isolated, solo leaf and no tag mates) and three reducible topologies (symmetric cases of (i) and (ii) are omitted).

Theorem 5: Given a tagged component tree $T_{\circ}(\alpha, \beta)$, let $\tau$ be the sum of the cost of applying the appropriate Reduction Bound (among 1,5) to $T_{\circ}(\alpha, \beta)$ and the cost of covering the obtained residual tree $T_{\circ}^{R}(\alpha, \beta)$, that can be found in the full enumeration (in Appendix 2). The value $\tau$ is optimal, that is, $\tau=\tau^{*}(\alpha, \beta)$.

Proof: Reduction Bounds 1 to 5 (whose proofs are given in Appendix 1) give the number of safe balanced in-traversals that we can use to reduce each canonical subtree. For the residual trees, the exhaustive enumeration of all topologies guarantees that we can always find an optimal cover.

The following considerations show that the topology description that we consider contains all necessary properties to the search of optimal covers.

Since the cost of an in-traversal of canonical subtree
TABLE 4

All 20 residual leaf compositions of Group 3

\begin{tabular}{ccc}
\hline \multicolumn{3}{c}{ Reduction Bound 5} \\
\hline Case 1 & \multicolumn{2}{c}{ Case 2} \\
$\left(1,1,1, \ell_{\mathrm{AB}}\right)$ & $\left(1,1,2, \ell_{\mathrm{AB}}\right)$ & $\left(2,1,1, \ell_{\mathrm{AB}}\right)$ \\
\hline$(1,1,1,1)$ & $(1,1,2,1)$ & $(2,1,1,1)$ \\
$(1,1,1,2)$ & $(1,1,2,2)$ & $(2,1,1,2)$ \\
& $(1,1,2,3)$ & $(2,1,1,3)$ \\
\hline \multicolumn{3}{c}{ Case 3 } \\
$\left(2,1,2, \ell_{\mathrm{AB}}\right)$ & $\left(2,2,1, \ell_{\mathrm{AB}}\right)$ & $\left(2,2,2, \ell_{\mathrm{AB}}\right)$ \\
\hline$(2,1,2,1)$ & $(2,2,1,1)$ & $(2,2,2,1)$ \\
$(2,1,2,2)$ & $(2,2,1,2)$ & $(2,2,2,2)$ \\
$(2,1,2,3)$ & $(2,2,1,3)$ & $(2,2,2,3)$ \\
$(2,1,2,4)$ & $(2,2,1,4)$ & $(2,2,2,4)$ \\
\hline
\end{tabular}

$\Upsilon(L)$ is at most the same as the cost of an out-traversal between $\Upsilon(L)$ and any other canonical subtree, in the absence of bad links and if each canonical subtree has an even number of leaves, it is clear that with in-traversals only we cover the tree optimally.

Otherwise, bad links, tag mates and solo leaf are sufficient properties to drive our search for optimal covers:

- Note that, if long bad links exist, one can find an optimal cover by attempting to cover them by only three types of paths: (1) indel-saving out-traversals, (2) indel-saving semi-traversals (with tag mates) and (3) indel-neutral out-traversals. (Another possibility would be to have bridges, that are paths connecting two internal nodes. Indeed, an optimal cover can contain a bridge, but in this case there is always an alternative optimal cover composed of traversals, semi-traversals and at most one short path - see Proposition 5 in Appendix 1.) Therefore, besides the leaves, tag mates are the only nodes we have to consider to cover bad links.

- As explained in Section 4.2.1 if there are unpaired leaves, only in the clean subtree we need to be careful and keep the solo leaf, that is in a short leafbranch of $T_{0}(\alpha, \beta)$, to be the last leaf to be paired. For tagged leaves, it makes no difference whether the leaf is in a short or in a long leaf-branch, unless originally the corresponding subtree is composed of a single leaf (in this case, the leaf being in a short leaf-branch is equivalent to the corresponding subtree being non-isolated, a condition that is considered when we enumerate the covers of all possible residual trees).

With respect to the complexity, the critical point is finding solo-safe reductions of the clean subtree, which results in a quadratic procedure.

Lemma 4: The time and space complexity of our procedure of computing the inversion-indel distance is quadratic in the number of markers $|G|+|A|+|B|$.

Proof: In Appendix 1. 


\section{Conclusion}

In this work we have completed the study of the inversion-indel distance between two chromosomes $\alpha$ and $\beta$ with unequal contents, by presenting an approach to compute the extra cost $\tau^{*}(\alpha, \beta)$ of sorting bad components of the relational diagram $R(\alpha, \beta)$. We gave easy-tocompute lower and upper bounds. For the intermediate instances, our approach is based on the composition of several properties of tagged component trees and the enumeration of optimal covers of a long list of minimal residual trees that can be obtained by reducing them. The whole procedure can be done in quadratic time and space on the number of markers $|G|+|A|+|B|$.

In future work, it seems now possible to address the more general problem of computing the multichromosomal genomic distance [2] with insertions and deletions using techniques similar to those used in this paper combined with the more general component tree presented in [8].

\section{References}

[1] S. Hannenhalli and P. A. Pevzner, "Transforming cabbage into turnip: polynomial algorithm for sorting signed permutations by reversals," Journal of the ACM, vol. 46, no. 1, pp. 1-27, 1999, (A preliminary version appeared in the Proceedings of STOC 1995.).

[2] - "Transforming men into mice (polynomial algorithm for genomic distance problem)," in Proceedings of the 36th Annual Symposium of the Foundations of Computer Science (FOCS 1995). IEEE Press, 1995, pp. 581-592.

[3] N. El-Mabrouk, "Genome rearrangement by reversals and insertions/deletions of contiguous segments," in Proceedings of the 11th Annual Symposium on Combinatorial Pattern Matching (CPM 2000), ser. LNCS, vol. 1848. Springer Verlag, 2000, pp. 222-234, (An extended version is available here: http://www.iro.umontreal.ca/ mabrouk/Publications/jda.ps ).

[4] S. Yancopoulos, O. Attie, and R. Friedberg, "Efficient sorting of genomic permutations by translocation, inversion and block interchange," Bioinformatics, vol. 21, no. 16, pp. 3340-3346, 2005.

[5] A. Bergeron, J. Mixtacki, and J. Stoye, "A unifying view of genome rearrangements," in Proceedings of the 6th International Conference on Algorithms in Bioinformatics (WABI 2006), ser. LNBI, vol. 4175. Springer Verlag, 2006, pp. 163-173.

[6] S. Yancopoulos and R. Friedberg, "DCJ path formulation for genome transformations which include insertions, deletions, and duplications," Journal of Computational Biology, vol. 16, no. 10, pp. 1311-1338, 2009, (A preliminary version appeared in the Proceedings of RECOMB-CG 2008.).

[7] M. D. V. Braga, E. Willing, and J. Stoye, "Double cut and join with insertions and deletions," Journal of Computational Biology, vol. 18, no. 9, pp. 1167-1184, 2011, (A preliminary version appeared in the Proceedings of WABI 2010.).

[8] A. Bergeron, J. Mixtacki, and J. Stoye, "A new linear time algorithm to compute the genomic distance via the double cut and join distance," Theoretical Computer Science, vol. 410, no. 51, pp. 53005316, 2009, (A preliminary version appeared in the Proceedings of CPM 2008.).

[9] E. Willing, S. Zaccaria, M. D. V. Braga, and J. Stoye, "On the inversion-indel distance," BMC Bioinformatics, vol. 14, no. Suppl. 15, p. S3, 2013, (Proceedings of RECOMB-CG 2013.).

[10] M. D. V. Braga, "An overview of genomic distances modeled with indels," in Proceedings of the Conference on Computability in Europe (CiE 2013), ser. LNCS, vol. 7921. Springer Verlag, 2013, pp. 22-31.

[11] R. Friedberg, A. E. Darling, and S. Yancopoulos, "Genome rearrangement by the double cut and join operation," in Bioinformatics, Volume I: Data, Sequence Analysis, and Evolution, ser. Methods in Molecular Biology, J. M. Keith, Ed. Humana Press, 2008, vol. 452, pp. 385-416.
[12] J. Meidanis, M. E. M. T. Walter, and Z. Dias, "Reversal distance of signed circular chromosomes," Institute of Computing, University of Campinas, Campinas, SP, Brazil, Relatório Técnico IC-00-23, December 2000

[13] A. Bergeron, J. Mixtacki, and J. Stoye, "The inversion distance problem," in Mathematics of Evolution and Phylogeny, O. Gascuel, Ed. Oxford University Press, 2005, ch. 10, pp. 262-290, (A preliminary version appeared in the Proceedings of CPM 2004.).

[14] P. L. Erdös, L. Soukup, and J. Stoye, "Balanced vertices in trees and a simpler algorithm to compute the genomic distance," Applied Mathematics Letters, vol. 24, no. 1, pp. 82-86, 2011.

[15] A. Gyárfás and J. Lehel, "A helly-type problem in trees," in Colloquia Mathematica Scocietatis János Bólyai, ser. Combinatorial Theory and its Applications II, vol. 4, 1970, pp. 571-584. 


\section{Appendix 1: Omitted Proofs and Algorithms}

\section{Proofs}

The bounds given by Proposition 2 and Reduction Bounds 1 to 5 were set assuming the worst case of fully separated trees. The reason is that, if a fully separated tree $T_{1}$ and non fully separated tree $T_{2}$ have the same number of leaves per canonical subtree, in $T_{1}$ we might need to keep more leaves for covering bad links. In other words, if, together with the reduction, some amount of remaining leaves is enough for giving an optimal cover of $T_{1}$, the same number is clearly enough for giving an optimal cover of $T_{2}$.

Proposition 2. Each of the canonical subtrees $\Upsilon\left(L_{\mathrm{A}}\right)$ and $\Upsilon\left(L_{\mathrm{B}}\right)$ can be safely reduced to one or two leaves in any tagged component tree.

Proof: Without loss of generality, we will do the case analsis considering only $\Upsilon\left(L_{\mathrm{A}}\right)$. First suppose we have a tagged component tree with two non-empty canonical subtrees $\Upsilon\left(L_{\mathrm{A}}\right)$ and $\Upsilon(L)$. Then suppose $\Upsilon\left(L_{\mathrm{A}}\right)$ has three leaves and all of them are endpoints of out-traversals to canonical subtree $\Upsilon(L)$. In this case we could replace two out-traversals between $\Upsilon\left(L_{\mathrm{A}}\right)$ and $\Upsilon(L)$ by an in-traversal of $\Upsilon\left(L_{\mathrm{A}}\right)$ and an in-traversal of $\Upsilon(L)$. Note that $L$ can be either $L_{\mathrm{B}}$ or $L_{\varnothing}$, but in both cases this replacement would lead to a smaller cost than the original traversals. Now suppose we have a tagged component tree with three non-empty canonical subtrees $\Upsilon\left(L_{\mathrm{A}}\right), \Upsilon\left(L_{1}\right)$ and $\Upsilon\left(L_{2}\right)$. Then suppose $\Upsilon\left(L_{\mathrm{A}}\right)$ has three leaves and two of them are endpoints of out-traversals to canonical subtree $\Upsilon\left(L_{1}\right)$, while the third leaf of $\Upsilon\left(L_{\mathrm{A}}\right)$ is endpoint of an out-traversal to canonical subtree $\Upsilon\left(L_{2}\right)$. In this case we could replace an out-traversal between $\Upsilon\left(L_{\mathrm{A}}\right)$ and $\Upsilon\left(L_{1}\right)$ and the out-traversal between $\Upsilon\left(L_{\mathrm{A}}\right)$ and $\Upsilon\left(L_{2}\right)$ by an in-traversal of $\Upsilon\left(L_{\mathrm{A}}\right)$ and an out-traversal between $\Upsilon\left(L_{1}\right)$ and $\Upsilon\left(L_{2}\right)$. By assigning the possible canonical subtrees to $L_{1}$ and $L_{2}$, one can verify that this replacement would lead to at most the same cost as the original traversals. (A similar analysis can be done for the case in which the tagged component tree has four non-empty canonical subtrees.) Clearly, if a reduction from 3 to 1 is possible, then a reduction from 4 to 2 is also possible.

Reduction Bound 1) Two non-empty canonical subtrees $\Upsilon\left(L_{1}\right)$ and $\Upsilon\left(L_{2}\right)$ such that $L_{1}$ and $L_{2}$ share no tag: Let $\ell_{1}=\left|L_{1}\right|$ and $\ell_{2}=\left|L_{2}\right|$. Without loss of generality, assume that $\Upsilon\left(L_{1}\right)$ is tagged. Given that $w \in\{1,2\}$ is the cost of an in-traversal of $\Upsilon\left(L_{2}\right)$ (that can be tagged or clean), the number of leaves in both subtrees $\Upsilon\left(L_{1}\right)$ and $\Upsilon\left(L_{2}\right)$ can be safely reduced to one or two, with cost $\left\lfloor\left(\ell_{1}-1\right) / 2\right\rfloor+w\left\lfloor\left(\ell_{2}-1\right) / 2\right\rfloor$.

Proof: Two leaves from each canonical subtree are sufficient to cover the possible bad link between them and compensate an unpaired leaf from the other subtree. It is then clear that, if $\ell_{1} \geqslant 4$ (respect. $\ell_{2} \geqslant 4$ ), it can be reduced to 2 or 3 by preserving reductions (Lemma 2) that are safe. The question now is whether $\ell_{1}$ and/or $\ell_{2}$ can still be reduced from three to one. If one subtree has only one leaf, it is clear that we can reduce the other from three leaves to one. If both subtrees have three leaves, it is clear that both can be reduced to one leaf. If one subtree has three leaves and the other has two, we have the following possibilities:

(i) $L_{1}$ is either $L_{\mathrm{A}}$ or $L_{\mathrm{AB}}$ and $L_{2}=L_{\varnothing}$ : in this case we have one out-traversal to cover the bad link. Suppose we have a cover that contains another out-traversal. This cover would have a cost of five. However, if instead we do an in-traversal in each canonical subtree, we also have a cost of five, therefore this reduction is safe.

(ii) $L_{1}=L_{\mathrm{A}}$ and $L_{2}=L_{\mathrm{B}}$ : the reduction in this case was proven to be safe by Proposition 2

The following proposition will be used to prove the correctness of Reduction Bounds 2 to 4

Proposition 3: The bad links of a fully separated tagged component tree $T_{\circ}(\alpha, \beta)$ with three non-empty canonical subtrees $\Upsilon\left(L_{1}\right), \Upsilon\left(L_{2}\right)$ and $\Upsilon\left(L_{3}\right)$ can be covered by two paths, one connecting $\Upsilon\left(L_{1}\right)$ and $\Upsilon\left(L_{2}\right)$, and the other connecting $\Upsilon\left(L_{1}\right)$ and $\Upsilon\left(L_{3}\right)$.

Proof: We simply refer to Figure 3 (ii-b) of the paper. It is clear that if we pick any of the three subtrees, say subtree no. 1, a pair with a path between subtrees 1 and 2 and a path between subtrees 1 and 3 covers all bad links of the tagged component tree. 
Reduction Bound 2, Three non-empty canonical subtrees $\Upsilon\left(\boldsymbol{L}_{\mathbf{A}}\right), \Upsilon\left(\boldsymbol{L}_{\mathbf{B}}\right)$, and $\Upsilon\left(\boldsymbol{L}_{\varnothing}\right)$ :

1) If $\ell_{A}$ and $\ell_{B}$ are odd, and given that $n=\max \left\{\ell_{\varnothing}, 2\right\}$, the number of leaves in subtrees $\Upsilon\left(L_{A}\right)$ and $\Upsilon\left(L_{B}\right)$ can be safely reduced to one, and the number of leaves in subtree $\Upsilon\left(L_{\varnothing}\right)$ can be safely reduced to two or three, with cost $\left(\ell_{\mathrm{A}}-1\right) / 2+\left(\ell_{\mathrm{B}}-1\right) / 2+2\lfloor(n-2) / 2\rfloor$.

2) Otherwise $\ell_{\mathrm{A}}$ is even, the number of leaves in subtree $\Upsilon\left(L_{\mathrm{A}}\right)$ can be safely reduced to two and the number of leaves in subtrees $\Upsilon\left(L_{B}\right)$ and $\Upsilon\left(L_{\varnothing}\right)$ can be safely reduced to one or two, with cost $\left(\ell_{A}-2\right) / 2+\left\lfloor\left(\ell_{B}-1\right) / 2\right\rfloor+$ $2\left\lfloor\left(\ell_{\varnothing}-1\right) / 2\right\rfloor$

Proof: Proposition 2 guarantees that $\Upsilon\left(L_{\mathrm{A}}\right)$ and $\Upsilon\left(L_{\mathrm{B}}\right)$ can be safely reduced to one or two leaves. Since all bad links can be covered by two out-traversals (Proposition 3), we need to reserve two leaves from $\Upsilon\left(L_{\varnothing}\right)$ to cover the bad links, and two other leaves from $\Upsilon\left(L_{\varnothing}\right)$ to possibly compensate unpaired leaves in the other two subtrees, meaning that the number of leaves in $\Upsilon\left(L_{\varnothing}\right)$ can be safely reduced to four or five. Further reductions depend on the following case analysis.

Case 1: Note that, if $\ell_{\mathrm{A}}$ is odd and $\ell_{\mathrm{B}}$ is odd, they can be both reduced to one and we do not need to compensate unpaired leaves. Then the number of leaves in $\Upsilon\left(L_{\varnothing}\right)$ can be safely reduced to two or three. Let us examine whether we could still reduce from three to one: if both tagged subtrees are isolated and the tree has the solo leaf, such a reduction could either create a new leaf or transform the solo leaf into a leaf in a long leaf-branch, being then unsafe. Therefore in this case the bounds for the clean subtree are two or three.

Case 2: Now let $\ell_{\mathrm{A}}$ be even. Suppose that we have five leaves in $\Upsilon\left(L_{\varnothing}\right)$, two leaves in $\Upsilon\left(L_{\mathrm{B}}\right)$ and four outtraversals involving $\Upsilon\left(L_{\varnothing}\right)$. We could then replace, at the same cost, two of these out-traversals by an in-traversal of $\Upsilon\left(L_{\varnothing}\right)$ and an out-traversal between $\Upsilon\left(L_{\mathrm{A}}\right)$ and $\Upsilon\left(L_{\mathrm{B}}\right)$. But then we would have a triangle covering the bad links, therefore we could replace one other out-traversal between $\Upsilon\left(L_{\varnothing}\right)$ and $\Upsilon\left(L_{A}\right)$ by an in-traversal of $\Upsilon\left(L_{\varnothing}\right)$ (covering its unpaired leaf) and an in-traversal of $\Upsilon\left(L_{\mathrm{A}}\right)$, at the same cost. (A similar analysis could be done for the case of a single leaf in $\Upsilon\left(L_{B}\right)$.) Consequently, if the number of leaves in $\Upsilon\left(L_{\varnothing}\right)$ is odd, it can be safely reduced to one. Clearly, if the number of leaves in $\Upsilon\left(L_{\varnothing}\right)$ is even, it can be safely reduced to two.

Reduction Bound 3, Three non-empty canonical subtrees $\Upsilon\left(\boldsymbol{L}_{\mathbf{A}}\right), \Upsilon\left(\boldsymbol{L}_{\mathbf{B}}\right)$, and $\Upsilon\left(\boldsymbol{L}_{\mathrm{AB}}\right)$ :

1) If both $\ell_{A}$ and $\ell_{B}$ are odd, the number of leaves in subtrees $\Upsilon\left(L_{A}\right)$ and $\Upsilon\left(L_{B}\right)$ can be safely reduced to one and the number of leaves in subtree $\Upsilon\left(L_{\mathrm{AB}}\right)$ can be safely reduced to one or two, with cost $\left(\ell_{\mathrm{A}}-1\right) / 2+\left(\ell_{\mathrm{B}}-\right.$ 1) $/ 2+\left\lfloor\left(\ell_{\mathrm{AB}}-1\right) / 2\right\rfloor$.

2) Otherwise, $\ell_{A}$ is even. If $\ell_{B}$ is odd and given that $n=\max \left\{\ell_{A B}, 2\right\}$, the number of leaves in subtrees $\Upsilon\left(L_{A}\right)$ can be safely reduced to two, the number of leaves in $\Upsilon\left(L_{B}\right)$ can be safely reduced to one and the number of leaves in subtree $\Upsilon\left(L_{\mathrm{AB}}\right)$ can be safely reduced to two or three, with cost $\left(\ell_{\mathrm{A}}-2\right) / 2+\left(\ell_{\mathrm{B}}-1\right) / 2+\lfloor(n-2) / 2\rfloor$.

3) Finally, both $\ell_{\mathrm{A}}$ and $\ell_{\mathrm{B}}$ are even and $n=\max \left\{\ell_{\mathrm{AB}}, 3\right\}$. The number of leaves in subtrees $\Upsilon\left(L_{\mathrm{A}}\right)$ and $\Upsilon\left(L_{\mathrm{B}}\right)$ can be safely reduced to two, and the number of leaves in subtree $\Upsilon\left(L_{\mathrm{AB}}\right)$ can be safely reduced to three or four, with cost $\left(\ell_{\mathrm{A}}-2\right) / 2+\left(\ell_{\mathrm{B}}-2\right) / 2+\lfloor(n-3) / 2\rfloor$.

Proof: Proposition 2 guarantees that $\Upsilon\left(L_{\mathrm{A}}\right)$ and $\Upsilon\left(L_{\mathrm{B}}\right)$ can be safely reduced to one or two leaves. Since all bad links can be covered by two out-traversals (Proposition 3 ), we need to reserve two leaves from $\Upsilon\left(L_{A B}\right)$ to cover the bad links and two other leaves from $\Upsilon\left(L_{\mathrm{AB}}\right)$ to possibly compensate unpaired leaves in the other two subtrees, meaning that the number of leaves in $\Upsilon\left(L_{\mathrm{AB}}\right)$ can be safely reduced to four or five. Further reductions depend on the following case analysis.

Case 1: Note that, if $\ell_{\mathrm{A}}$ is odd and $\ell_{\mathrm{B}}$ is odd, they can be both reduced to one and we do not need to compensate unpaired leaves. Then number of leaves in $\Upsilon\left(L_{\mathrm{AB}}\right)$ can be safely reduced to two or three. But actually we could still reduce from three to one: suppose the unpaired (third) leaf of subtree $\Upsilon\left(L_{\mathrm{AB}}\right)$ is covered by an out-traversal to one of the other two subtrees. At the same cost we could replace it by an in-traversal of $\Upsilon\left(L_{\mathrm{AB}}\right)$. Therefore in this case $\Upsilon\left(L_{\mathrm{AB}}\right)$ can be safely reduced to one or two.

Case 2: Now let $\ell_{\mathrm{A}}$ be even, that is reduced to two, and $\ell_{\mathrm{B}}$ be odd, that is reduced to one. The number of leaves in $\Upsilon\left(L_{\mathrm{AB}}\right)$ can be then safely reduced to three or four. But actually we could still reduce from four to two: suppose 
the unpaired (fourth) leaf of subtree $\Upsilon\left(L_{\mathrm{AB}}\right)$ is covered by an out-traversal to one of the other two subtrees. At the same cost we could replace it by an in-traversal of $\Upsilon\left(L_{\mathrm{AB}}\right)$. Therefore in this case $\Upsilon\left(L_{\mathrm{AB}}\right)$ can be safely reduced to two or three.

Case 3: Finally, if $\ell_{\mathrm{A}}$ is even and $\ell_{\mathrm{B}}$ is even, they can be both reduced to two. The number of leaves in $\Upsilon\left(L_{\mathrm{AB}}\right)$ can be then safely reduced to four or five. But actually we could still reduce from five to three: suppose the unpaired (fifth) leaf of subtree $\Upsilon\left(L_{\mathrm{AB}}\right)$ is covered by an out-traversal to one of the other two subtrees. At the same cost we could replace it by an in-traversal of $\Upsilon\left(L_{\mathrm{AB}}\right)$. Therefore in this case $\Upsilon\left(L_{\mathrm{AB}}\right)$ can be safely reduced to three or four.

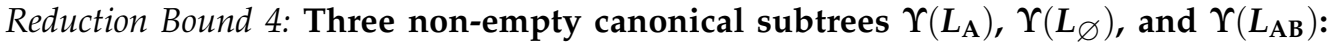

1) If at least one value among $\ell_{\mathrm{A}}$ and $\ell_{\varnothing}$ is odd, the number of leaves in each subtree can be safely reduced to one or two, with cost $\left\lfloor\left(\ell_{\mathrm{A}}-1\right) / 2\right\rfloor+2\left\lfloor\left(\ell_{\varnothing}-1\right) / 2\right\rfloor+\left\lfloor\left(\ell_{\mathrm{AB}}-1\right) / 2\right\rfloor$.

2) Otherwise, both $\ell_{\mathrm{A}}$ and $\ell_{\varnothing}$ are even and $n=\max \left\{\ell_{\mathrm{AB}}, 2\right\}$. Then the number of leaves in subtrees $\Upsilon\left(L_{\mathrm{A}}\right)$ and $\Upsilon\left(L_{\varnothing}\right)$ can be safely reduced to two, and the number of leaves in subtree $\Upsilon\left(L_{\mathrm{AB}}\right)$ can be safely reduced to two or three, with cost $\left(\ell_{\mathrm{A}}-2\right) / 2+\left(\ell_{\varnothing}-2\right)+\lfloor(n-2) / 2\rfloor$.

Proof: Proposition 2 guarantees that $\Upsilon\left(L_{\mathrm{A}}\right)$ can be safely reduced to one or two leaves. We also know that all bad links can be covered by two out-traversals (Proposition 3) and we can always pair $\Upsilon\left(L_{\mathrm{AB}}\right)$ with $\Upsilon\left(L_{\mathrm{A}}\right)$ and $\Upsilon\left(L_{\varnothing}\right)$ with one of the other two subtrees. Note that $\Upsilon\left(L_{\mathrm{AB}}\right)$ is a more "powerful" version of $\Upsilon\left(L_{\mathrm{B}}\right)$, therefore in the following analysis we can often generalize the statements of Reduction Bound 2.

Case 1: Suppose $\ell_{\mathrm{A}}$ is odd, being reduced to one.

- If $\ell_{\varnothing}$ is odd and $\ell_{\mathrm{AB}}$ is odd, by generalizing Reduction Bound 2 - Case 1, we know that $\Upsilon\left(L_{\mathrm{AB}}\right)$ can be safely reduced to one and $\Upsilon\left(L_{\varnothing}\right)$ can be safely reduced to three. But, actually, here $\Upsilon\left(L_{\varnothing}\right)$ can also be reduced from three to one: one leaf is enough to cover the eventual bad link separating $\Upsilon\left(L_{\varnothing}\right)$ from $\bar{\Upsilon}\left(L_{\varnothing}\right)$.

- If $\ell_{\varnothing}$ is odd and $\ell_{\mathrm{AB}}$ is even, by generalizing Reduction Bound 2 - Case 2, we know that $\Upsilon\left(L_{\mathrm{AB}}\right)$ can be safely reduced to two and $\Upsilon\left(L_{\varnothing}\right)$ can be safely reduced to one.

- If $\ell_{\varnothing}$ is even and $\ell_{\mathrm{AB}}$ is odd, by generalizing Reduction Bound 2 - Case 2, we know that $\Upsilon\left(L_{\mathrm{AB}}\right)$ can be safely reduced to one and $\Upsilon\left(L_{\varnothing}\right)$ can be safely reduced to two.

Case 2: Suppose $\ell_{\mathrm{A}}$ is even, being reduced to two.

- If $\ell_{\varnothing}$ is odd, by generalizing Reduction Bound 2 - Case 2, we know that $\Upsilon\left(L_{\varnothing}\right)$ can be safely reduced to one and the number of leaves in $\Upsilon\left(L_{\mathrm{AB}}\right)$ can be safely reduced to one or two.

- If $\ell_{\varnothing}$ is even, by generalizing Reduction Bound 2 - Case 2, we know that $\Upsilon\left(L_{\varnothing}\right)$ can be safely reduced to two. Then the number of leaves in $\Upsilon\left(L_{\mathrm{AB}}\right)$ can be safely reduced to three or four. But actually we can still reduce it from four to two: suppose we have two out-traversals between subtrees $\Upsilon\left(L_{\mathrm{AB}}\right)$ and $\Upsilon\left(L_{\mathrm{A}}\right)$ and two out-traversals between subtrees $\Upsilon\left(L_{\mathrm{AB}}\right)$ and $\Upsilon\left(L_{\varnothing}\right)$. We could then replace one out-traversal between $\Upsilon\left(L_{\mathrm{AB}}\right)$ and $\Upsilon\left(L_{\mathrm{A}}\right)$ and one out-traversal between $\Upsilon\left(L_{\mathrm{AB}}\right)$ and $\Upsilon\left(L_{\varnothing}\right)$ by one out-traversal between $\Upsilon\left(L_{\mathrm{A}}\right)$ and $\Upsilon\left(L_{\varnothing}\right)$ and one in-traversal of $\Upsilon\left(L_{\mathrm{AB}}\right)$. Therefore in this case $\Upsilon\left(L_{\mathrm{AB}}\right)$ can be safely reduced to two.

The following proposition will be used to prove the correctness of Reduction Bound 5

Proposition 4: The bad links of a fully separated tagged component tree $T_{\circ}(\alpha, \beta)$ with four non-empty canonical subtrees $\Upsilon\left(L_{1}\right), \Upsilon\left(L_{2}\right), \Upsilon\left(L_{3}\right)$ and $\Upsilon\left(L_{4}\right)$, such that $\Upsilon\left(L_{1} \cup L_{2}\right)$ is separated from $\Upsilon\left(L_{3} \cup L_{4}\right)$ can be covered by two "crossing" paths, that either connect $\Upsilon\left(L_{1}\right)$ to $\Upsilon\left(L_{3}\right)$ and $\Upsilon\left(L_{2}\right)$ to $\Upsilon\left(L_{4}\right)$ or connect $\Upsilon\left(L_{1}\right)$ to $\Upsilon\left(L_{4}\right)$ and $\Upsilon\left(L_{2}\right)$ to $\Upsilon\left(L_{3}\right)$.

Proof: We simply refer to Figure 3 (ii-c) of the paper. Note that partition subtrees $\Upsilon(1 \cup 3)$ and $\Upsilon(2 \cup 4)$ are co-rooted. It is then clear that a pair with a path between subtrees 1 and 3 and a path between subtrees 2 and 4 covers all bad links of the tagged component tree. A second choice also exists, because $\Upsilon(1 \cup 4)$ and $\Upsilon(2 \cup 3)$ are 
also co-rooted. Therefore a pair with a path between subtrees 1 and 4 and a path between subtrees 2 and 3 also covers all bad links of the tagged component tree.

\section{Reduction Bound 5 . Four non-empty canonical subtrees:}

1) If all $\ell_{\mathrm{A}}, \ell_{\mathrm{B}}$ and $\ell_{\varnothing}$ are odd, the number of leaves in subtrees $\Upsilon\left(L_{\mathrm{A}}\right), \Upsilon\left(L_{\mathrm{B}}\right)$ and $\Upsilon\left(L_{\varnothing}\right)$ can be safely reduced to one, and the number of leaves in subtree $\Upsilon\left(L_{\mathrm{AB}}\right)$ can be safely reduced to one or two, with cost $\left(\ell_{\mathrm{A}}-1\right) / 2+$ $\left(\ell_{\mathrm{B}}-1\right) / 2+\left(\ell_{\varnothing}-1\right)+\left\lfloor\left(\ell_{\mathrm{AB}}-1\right) / 2\right\rfloor$.

2) Otherwise, if $\ell_{\mathrm{B}}$ is odd and among $\ell_{\mathrm{A}}$ and $\ell_{\varnothing}$ one value is odd and the other is even, we set $n=\max \left\{\ell_{\mathrm{AB}}, 2\right\}$. The number of leaves in subtree $\Upsilon\left(L_{\mathrm{B}}\right)$ can be safely reduced to one, the number of leaves in subtrees $\Upsilon\left(L_{\mathrm{A}}\right)$ and $\Upsilon\left(L_{\varnothing}\right)$ can be safely reduced to one or two, and the number of leaves in subtree $\Upsilon\left(L_{\mathrm{AB}}\right)$ can be safely reduced to two or three, with cost $\left\lfloor\left(\ell_{\mathrm{A}}-1\right) / 2\right\rfloor+\left(\ell_{\mathrm{B}}-1\right) / 2+2\left\lfloor\left(\ell_{\varnothing}-1\right) / 2\right\rfloor+\lfloor(n-2) / 2\rfloor$.

3) Finally, if $\ell_{\mathrm{A}}$ is even and at least one value among $\ell_{\mathrm{B}}$ and $\ell_{\varnothing}$ is also even, we set $n=\max \left\{\ell_{\mathrm{AB}}, 3\right\}$. The number of leaves in subtree $\Upsilon\left(L_{\mathrm{A}}\right)$ can be safely reduced to two, the number of leaves in subtrees $\Upsilon\left(L_{\mathrm{B}}\right)$ and $\Upsilon\left(L_{\varnothing}\right)$ can be safely reduced to one or two, and the number of leaves in subtree $\Upsilon\left(L_{\mathrm{AB}}\right)$ can be safely reduced to three or four, with cost $\left(\ell_{\mathrm{A}}-2\right) / 2+\left\lfloor\left(\ell_{\mathrm{B}}-1\right) / 2\right\rfloor+2\left\lfloor\left(\ell_{\varnothing}-1\right) / 2\right\rfloor+\lfloor(n-3) / 2\rfloor$.

Proof: Proposition 2 guarantees that $\Upsilon\left(L_{\mathrm{A}}\right)$ and $\Upsilon\left(L_{\mathrm{B}}\right)$ can be safely reduced to one or two leaves. Since a tree with four canonical subtrees is a generalization of case 2 of Reduction Bound 2 , we also know that $\Upsilon\left(L_{\varnothing}\right)$ can be safely reduced to one or two leaves. Furthermore, all bad links can be covered by two out-traversals (Proposition 4 ). Therefore we can cover the eventual bad links by pairing $\Upsilon\left(L_{\varnothing}\right)$ with either $\Upsilon\left(L_{\mathrm{A}}\right)$ or $\Upsilon\left(L_{\mathrm{B}}\right)$ and $\Upsilon\left(L_{\mathrm{AB}}\right)$ with either $\Upsilon\left(L_{\mathrm{A}}\right)$ or $\Upsilon\left(L_{\mathrm{B}}\right)$. We now need to examine the bounds for the subtree $\Upsilon\left(L_{\mathrm{AB}}\right)$.

Case 1: Note that, if $\ell_{\mathrm{A}}$ is odd, $\ell_{\mathrm{B}}$ is odd and $\ell_{\varnothing}$ is odd, they can all be reduced to one. Consequently, the number of leaves in $\Upsilon\left(L_{\mathrm{AB}}\right)$ can be safely reduced to one or two.

Case 2: Now let $\ell_{\mathrm{B}}$ be odd, that is reduced to one, and let one of $\ell_{\mathrm{A}}$ or $\ell_{\varnothing}$ be also odd, that is reduced to one, and the other one even, that is reduced to two. In order to cover the unpaired leaf in $\Upsilon\left(L_{\mathrm{A}}\right)$ or $\Upsilon\left(L_{\varnothing}\right)$, the number of leaves in $\Upsilon\left(L_{\mathrm{AB}}\right)$ can then be safely reduced to two or three.

Case 3: Finally, we have that $\ell_{\mathrm{A}}$ is even and can be safely reduced to two. Furthermore, at least one value among $\ell_{\mathrm{B}}$ and $\ell_{\varnothing}$ is also even and can be safely reduced to two.

- Suppose first that either $\ell_{\mathrm{B}}$ or $\ell_{\varnothing}$ is odd, being reduced to one. Then the number of leaves in $\Upsilon\left(L_{\mathrm{AB}}\right)$ can be safely reduced to three or four.

- Now suppose that both $\ell_{\mathrm{B}}$ and $\ell_{\varnothing}$ are even, being reduced to two. Then the number of leaves in $\Upsilon\left(L_{\mathrm{AB}}\right)$ can be safely reduced to four or five. But actually we can still reduce it from five to three: suppose that the unpaired (fifth) leaf of subtree $\Upsilon\left(L_{\mathrm{AB}}\right)$ is covered by an out-traversal to one of the other three subtrees. At most at the same cost we could replace it by an in-traversal of $\Upsilon\left(L_{\mathrm{AB}}\right)$. Therefore in this case $\Upsilon\left(L_{\mathrm{AB}}\right)$ can be safely reduced to three or four.

The following proposition helps us to complete the proof of correctness of our approach.

Proposition 5: Any tagged component tree $T_{\circ}(\alpha, \beta)$ has an optimal cover that includes no bridge (a path connecting two internal nodes of $\left.T_{\circ}(\alpha, \beta)\right)$.

Proof: Any indel-neutral bridge can be elongated to connect two leaves with at most the same cost. We can then assume that, if an optimal cover must include a bridge, it is indel-saving. The cases of clean leaves only or leaves that share one tag are covered by Theorems 3 and 4.

Now we will prove the case of a component tree with two non-empty canonical subtrees whose leaves share no tag. By generalizing the statements of Theorems 3 and 4 , we can assume that there is an optimal cover that has no bridge connecting two nodes that belong to the same canonical subtree. Therefore, such a bridge either connects one node of each subtree to one node of the other, or if there is bad link $P$ between the subtrees, it can connect two nodes from $P$ or a node from $P$ and a node from one of the two subtrees. But, if we have such a bridge, we 
have no out-traversal nor semi-traversal, otherwise these would cover the same nodes covered by the bridge. This means that all the leaves of the two canonical subtrees are then covered by in-traversals or paths connecting a leaf to an internal node of the same canonical subtree. We then have two types of cases:

(i) $\Upsilon\left(L_{\mathrm{A}}\right)$ and $\Upsilon\left(L_{\mathrm{B}}\right)$ - such an indel-saving bridge would lead to an overall cost of $w=\left\lceil\ell_{\mathrm{A}} / 2\right\rceil+\left\lceil\ell_{\mathrm{B}} / 2\right\rceil+1$. If instead we replace this bridge by an indel-saving out-semitraversal, we get the cost of $w^{\prime}=\left\lceil\left(\ell_{\mathrm{A}}-1\right) / 2\right\rceil+\left\lceil\ell_{\mathrm{B}} / 2\right\rceil+1 \leqslant w$.

(ii) $\Upsilon\left(L_{\mathrm{A}}\right)$ and $\Upsilon\left(L_{\varnothing}\right)$ : (a) $\ell_{\varnothing}$ is odd - such an indel-saving bridge would lead to an overall cost of $w=\left\lceil\ell_{\mathrm{A}} / 2\right\rceil+$ $\ell_{\varnothing}(+1)+1$ and can be replaced by an indel-neutral out-traversal, resulting in the cost of $w^{\prime}=\left\lceil\left(\ell_{\mathrm{A}}-1\right) / 2\right\rceil+\ell_{\varnothing}-$ $1+2=\left\lceil\left(\ell_{\mathrm{A}}-1\right) / 2\right\rceil+\ell_{\varnothing}+1 \leqslant w ;(\mathrm{b}) \ell_{\varnothing}$ is even and $L_{\mathrm{A}} \geqslant 2$ - such an indel-saving bridge would lead to an overall cost of $w=\left\lceil\ell_{\mathrm{A}} / 2\right\rceil+\ell_{\varnothing}+1$ and can be replaced by two indel-neutral out-traversals, resulting in the cost of $w^{\prime}=\left\lceil\left(\ell_{\mathrm{A}}-2\right) / 2\right\rceil+\ell_{\varnothing}-2+4=\left\lceil\ell_{\mathrm{A}} / 2\right\rceil+\ell_{\varnothing}+1=w ;$ (c) $\ell_{\varnothing}$ is even and $L_{\mathrm{A}}=1-$ such an indel-saving bridge would lead to an overall cost of $w=1+\ell_{\varnothing}+1=\ell_{\varnothing}+2$ and can be replaced by one indel-neutral out-traversal, resulting in the cost of $w^{\prime}=\ell_{\varnothing}+2=w$.

For trees with three or four types of leaves we can develop a similar proof, but with more cases to be analyzed.

Lemma 4 The time and space complexity of our procedure of computing the inversion-indel distance is quadratic in the number of markers $|G|+|A|+|B|$.

Proof: Let $n=|G|+|A|+|B|$. The relational graph $R(\alpha, \beta)$, its number of cycles, their indel-potentials and the components can be computed in $O(n)$ time [5], [7], [13]. The tagged component tree can also be constructed in $O(n)$ time [13]. For our transformation of the chained tagged component tree $T_{\square}(\alpha, \beta)$ into the tagged component tree $T_{\circ}(\alpha, \beta)$, we traverse $T_{\square}$ in a bottom-up manner and recursively perform flower-contractions. For each removed tagged good leaf, its tag set is added to the one of its neighbor. While doing this, we obtain the sets of leaves $L_{\mathrm{A}}$, $L_{\mathrm{B}}, L_{\varnothing}$ and $L_{\mathrm{AB}}$.

We then need to obtain the non-empty canonical subtrees and bad links separating them. For each leaf class $L$, we again traverse the tree in a bottom-up manner. For each leaf in $L$ we proceed until we find a branching vertex, that we will call subroot of $L$. After all leaves of $L$ are visited, we repeat the procedure for all subroots of disjoint subtrees of $L$, until we get a single connected subtree. After repeating this procedure for all non-empty leaf classes, the bad vertices that were not visited are then bad links. All traversals of the tree can be done in $O(k)$ time, where $k \leqslant n$ is the number of nodes of $T_{\circ}(\alpha, \beta)$.

For the reduction, we can obtain the set of balanced in-traversals of each canonical subtree in $O(k)$ time [14]. The reduction of each tagged canonical subtree requires another traversal of the tree and also takes $O(k)$ time.

After reducing the tagged canonical subtrees, but before performing the reduction of the clean subtree $\Upsilon\left(L_{\varnothing}\right)$ we still need to examine whether the tree has the solo leaf. The candidates can be found by detecting leaves in short leaf-branches, a procedure already described in [13], but we still need to check for each candidate whether it is indeed a solo leaf. The only approach we could devise so far tests all possible hypotheses (no solo leaf and each clean short leaf-branch of $T_{\circ}(\alpha, \beta)$ as solo leaf) and takes one that results in the smallest cover cost. In order to get the cover cost of each possible residual subtree, we need to examine its topology. Non-canonical partition subtrees and tag mates can be obtained by a single traversal of the residual tree, that takes $O(k)$ time. Once we have obtained all required properties, we can look up the optimal cover cost at the residual leaf composition enumeration (in Appendix 2). Therefore, the step of determining the solo leaf together with reducing the clean subtree and obtaining the cover of the residual tree has time complexity $O(k \ell)$, where $\ell$ is the number of leaves in $\Upsilon\left(L_{\varnothing}\right)$. Since $\ell \leqslant k \leqslant n$, the time complexity of this step is $O\left(n^{2}\right)$ and it dominates the complexity of the whole procedure.

In summary, the time complexity of our approach is $O\left(n^{2}\right)$. 


\section{Algorithms}

Algorithm 2 computes the residual tree of any tagged component tree based on Reduction Bounds 1 to 5 We observe that the reduction of tagged subtrees do not affect the solo leaf candidates. In fact a reduction can increase the length of a leaf-branch, turning a short into a long leaf-branch, as shown in Figure 10. However, this is a very limited behaviour that only happens when the reduction is not balanced, as in the reduction from 3 to one leaf, and can be prevented by the choice of the leaf to be kept in this reduction (see more details below). Therefore it is safe to detect the solo leaf after reducing all tagged subtrees. 


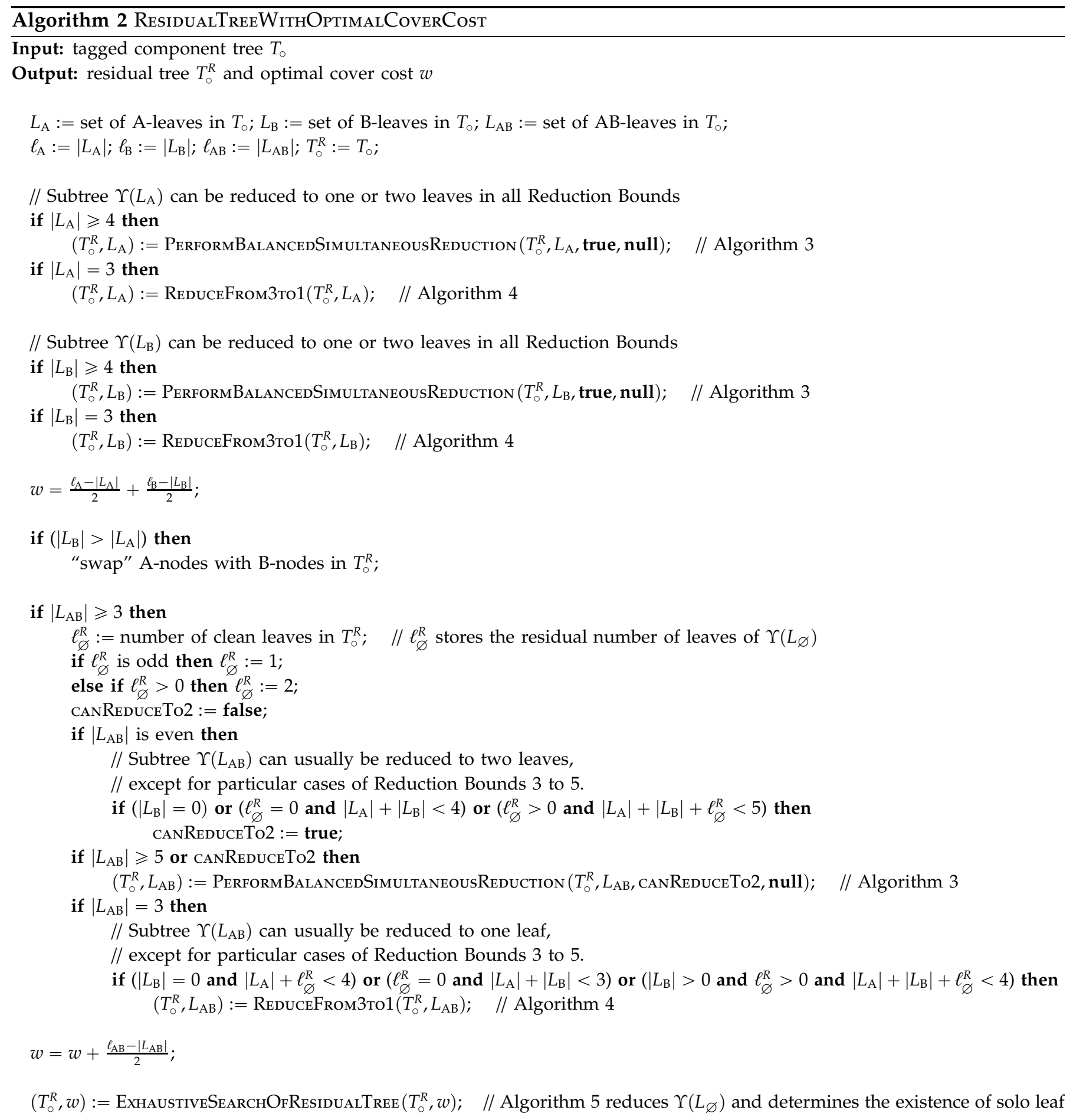


Reducing a canonical subtree from $\ell \geqslant 4$ to 2 (if $\ell$ is even) or 3 (if $\ell$ is odd) is described in Algorithm 3 it suffices to take any set of balanced in-traversals (keeping the solo leaf if the reduced subtree is clean). In fact, this algorithm uses a generalization of a balanced reduction. Let $\mathcal{P}$ be a set of balanced in-traversals of some canonical subtree of $T_{\circ}(\alpha, \beta)$. A simultaneous $\mathcal{P}$-reduction of $T_{\circ}(\alpha, \beta)$ results in a smaller tagged component tree as follows. Let $T^{\prime}$ be the tagged tree obtained from $T_{\circ}(\alpha, \beta)$ by replacing each bad node $b$ of each $P \in \mathcal{P}$ by a good node $g$, such that $\operatorname{TAG}(g)=\operatorname{TAG}(b)$. Then $T_{\circ}^{-\mathcal{P}}(\alpha, \beta)$ is the flower-contracted version of $T^{\prime}$.

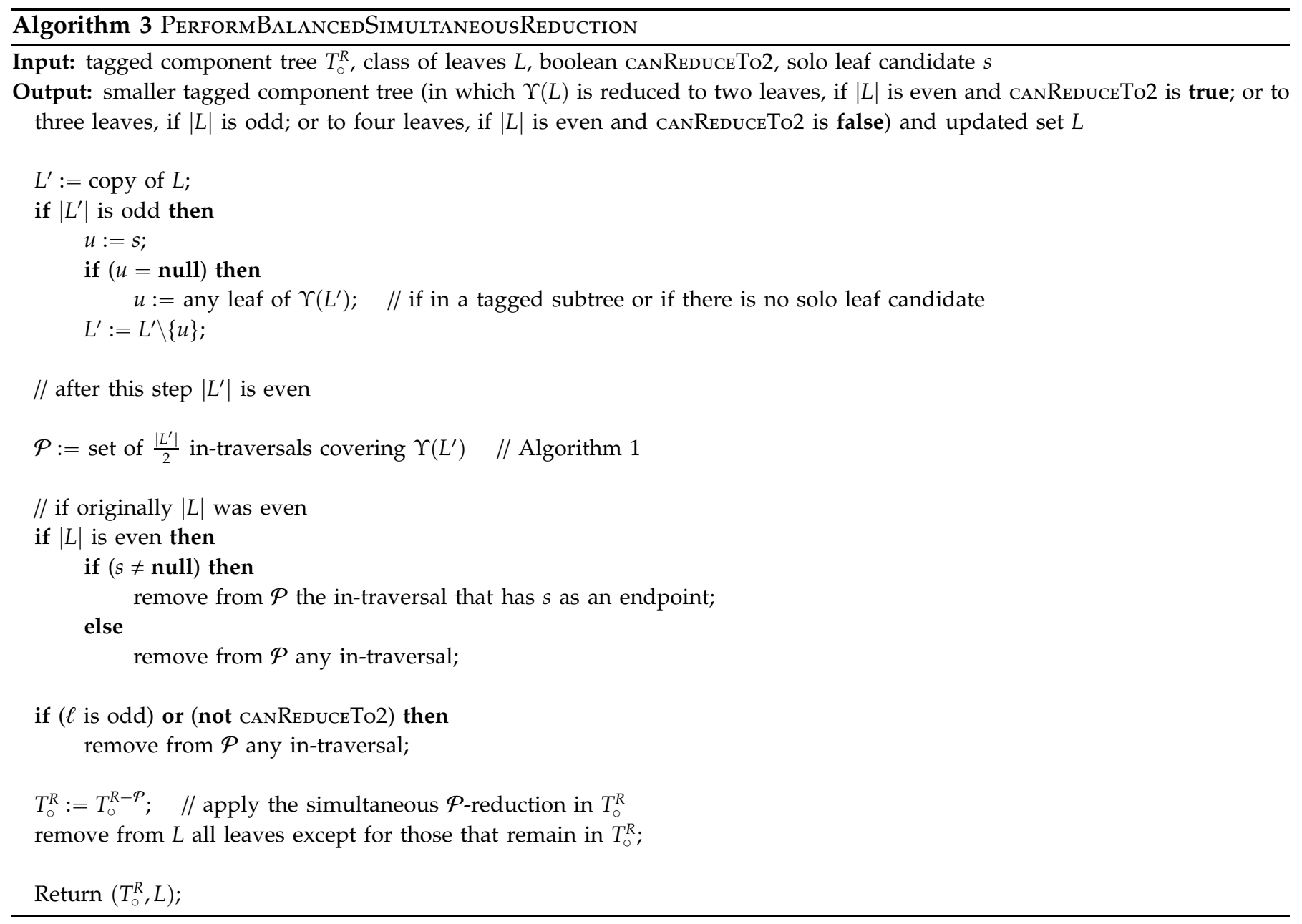

Reducing a canonical subtree from $\ell=3$ to 1 is described in Algorithm 4 here we need to take one leaf to be kept, while the other two are used for the reduction. In order to prevent the risk of creating a new bad leaf with the reduction and/or the risk of selecting an unsafe reduction, it is necessary to be careful with the decision of the leaf to be kept, that we call essential leaf. Sometimes two or even the three leaves are suitable to be essential, but, since it suffices to identify a single one, the first one that is found is called the essential leaf. It is identified as follows:

- It is the leaf of a leaf-branch of $\Upsilon(L)$ that is also a leaf-branch of $T_{\circ}(\alpha, \beta)$, if there is any. This case avoids the "destruction" of a possible solo leaf, as illustrated in Figure 10 It also avoids other unsafe reductions, as illustrated in Figure 11, parts (i) and (ii).

- Otherwise at least one node of each leaf-branch $P_{i}$ of $\Upsilon(L)$ is connected to $T_{\circ}(\alpha, \beta) \backslash \Upsilon(L)$; then let $F\left(P_{i}\right)$ be the subgraph of $T_{\circ}(\alpha, \beta) \backslash \Upsilon(L)$ that is connected to the leaf-branch $P_{i}$ and the set $\mathcal{S}\left(P_{i}\right)$ be the superset of the tag sets of the leafs of $T_{\circ}(\alpha, \beta)$ that are in $F\left(P_{i}\right)$. If, for two leaf-branches $P_{1}$ and $P_{2}$ of $\Upsilon(L)$, we have $\mathcal{S}\left(P_{1}\right) \cap \mathcal{S}\left(P_{2}\right) \neq \varnothing$, then any of the two leaves in $P_{1}$ or $P_{2}$ is the essential leaf. This case avoids that a canonical subtree become isolated, as illustrated in Figure 11, part (iii).

- Otherwise any leaf of $\Upsilon(L)$ is the essential leaf. 

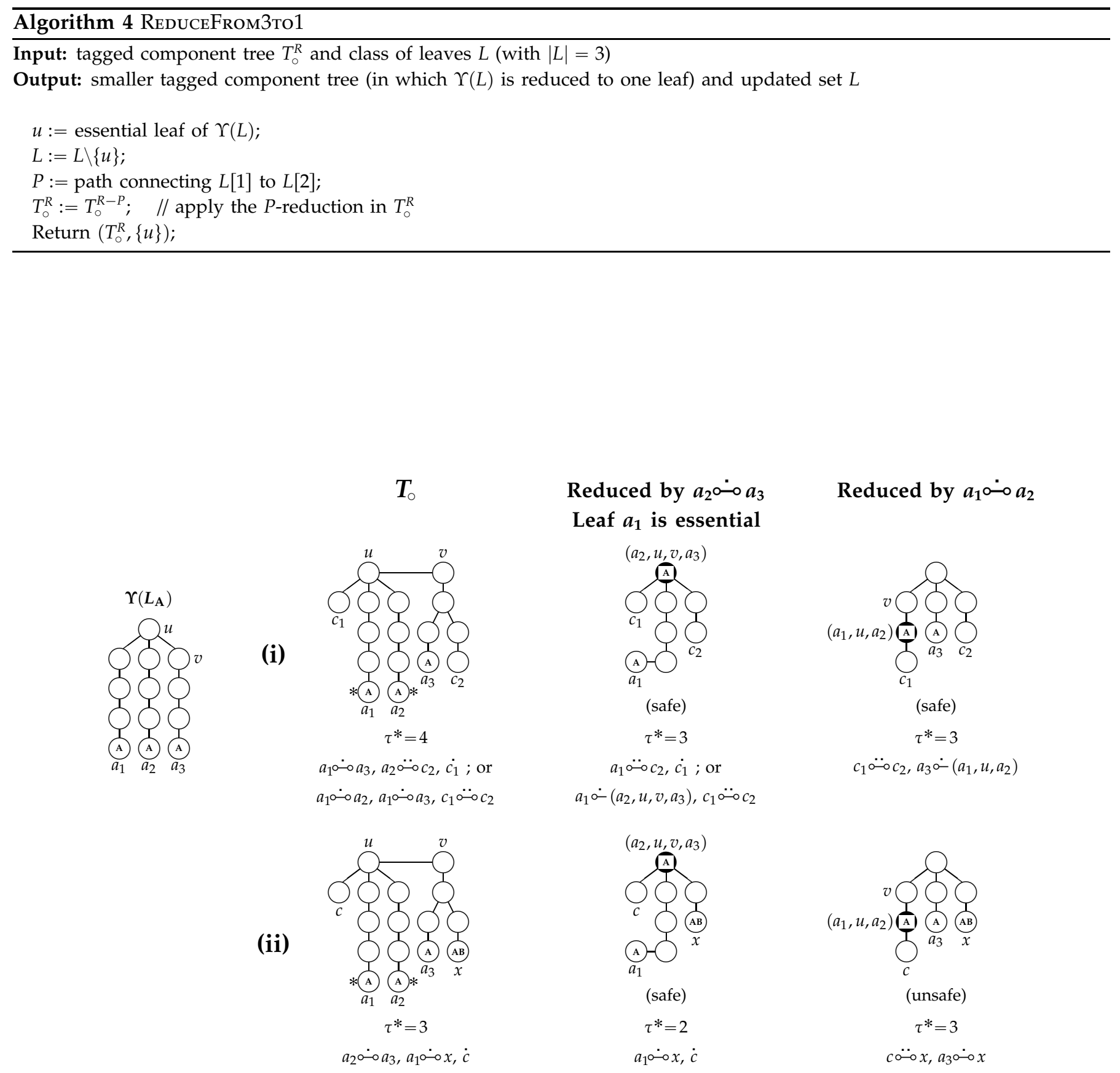

Fig. 10. Examples showing that a careless reduction on $\Upsilon\left(L_{\mathrm{A}}\right)$ can "destroy" a solo leaf. In (i) the given $T_{\circ}$ has an optimal cover with a short path covering clean leaf $c_{1}$. This solo leaf in non-mandatory: there is also an alternative optimal cover including only traversals. After the reduction $a_{1} \circ{ }_{-} \circ a_{2}$, the leaf $c_{1}$ is in a long leaf-branch and cannot be covered by a short path, nevertheless this reduction is safe. In (ii) we have a $T_{\circ}$ with a mandatory solo leaf. Again, after the reduction $a_{1} \circ \circ a_{2}$, the leaf $c_{1}$ is in a long leaf-branch and cannot be covered by a short path, and in this case the reduction is indeed unsafe. Note that this can only happen when the reduction takes an in-traversal connecting consecutive leaves in the assumed circular order, and this is only possible when reducing from 3 to 1 . Fortunately, this side effect is easily prevented by the choice of the essential leaf. 


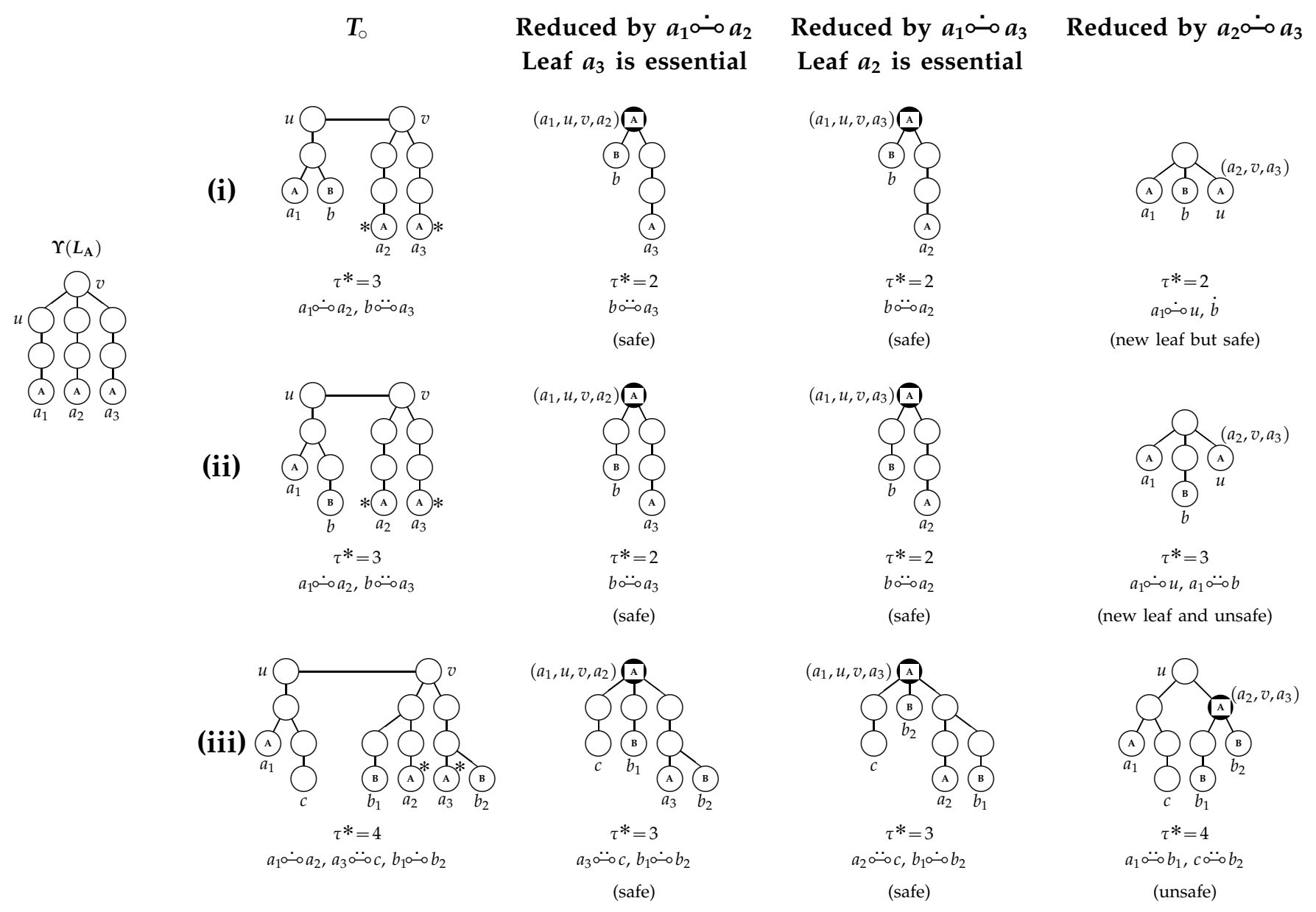

Fig. 11. Examples of reduction of canonical subtree $\Upsilon\left(L_{\mathrm{A}}\right)$ from 3 leaves to one leaf. In all examples the possible essential leaves are $a_{2}$ and $a_{3}$, marked with $\mathrm{a} *$. In (i) and (ii) only the leaf-branch ending in $a_{1}$ intersects $T_{\circ}$. Indeed, if $a_{1}$ is kept by the reduction, node $u$ becomes a leaf in the reduced tree, and the reduction can be safe or unsafe. If any of the possible essential leaves is kept, the number of leaves is reduced by two and the reduction is safe. In (iii) all three leaf-branches of $\Upsilon\left(L_{\mathrm{A}}\right)$ intersect $T_{0}$. Since two branches intersect a leaf-branch of $\Upsilon\left(L_{\mathrm{B}}\right)$, their respective leaves are the possible essential leaves. The reduction is indeed safe if one of these two leaves is kept, otherwise it is unsafe. 
The only approach we could devise for determining the existence of the solo leaf tests all possible hypotheses (no solo leaf and each clean short leaf-branch of $T_{\circ}(\alpha, \beta)$ as solo leaf) and takes one that results in the smallest cover cost. For that reason, reducing the clean subtree is done together with searching the solo leaf, as described in Algorithm 5

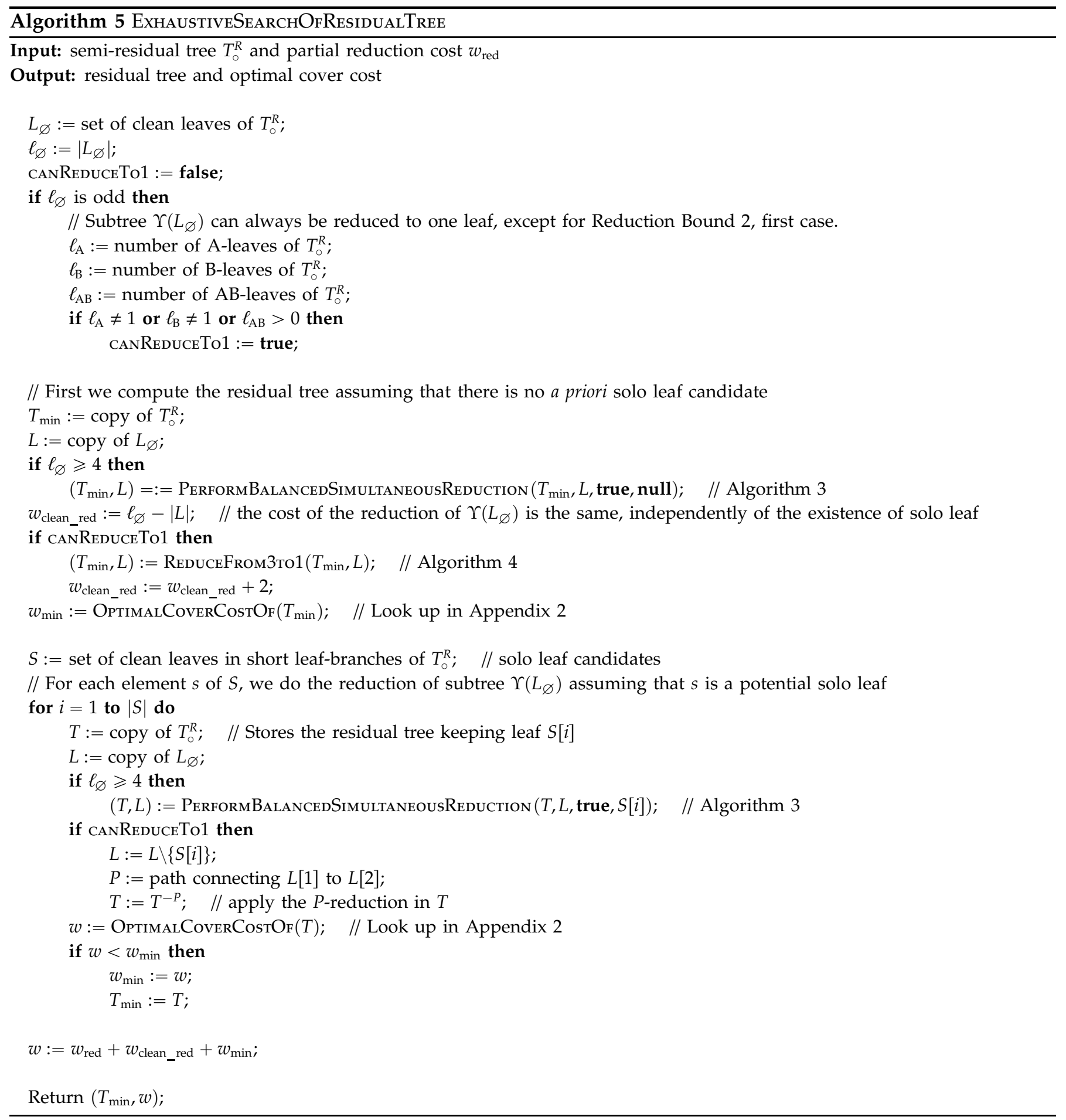




\section{Appendix 2: Enumeration of Optimal Covers for Residual Trees}

In this section we enumerate optimal covers and their respective costs for all topologies of residual trees that have at least one leaf with non-empty tag set and at least two leaves that do not share a tag. Let $\ell_{\mathrm{A}}$ be the number of A-leaves, $\ell_{\mathrm{B}}$ be the number of B-leaves, $\ell_{\varnothing}$ be the number of $\varnothing$-leaves and $\ell_{\mathrm{AB}}$ be number of AB-leaves in a residual tree. Recall that $\ell_{\mathrm{A}} \leqslant 2$ for all residual trees. Due to the symmetry between A-nodes and B-nodes, we assume without loss of generality that $\ell_{\mathrm{A}} \geqslant \ell_{\mathrm{B}}$. The enumeration is kept as extensive as necessary but as simple as possible.

\section{Residual trees are identified by their leaf composition}

A leaf composition is represented by a tuple $\left(\ell_{\mathrm{A}}, \ell_{\mathrm{B}}, \ell_{\varnothing}, \ell_{\mathrm{AB}}\right)$ of four non negative values that always has $\ell_{\mathrm{A}}$ in the first position, $\ell_{\mathrm{B}}$ in the second position, $\ell_{\varnothing}$ in the third position and $\ell_{\mathrm{AB}}$ in the fourth position.

Examples:

$(0,0,2,1)$ : A leaf composition in which subtrees $\Upsilon\left(L_{A}\right)$ and $\Upsilon\left(L_{B}\right)$ are empty, subtree $\Upsilon\left(L_{\varnothing}\right)$ has two leaves and subtree $\Upsilon\left(L_{\mathrm{AB}}\right)$ has one leaf.

$(2,1,2,4)$ : A leaf composition in which subtree $\Upsilon\left(L_{\mathrm{A}}\right)$ has two leaves, subtree $\Upsilon\left(L_{\mathrm{B}}\right)$ has one leaf, subtree $\Upsilon\left(L_{\varnothing}\right)$ has two leaves and subtree $\Upsilon\left(L_{\mathrm{A}}\right)$ has four leaves.

\section{Nomenclature}

General topologies of residual trees

$\Upsilon_{\bowtie}$ : The tagged component tree $T_{\circ}^{R}$ is fully co-rooted.

$\Upsilon_{\Leftrightarrow}:$ The tagged component tree $T_{\circ}^{R}$ is fully separated.

$\gtrless_{0}$ : There is a short leaf-branch in $T_{0}^{R}$ with a clean leaf.

Relations between partition subtrees are described by the following notation, presented with the help of examples.

Partition subtrees and their specific relations in possible topologies of residual trees

$\Upsilon_{\mathrm{A}}$ : $\quad$ The canonical subtree $\Upsilon\left(L_{\mathrm{A}}\right)$.

$\Upsilon_{\varnothing} \Upsilon_{\mathrm{B}}$ : The partition subtree $\Upsilon\left(L_{\varnothing} \cup L_{\mathrm{B}}\right)$, in which the canonical subtrees $\Upsilon\left(L_{\varnothing}\right)$ and $\Upsilon\left(L_{\mathrm{B}}\right)$ may be co-rooted or separated.

$\Upsilon_{\mathrm{A}} \bowtie \Upsilon_{\mathrm{AB}}$ : The partition subtree $\Upsilon\left(L_{\mathrm{A}} \cup L_{\mathrm{AB}}\right)$, in which the subtrees $\Upsilon\left(L_{\mathrm{A}}\right)$ and $\Upsilon\left(L_{\mathrm{AB}}\right)$ are co-rooted.

$\Upsilon_{\varnothing} \bowtie \bar{\Upsilon}: \quad$ The subtree $\Upsilon\left(L_{\varnothing}\right)$ is non-isolated, that is, $\Upsilon\left(L_{\varnothing}\right)$ and its complementary subtree $\bar{\Upsilon}\left(L_{\varnothing}\right)=\Upsilon\left(L_{\mathrm{A}} \cup\right.$ $\left.L_{\mathrm{B}} \cup L_{\mathrm{AB}}\right)$ are co-rooted.

$\Upsilon_{B} \Leftrightarrow \Upsilon_{\varnothing}: \quad$ The partition subtree $\Upsilon\left(L_{B} \cup L_{\varnothing}\right)$, in which the subtree $\Upsilon\left(L_{B}\right)$ is separated from $\Upsilon\left(L_{\varnothing}\right)$.

$\Upsilon_{\mathrm{A}} \Leftrightarrow \bar{\Upsilon}$ : The subtree $\Upsilon\left(L_{\mathrm{A}}\right)$ is isolated, that is, $\Upsilon\left(L_{\mathrm{A}}\right)$ is separated from its complementary subtree $\bar{\Upsilon}\left(L_{\mathrm{A}}\right)=$ $\Upsilon\left(L_{\mathrm{B}} \cup L_{\mathrm{AB}} \cup L_{\varnothing}\right)$.

$\mathrm{A} \vdash \Upsilon_{\varnothing}: \quad$ The extended subtree $\Upsilon^{+}\left(L_{\varnothing}\right)$ has an A-mate.

₹*: $\quad$ The tree obtained by pruning from $T_{\circ}^{R}$ a clean short leaf-branch.

$\Upsilon_{\mathrm{A}} \bowtie \bar{f}_{\circ}: \quad$ The subtree $\Upsilon\left(L_{\mathrm{A}}\right)$ is non-isolated in a $\Upsilon_{\circ}$.

The relations above can be applied to non-canonical subtrees:

$$
\left(\Upsilon_{A} \smile \Upsilon_{B}\right) \bowtie \bar{\Upsilon}, \quad \Upsilon_{\varnothing} \bowtie\left(\Upsilon_{A} \Leftrightarrow \Upsilon_{B}\right), \quad\left(\Upsilon_{A B} \bowtie \Upsilon_{B}\right) \Leftrightarrow \Upsilon_{A}, \quad\left(\Upsilon_{A B} \sim \Upsilon_{\varnothing}\right) \Leftrightarrow \bar{\Upsilon}, \quad A \vdash\left(\Upsilon_{B} \smile \Upsilon_{\varnothing}\right), \quad A \vdash\left(\Upsilon_{B} \bowtie \Upsilon_{\varnothing}\right)
$$


Identifiers of the optimal covers

I, II: Basic optimal covers

M: $\quad$ Tag mate

S: $\quad$ Short path (usually covering a solo leaf)

nR: Not reducible

»: Further reduction is possible for this type of topology

$\mathrm{W}$ : Worst case

Optimal covers are described by the following notation, presented here with the help of examples.

\section{Descriptions of optimal covers}

(A): $\quad$ A short path (whose cost is one) covering an A-leaf.

$\Upsilon_{\mathrm{AB}} \circ-\Upsilon_{\mathrm{AB}}$ : A covering in-traversal using two AB-leaves that have not been covered before. The dot on top indicates that the cost of this traversal is one.

$\Upsilon_{\varnothing} \because \ddot{\circ} \Upsilon_{A B}:$ A covering out-traversal using one $\varnothing$ - and one AB-leaf that have not been covered before. The dots on top indicate that the cost of this traversal is two.

$\Upsilon_{\mathrm{A}} \circ-\mathrm{-}\left(\Upsilon_{\mathrm{AB}}\right)$ : The parentheses around the subtree indicate that the respective leaf has already been covered previously.

$\Upsilon_{\mathrm{AB}} \mathrm{O}^{\circ} \mathrm{A}$ : $\quad$ A semi-traversal (of cost one) using an AB-leaf with an internal node that is an A-mate. The specific (extended) subtree that contains the A-mate is given in the topology description.

| 2: $\quad$ The cost of the cover is given to the right of the vertical bar.

$\cup(2,2,2,1)$ : The optimal cover in this case consists of the given in-traversal plus the cost of the optimal cover of the appropriate topology of the reduced leaf composition $(2,2,2,1)$.

$\mid+1$ : $\quad$ A " + " means that the cost has to be added to that of the reduced leaf composition.

For each leaf composition and topology we describe one optimal cover, even though other optimal covers may also exist.

\section{Enumeration of residual leaf compositions of Group 1}

Residual leaf compositions with exactly two non-empty canonical subtrees that do not share a tag, defined by Reduction Bound 1. This includes 11 leaf compositions, divided in three subgroups:

\begin{tabular}{cc|cc|cc}
\hline \multicolumn{6}{c}{ Reduction Bound 1 } \\
\hline \multicolumn{2}{c}{$\left(\ell_{\mathrm{A}}, \ell_{\mathrm{B}}, 0,0\right)$} & \multicolumn{2}{c}{$\left(\ell_{\mathrm{A}}, 0, \ell_{\varnothing}, 0\right)$} & \multicolumn{2}{c}{$\left(0,0, \ell_{\varnothing}, \ell_{\mathrm{AB}}\right)$} \\
\hline$(1,1,0,0)$ & $(2,1,0,0)$ & $(1,0,1,0)$ & $(2,0,1,0)$ & $(0,0,1,1)$ & $(0,0,2,1)$ \\
& $(2,2,0,0)$ & $(1,0,2,0)$ & $(2,0,2,0)$ & $(0,0,1,2)$ & $(0,0,2,2)$ \\
\hline
\end{tabular}

Residual leaf compositions of subgroup $\left(\ell_{\mathrm{A}}, \ell_{\mathrm{B}}, 0,0\right)$

$(1,1,0,0)$

$$
\text { any topology I } \Upsilon_{\mathrm{A}} \stackrel{\sim}{\circ} \circ \Upsilon_{\mathrm{B}} \mid 2
$$

$(2,1,0,0)$

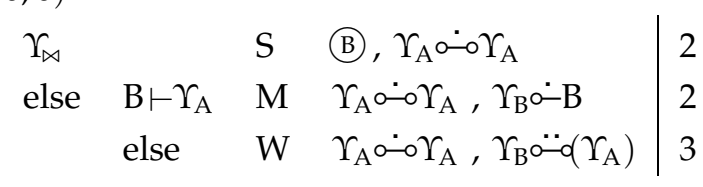


$(2,2,0,0)$

\begin{tabular}{|c|c|c|c|}
\hline$\Upsilon_{\bowtie}$ & & $\mathrm{I}$ & $\Upsilon_{A} \circ \dot{\circ} \circ \Upsilon_{A}, \Upsilon_{B} \circ \circ \Upsilon_{B}$ \\
\hline short bad link & & $\mathrm{S}$ & $\Upsilon_{A} \circ-\Upsilon_{A}, \Upsilon_{B} \circ \circ \Upsilon_{B}$, bad link cut \\
\hline else & $\mathrm{A} \vdash \Upsilon_{\mathrm{B}}$ & $\mathrm{Ma}$ & $\Upsilon_{A} \circ \div \circ \Upsilon_{A},\left(\Upsilon_{A}\right) \circ-A, \Upsilon_{B} \circ \sim \Upsilon_{B}$ \\
\hline & $\mathrm{B} \vdash \Upsilon_{\mathrm{A}}$ & $\mathrm{Mb}$ & $\Upsilon_{A} \circ \dot{\circ} \circ \Upsilon_{A},\left(\Upsilon_{B}\right) \circ \dot{-B}, \Upsilon_{B} \circ \dot{\circ} \circ \Upsilon_{B}$ \\
\hline & else & W & $2 \times \Upsilon_{A} \cdots{ }^{\circ} \circ \Upsilon_{B}$ \\
\hline
\end{tabular}

Residual leaf compositions of subgroup $\left(\ell_{A}, 0, \ell_{\varnothing}, 0\right)$

$(1,0,1,0)$ any topology I $\Upsilon_{A} \circ \ddot{\circ}_{\varnothing} \Upsilon_{\varnothing} \mid 2$

$(1,0,2,0)$

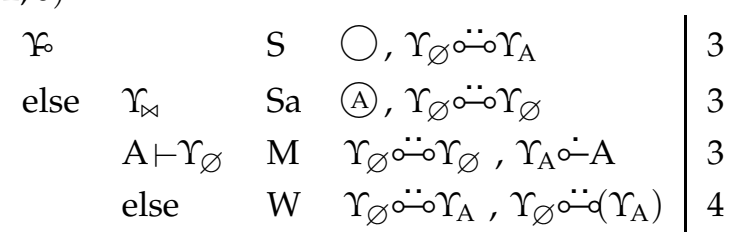

$(2,0,1,0)$

$$
\begin{array}{lll|l}
\Upsilon_{\triangleright} & S & \bigcirc, \Upsilon_{A} \circ \circ \Upsilon_{A} & 2 \\
\text { else } & W & \Upsilon_{\varnothing} \circ \circ \Upsilon_{A}, \Upsilon_{A} \circ-\circ\left(\Upsilon_{A}\right) & 3
\end{array}
$$

$(2,0,2,0)$

$$
\begin{array}{lll|l}
\Upsilon_{\bowtie} & \text { I } & \Upsilon_{\varnothing} \ddot{\circ} \circ \Upsilon_{\varnothing}, \Upsilon_{A} \circ \dot{\circ} \circ \Upsilon_{A} & 3 \\
\text { else } & W & 2 \times \Upsilon_{\varnothing} \cdots \Upsilon_{A} & 4
\end{array}
$$

\section{Residual leaf compositions of subgroup $\left(0,0, \ell_{\varnothing}, \ell_{\mathrm{AB}}\right)$}

$(0,0,1,1)$

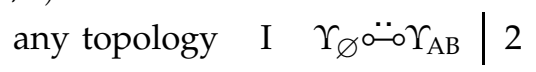

$(0,0,1,2)$

$$
\begin{array}{lll|l}
\Upsilon_{\bowtie} & S & \bigcirc, \Upsilon_{A B} \circ \dot{\circ} \circ \Upsilon_{A B} & 2 \\
\text { else } & W & \Upsilon_{\varnothing} \circ \ddot{\circ} \Upsilon_{A B}, \Upsilon_{A B} \circ-\circ\left(\Upsilon_{A B}\right) & 3
\end{array}
$$

$(0,0,2,1)$

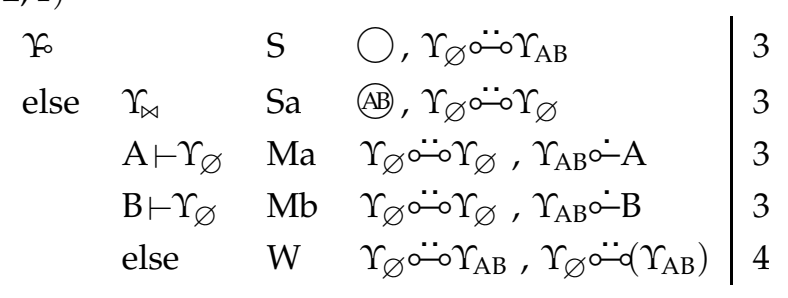

$(0,0,2,2)$

$$
\begin{array}{lll|l}
\Upsilon_{\bowtie} & I & \Upsilon_{\varnothing} \circ \circ \Upsilon_{\varnothing}, \Upsilon_{A B} \circ \circ \Upsilon_{A B} & 3 \\
\text { else } & W & 2 \times \Upsilon_{\varnothing} \circ \cdots \Upsilon_{A B} & 4
\end{array}
$$




\section{Enumeration of residual leaf compositions of Group 2}

Residual leaf compositions with exactly three non-empty canonical subtrees. This includes 25 leaf compositions, divided in three subgroups:

\begin{tabular}{c|cc|}
\hline \multicolumn{3}{c}{ Reduction Bound 2} \\
\hline Case 1 & \multicolumn{2}{c}{ Case 2} \\
$\left(1,1, \ell_{\varnothing}, 0\right)$ & $\left(2,1, \ell_{\varnothing}, 0\right)$ & $\left(2,2, \ell_{\varnothing}, 0\right)$ \\
\hline$(1,1,1,0)$ & $(2,1,1,0)$ & $(2,2,1,0)$ \\
$(1,1,2,0)$ & $(2,1,2,0)$ & $(2,2,2,0)$ \\
$(1,1,3,0)$ & & \\
& & \\
\hline
\end{tabular}

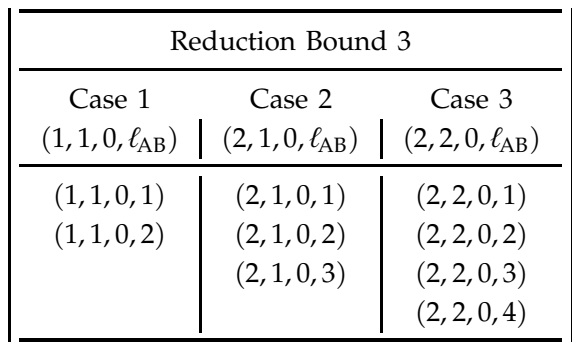

\begin{tabular}{|c|c|c|c|}
\hline \multicolumn{4}{|c|}{ Reduction Bound 4} \\
\hline$\left(1,0,1, \ell_{\mathrm{AB}}\right)$ & $\begin{array}{c}\text { Case } 1 \\
\left(1,0,2, \ell_{\mathrm{AB}}\right)\end{array}$ & $\left(2,0,1, \ell_{\mathrm{AB}}\right)$ & $\begin{array}{c}\text { Case } 2 \\
\left(2,0,2, \ell_{\mathrm{AB}}\right)\end{array}$ \\
\hline$(1,0,1,1)$ & $(1,0,2,1)$ & $(2,0,1,1)$ & $(2,0,2,1)$ \\
\hline \multirow[t]{2}{*}{$(1,0,1,2)$} & $(1,0,2,2)$ & $(2,0,1,2)$ & $(2,0,2,2)$ \\
\hline & & & $(2,0,2,3)$ \\
\hline
\end{tabular}

Reduction Bound 2, residual leaf compositions of subgroup $\left(1,1, \ell_{\varnothing}, 0\right)+\left(2,1, \ell_{\varnothing}, 0\right)+\left(2,2, \ell_{\varnothing}, 0\right)$

$(1,1,1,0)$

$$
\begin{aligned}
& \Upsilon_{\varnothing \bowtie \bar{\Upsilon}} \\
& \text { else } \Upsilon_{\mathrm{A}} \bowtie \bar{\Upsilon} \\
& \Upsilon_{B} \bowtie \bar{\Upsilon} \\
& \text { else } \\
& \begin{array}{lll|l}
\text { S } & \bigcirc, \Upsilon_{A} \cdots{ }^{\circ} \circ \Upsilon_{B} & 3 \\
\text { Sa } & \text { (A), } \Upsilon_{B} \circ \cdots \Upsilon_{\varnothing} & 3
\end{array} \\
& \mathrm{Sb} \text { (B), } \Upsilon_{A} \circ \ddot{\circ}^{\circ} \Upsilon_{\varnothing} \quad 3 \\
& \mathrm{~A} \vdash\left(\Upsilon_{B} \Leftrightarrow \Upsilon_{\varnothing}\right) \quad \text { Ma } \Upsilon_{B} \circ \ddot{\circ} \circ \Upsilon_{\varnothing}, \Upsilon_{A} \circ-A \quad \text { A }
\end{aligned}
$$

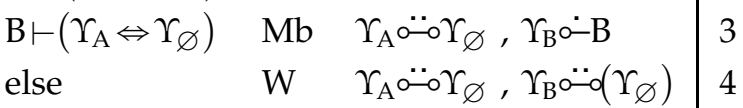

$(1,1,2,0)$

any topology I $\Upsilon_{A} \circ \ddot{\circ} \circ \Upsilon_{\varnothing}, \Upsilon_{\varnothing} \circ \ddot{\circ}_{\circ} \Upsilon_{B} \mid 4$

$(1,1,3,0)$

$$
\begin{aligned}
& \Upsilon_{0} \wedge \Upsilon_{A} \Leftrightarrow \bar{\Upsilon} \wedge \Upsilon_{B} \Leftrightarrow \bar{\Upsilon} \\
& \wedge \bar{A} F\left(\Upsilon_{B} \Leftrightarrow \Upsilon_{Q}\right) \wedge \bar{B} F\left(\Upsilon_{A} \Leftrightarrow \Upsilon_{Q}\right) \\
& \text { S } \bigcirc, \Upsilon_{A} \cdots \Upsilon_{\varnothing}, \Upsilon_{B} \circ \cdots \Upsilon_{\varnothing} \mid 5 \\
& \ggg \Upsilon_{\varnothing} \circ \ddot{\circ} \circ \Upsilon_{\varnothing} \cup(1,1,1,0) \mid+2
\end{aligned}
$$

$(2,1,1,0)$

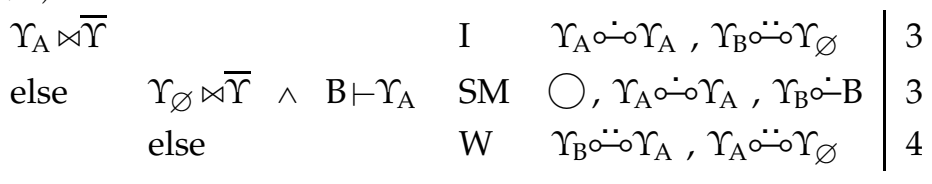

$(2,1,2,0)$

$$
\begin{aligned}
& \Upsilon_{\bowtie} \\
& \exists \Upsilon_{f_{0}} \quad \Upsilon_{A} \bowtie \bar{f}_{\circ} \\
& \Upsilon_{A} \bowtie \bar{\Upsilon} \quad \Upsilon_{\varnothing} \Leftrightarrow \bar{\Upsilon} \quad B \vdash \Upsilon_{\varnothing}
\end{aligned}
$$

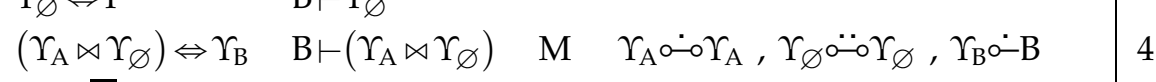

$$
\begin{aligned}
& \Upsilon_{\mathrm{A}} \Leftrightarrow \bar{\Upsilon} \quad \Upsilon_{\varnothing} \bowtie \bar{\Upsilon} \quad \mathrm{B} \vdash \Upsilon_{\mathrm{A}} \\
& \begin{array}{ll|l}
\mathrm{Sb} \quad \text { (B) }, \Upsilon_{\varnothing} \circ \ddot{\circ} \circ \Upsilon_{\varnothing}, \Upsilon_{\mathrm{A}} \circ \cdots \Upsilon_{\mathrm{A}} & 4
\end{array} \\
& \text { S } \bigcirc, \Upsilon_{\varnothing} \circ \circ \Upsilon_{B}, \Upsilon_{A} \circ \circ \Upsilon_{A} \quad 4 \\
& \text { W } \Upsilon_{A} \circ \circ \Upsilon_{A}, \Upsilon_{B} \circ \ddot{\circ} \Upsilon_{\varnothing}, \Upsilon_{\varnothing} \ddot{\circ}\left(\Upsilon_{A}\right) \mid 5
\end{aligned}
$$


$(2,2,1,0)$

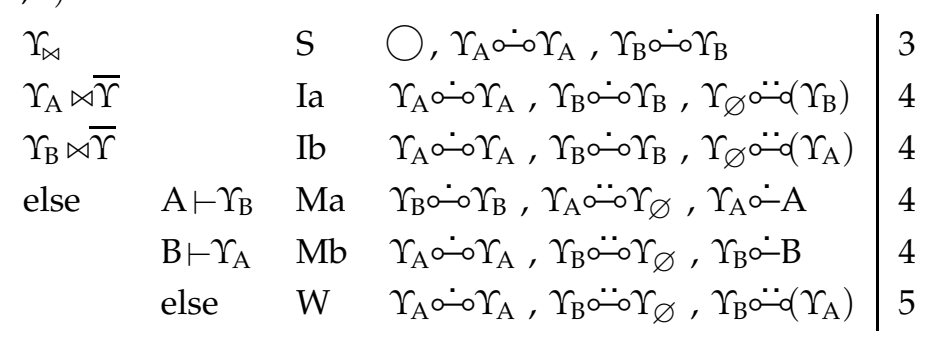

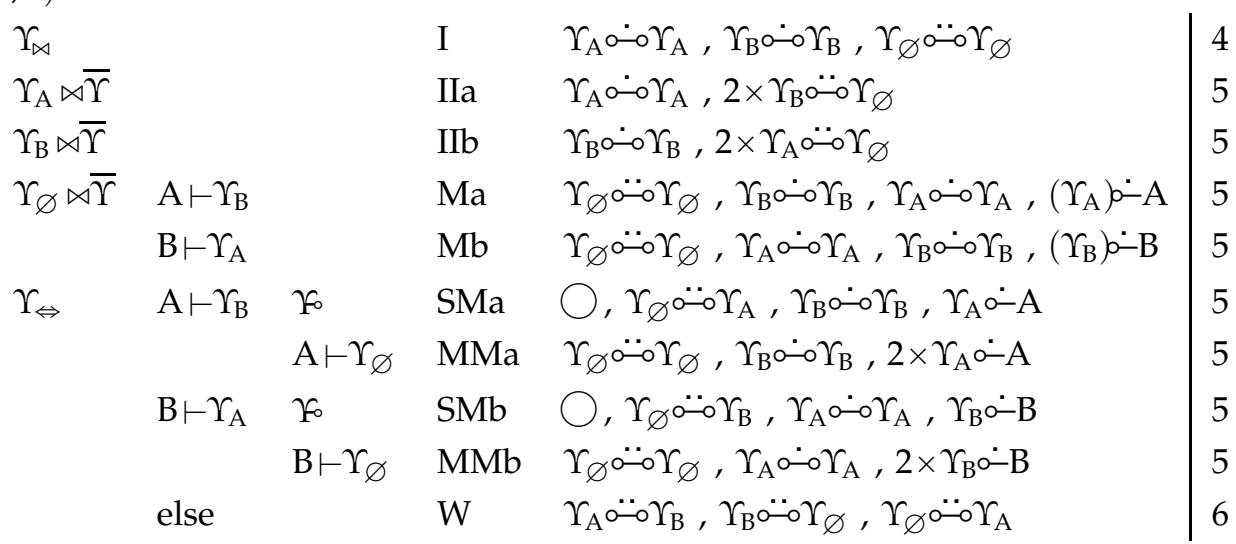

Reduction Bound 3, residual leaf compositions of subgroup $\left(1,1,0, \ell_{A B}\right)+\left(2,1,0, \ell_{A B}\right)+\left(2,2,0, \ell_{A B}\right)$

$(1,1,0,1)$ any topology I $\Upsilon_{A} \circ \dot{\circ} \circ \Upsilon_{A B}, \Upsilon_{B} \circ \cdot-\left(\Upsilon_{A B}\right) \mid 2$

$(1,1,0,2)$

$$
\text { any topology I } \Upsilon_{\mathrm{A}} \circ \dot{\circ} \circ \Upsilon_{\mathrm{AB}}, \Upsilon_{\mathrm{AB}} \circ \dot{\circ} \Upsilon_{\mathrm{B}} \mid 2
$$

$$
\begin{array}{lll|l}
\Upsilon_{A} \bowtie \bar{\Upsilon} & I & \Upsilon_{A} \circ \dot{\circ} \circ \Upsilon_{A}, \Upsilon_{A B} \circ \cdots \Upsilon_{B} & 2 \\
\text { else } & W & \Upsilon_{A B} \circ \sim \Upsilon_{A}, \Upsilon_{A} \circ \cdots \Upsilon_{B} & 3
\end{array}
$$

$(2,1,0,2)$

$$
\text { any topology I } 2 \times \Upsilon_{A} \circ-\circ \Upsilon_{A B}, \Upsilon_{B} \circ-0\left(\Upsilon_{A B}\right) \mid 3
$$

$(2,1,0,3)$

$$
\begin{array}{llll|l}
\Upsilon_{\mathrm{A}} \Leftrightarrow \bar{\Upsilon} & \mathrm{nR} & \Upsilon_{\mathrm{B}} \circ \circ \Upsilon_{\mathrm{AB}}, 2 \times \Upsilon_{\mathrm{A}} \circ \circ \Upsilon_{\mathrm{AB}} & 3 \\
\text { else } & \ggg & \Upsilon_{\mathrm{AB}} \circ \circ \Upsilon_{\mathrm{AB}} \cup(2,1,0,1) & +1
\end{array}
$$

$$
\begin{aligned}
& \Upsilon_{A} \bowtie \bar{\Upsilon} \quad \text { Ia } \Upsilon_{A} \circ \cdots \Upsilon_{A}, \Upsilon_{B} \circ \circ \Upsilon_{A B}, \Upsilon_{B} \circ-0\left(\Upsilon_{A B}\right) \mid 3 \\
& \Upsilon_{B} \bowtie \bar{\Upsilon} \quad \mathrm{Ib} \quad \Upsilon_{B} \circ \cdots \Upsilon_{B}, \Upsilon_{A} \circ \cdots \Upsilon_{A B}, \Upsilon_{A} \circ-d\left(\Upsilon_{A B}\right) \quad 3 \\
& \text { else } \quad A \vdash \Upsilon_{B} \quad \text { Ma } \Upsilon_{A} \circ \circ \Upsilon_{A B}, \Upsilon_{B} \circ \circ \Upsilon_{B}, \Upsilon_{A} \circ-A \quad \mid 3 \\
& \mathrm{~B} \vdash \Upsilon_{\mathrm{A}} \quad \mathrm{Mb} \quad \Upsilon_{\mathrm{B}} \circ \dot{\circ} \circ \Upsilon_{\mathrm{AB}}, \Upsilon_{\mathrm{A}} \circ \circ \Upsilon_{\mathrm{A}}, \Upsilon_{\mathrm{B}} \circ \dot{\circ} \mathrm{B} \quad 3 \\
& \text { else } \quad W \quad \Upsilon_{A} \circ \dot{\circ} \circ \Upsilon_{A B}, \Upsilon_{A} \circ \cdots \Upsilon_{B}, \Upsilon_{B} \circ \circ\left(\Upsilon_{A B}\right) \mid 4
\end{aligned}
$$


$(2,2,0,2)$

$$
\begin{array}{lll|l}
\Upsilon_{A} \bowtie \bar{\Upsilon} & \text { Ia } & \Upsilon_{A} \circ \circ \circ \Upsilon_{A}, 2 \times \Upsilon_{B} \circ \circ \Upsilon_{A B} & 3 \\
\Upsilon_{B} \bowtie \bar{\Upsilon} & \text { Ib } & \Upsilon_{B} \circ \circ \Upsilon_{B}, 2 \times \Upsilon_{A} \circ \circ \Upsilon_{A B} & 3 \\
\text { else } & W & \Upsilon_{A} \circ \circ \circ \Upsilon_{A B}, \Upsilon_{A B} \circ \circ \Upsilon_{B}, \Upsilon_{B} \circ \cdots \Upsilon_{A} & 4
\end{array}
$$

$(2,2,0,3)$

$$
\begin{array}{lll|l}
\Upsilon_{A} \Leftrightarrow \bar{\Upsilon} \wedge \Upsilon_{B} \Leftrightarrow \bar{\Upsilon} \wedge & n R 2 \times \Upsilon_{A} \circ \circ \Upsilon_{A B}, \Upsilon_{B} \circ \circ \Upsilon_{A B}, \Upsilon_{B} \circ \circ\left(\Upsilon_{A B}\right) & 4 \\
A \wedge \Upsilon_{B} \wedge B \wedge \Upsilon_{A} & \gg \Upsilon_{A B} \circ \circ \Upsilon_{A B} \cup(2,2,0,1) & +1 \\
\text { else } &
\end{array}
$$

$(2,2,0,4)$

$$
\begin{array}{llll|l}
\Upsilon_{A} \Leftrightarrow \bar{\Upsilon} \wedge \Upsilon_{B} \Leftrightarrow \bar{\Upsilon} & n R & 2 \times \Upsilon_{A} \circ \circ \circ \Upsilon_{A B}, 2 \times \Upsilon_{B} \circ \circ \Upsilon_{A B} & 4 \\
\text { else } & \ggg & \Upsilon_{A B} \circ \circ \Upsilon_{A B} \cup(2,2,0,2) & +1
\end{array}
$$

Reduction Bound 4 residual leaf compositions of subgroup $\left(1,0,1, \ell_{\mathrm{AB}}\right)+\left(1,0,2, \ell_{\mathrm{AB}}\right)+\left(2,0,1, \ell_{\mathrm{AB}}\right)+\left(2,0,2, \ell_{\mathrm{AB}}\right)$

$(1,0,1,1)$

$$
\begin{array}{lll|l}
\Upsilon_{\varnothing} \bowtie \bar{\Upsilon} & S & \bigcirc, \Upsilon_{A} \circ \circ \Upsilon_{A B} & 2 \\
\text { else } & W & \Upsilon_{A B} \circ \circ \Upsilon_{A}, \Upsilon_{\varnothing} \cdots \sigma\left(\Upsilon_{A}\right) & 3
\end{array}
$$

$(1,0,1,2)$

$$
\text { any topology I } \Upsilon_{A} \circ \circ \Upsilon_{A B}, \Upsilon_{A B} \circ \ddot{\circ} \circ \Upsilon_{\varnothing} \mid 3
$$

$(1,0,2,1)$

$$
\begin{aligned}
& \Upsilon_{\varnothing} \bowtie \bar{\Upsilon} \quad \text { I } \quad \Upsilon_{A} \circ \cdots \Upsilon_{A B}, \Upsilon_{\varnothing \varnothing \cdots} \cdots \Upsilon_{\varnothing} \mid 3 \\
& \text { else } \quad W \quad \Upsilon_{A} \circ \cdots \Upsilon_{\varnothing}, \Upsilon_{\varnothing} \circ \cdots \Upsilon_{A B} \mid 4
\end{aligned}
$$

$(1,0,2,2)$

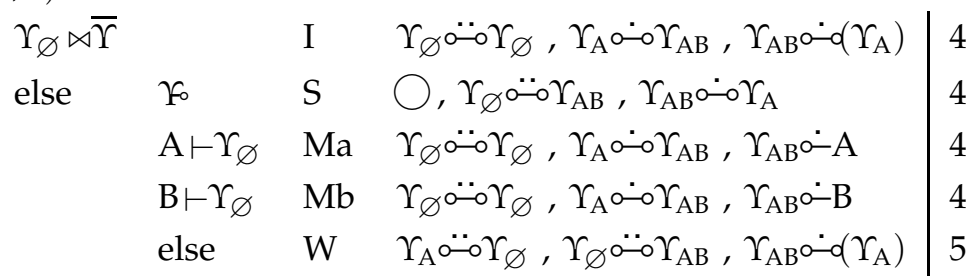

$(2,0,1,1)$

$$
\text { any topology I } \Upsilon_{\varnothing} \circ \cdots \Upsilon_{A}, \Upsilon_{A} \circ \circ \Upsilon_{A B} \mid 3
$$

$$
\begin{array}{lll|l}
\Upsilon_{\varnothing} \bowtie \bar{\Upsilon} & \mathrm{S} & \bigcirc, 2 \times \Upsilon_{A} \circ \dot{\circ} \Upsilon_{A B} & 3 \\
\text { else } & W & 2 \times \Upsilon_{A} \circ \cdots \Upsilon_{A B}, \Upsilon_{\varnothing} \circ-0\left(\Upsilon_{A B}\right) & 4
\end{array}
$$


$(2,0,2,1)$

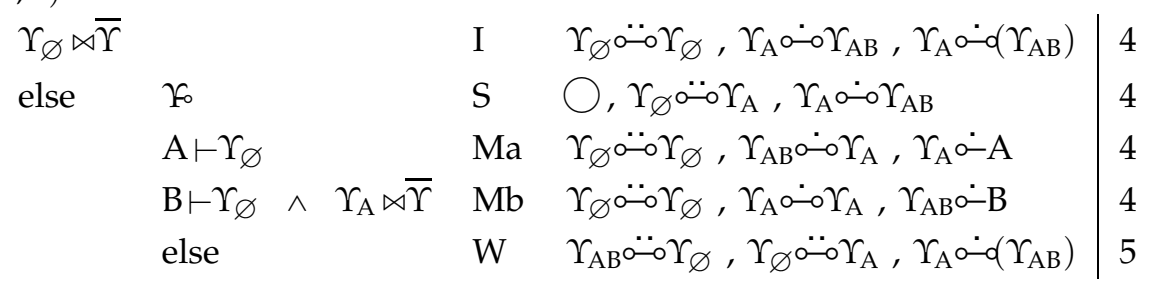

$(2,0,2,2)$

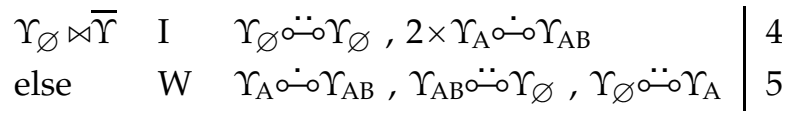

$(2,0,2,3)$

$\Upsilon_{\mathrm{A}} \Leftrightarrow \bar{\Upsilon} \wedge \Upsilon_{\varnothing} \Leftrightarrow \bar{\Upsilon} \wedge \mathrm{B} \vdash \Upsilon_{\varnothing}$

$\wedge A+r_{D} \wedge Y_{Q}$

$\mathrm{M} \Upsilon_{\varnothing} \because \ddot{\circ} \circ \Upsilon_{\varnothing}, 2 \times \Upsilon_{\mathrm{A}} \circ \sim \Upsilon_{\mathrm{AB}}, \Upsilon_{\mathrm{AB}} \circ \dot{\circ-\mathrm{B}} \mid 5$

else

$\ggg \Upsilon_{A B} \circ \circ \Upsilon_{A B} \cup(2,0,2,1)$

$+1$

\section{Enumeration of residual leaf compositions of Group 3}

Residual leaf compositions with four non-empty canonical subtrees. This includes 20 leaf compositions:

\begin{tabular}{c|cc|ccc}
\hline \multicolumn{6}{c}{ Reduction Bound 5 } \\
\hline Case 1 & \multicolumn{5}{c}{ Case 2 } \\
$\left(1,1,1, \ell_{\mathrm{AB}}\right)$ & $\left(1,1,2, \ell_{\mathrm{AB}}\right)$ & $\left(2,1,1, \ell_{\mathrm{AB}}\right)$ & $\left(2,1,2, \ell_{\mathrm{AB}}\right)$ & $\left(2,2,1, \ell_{\mathrm{AB}}\right)$ & $\left(2,2,2, \ell_{\mathrm{AB}}\right)$ \\
\hline$(1,1,1,1)$ & $(1,1,2,1)$ & $(2,1,1,1)$ & $(2,1,2,1)$ & $(2,2,1,1)$ & $(2,2,2,1)$ \\
$(1,1,1,2)$ & $(1,1,2,2)$ & $(2,1,1,2)$ & $(2,1,2,2)$ & $(2,2,1,2)$ & $(2,2,2,2)$ \\
& $(1,1,2,3)$ & $(2,1,1,3)$ & $(2,1,2,3)$ & $(2,2,1,3)$ & $(2,2,2,3)$ \\
& & & $(2,1,2,4)$ & $(2,2,1,4)$ & $(2,2,2,4)$ \\
\hline
\end{tabular}

$(1,1,1,1)$

$$
\begin{array}{llll|l}
\left(\Upsilon_{A} \smile \Upsilon_{A B}\right) \bowtie\left(\Upsilon_{B} \sim \Upsilon_{\varnothing}\right) & \text { Ia } & \Upsilon_{A} \circ \cdots \Upsilon_{A B}, \Upsilon_{B} \cdots \Upsilon_{\varnothing} \circ \Upsilon_{\varnothing} & 3 \\
\left(\Upsilon_{B} \sim \Upsilon_{A B}\right) \bowtie\left(\Upsilon_{A} \smile \Upsilon_{\varnothing}\right) \text { (else) } & \text { Ib } & \Upsilon_{B} \circ \cdots \Upsilon_{A B}, \Upsilon_{A} \cdots \cdots \Upsilon_{\varnothing} & 3
\end{array}
$$

$(1,1,1,2)$

$$
\begin{array}{lll|l}
\Upsilon_{\varnothing \bowtie \bar{\Upsilon}} & \mathrm{S} & \bigcirc, \Upsilon_{\mathrm{A}} \circ \dot{\circ} \circ \Upsilon_{\mathrm{AB}}, \Upsilon_{\mathrm{AB}} \circ \dot{\circ} \circ \Upsilon_{\mathrm{B}} & 3 \\
\text { else } & \mathrm{W} & \Upsilon_{\mathrm{A}} \circ \dot{\circ} \circ \Upsilon_{\mathrm{AB}}, \Upsilon_{\mathrm{AB}} \circ \dot{\circ} \circ \Upsilon_{\mathrm{B}}, \Upsilon_{\varnothing} \circ \ddot{\circ} \mathrm{o}\left(\Upsilon_{\mathrm{AB}}\right) & 4
\end{array}
$$

\begin{tabular}{|c|c|c|}
\hline & I & $\Upsilon_{\varnothing} \circ \ddot{\circ} \circ \Upsilon_{\varnothing}, \Upsilon_{A} \circ \cdots \Upsilon_{A B}, \Upsilon_{B} \circ \cdots\left(\Upsilon_{A B}\right)$ \\
\hline fo $_{0}$ & S1 & O, $\Upsilon_{\varnothing} \circ \ddot{\circ} \circ \Upsilon_{A}, \Upsilon_{B} \circ \cdots \Upsilon_{A B}$ \\
\hline $\mathrm{A} \vdash \Upsilon_{\varnothing}$ & Ma & $\Upsilon_{\varnothing} \circ \ddot{\circ} \circ \Upsilon_{\varnothing}, \Upsilon_{B} \circ \cdots \Upsilon_{A B}, \Upsilon_{A} \circ-A$ \\
\hline$f_{0}$ & $\mathrm{~S} 2$ & $\bigcirc, \Upsilon_{\varnothing} \circ \ddot{\circ} \circ \Upsilon_{B}, \Upsilon_{A} \circ \sim \Upsilon_{A B}$ \\
\hline $\mathrm{B} \vdash \Upsilon_{\varnothing}$ & $\mathrm{Mb}$ & $\Upsilon_{\varnothing} \circ \ddot{\circ} \circ \Upsilon_{\varnothing}, \Upsilon_{A} \circ \cdots \Upsilon_{A B}, \Upsilon_{B} \circ-B$ \\
\hline & $\mathrm{W}$ & $\Upsilon_{A} \circ \ddot{\circ} \circ \Upsilon_{\varnothing}, \Upsilon_{\varnothing} \circ \ddot{\circ} \circ \Upsilon_{B}, \Upsilon_{A B} \circ-0\left(\Upsilon_{A}\right)$ \\
\hline
\end{tabular}

$$
\begin{aligned}
& \Upsilon_{\varnothing \bowtie \bar{\Upsilon}} \\
& \text { else } \\
& \left(\Upsilon_{\mathrm{A}} \Leftrightarrow \Upsilon_{\varnothing}\right) \bowtie\left(\Upsilon_{\mathrm{B}} \smile \Upsilon_{\mathrm{AB}}\right) \\
& \text { I } \quad \Upsilon_{\varnothing} \because \ddot{\circ} \circ \Upsilon_{\varnothing}, \Upsilon_{A} \circ \cdots \Upsilon_{A B}, \Upsilon_{B} \circ-0\left(\Upsilon_{A B}\right) \mid 4 \\
& \left(\Upsilon_{\mathrm{B}} \Leftrightarrow \Upsilon_{\varnothing}\right) \bowtie\left(\Upsilon_{\mathrm{A}} \smile \Upsilon_{\mathrm{AB}}\right)
\end{aligned}
$$

$(1,1,2,2)$

$$
\begin{aligned}
& \Upsilon_{\varnothing} \bowtie \bar{\Upsilon} \quad \text { I } \quad \Upsilon_{\varnothing} \circ \ddot{\circ} \circ \Upsilon_{\varnothing}, \Upsilon_{A} \circ \cdots \Upsilon_{A B}, \Upsilon_{B} \circ \circ \Upsilon_{A B} \mid 4 \\
& \text { else } \quad W \quad \Upsilon_{A} \circ \ddot{\circ} \Upsilon_{\varnothing}, \Upsilon_{\varnothing} \circ \because \Upsilon_{A B}, \Upsilon_{A B} \circ \cdots \Upsilon_{B} \mid 5
\end{aligned}
$$


$(1,1,2,3)$

$$
\begin{aligned}
& \left(\Upsilon_{\varnothing} \Leftrightarrow \Upsilon_{A}\right) \Leftrightarrow \bar{\Upsilon} \wedge \mathrm{A} \vdash \Upsilon_{\varnothing} \wedge \mathrm{B} \vdash \Upsilon_{\varnothing} \quad \mathrm{Ma} \quad \Upsilon_{\varnothing} \circ{ }_{\circ} \circ \Upsilon_{\varnothing}, \Upsilon_{A} \circ \circ \Upsilon_{A B}, \Upsilon_{A B} \circ \circ \Upsilon_{B}, \Upsilon_{A B} \circ-A \mid 5 \\
& \left(\Upsilon_{\varnothing} \Leftrightarrow \Upsilon_{B}\right) \Leftrightarrow \bar{\Upsilon} \wedge \mathrm{B} \vdash \Upsilon_{\varnothing} \wedge \mathrm{A} \vdash \Upsilon_{\varnothing \varnothing} \quad \mathrm{Mb} \quad \Upsilon_{\varnothing} \circ \ddot{\circ}_{\circ} \Upsilon_{\varnothing}, \Upsilon_{A} \circ \dot{\circ} \circ \Upsilon_{A B}, \Upsilon_{A B} \circ \dot{\circ} \circ \Upsilon_{B}, \Upsilon_{A B} \circ \dot{-B} \quad 5 \\
& \text { else } \quad \ggg \Upsilon_{\mathrm{AB}} \circ \circ \Upsilon_{\mathrm{AB}} \cup(1,1,2,1) \quad \mid+1
\end{aligned}
$$

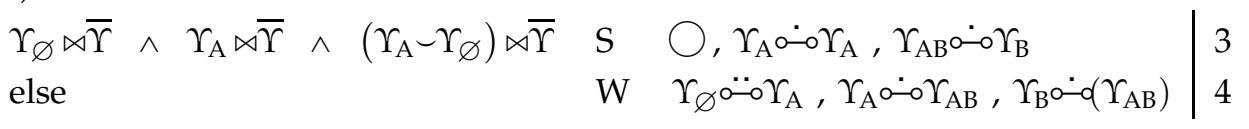

$(2,1,1,2)$

$$
\text { any topology I } \Upsilon_{A} \circ \cdots \Upsilon_{A B}, \Upsilon_{B} \circ \cdots \Upsilon_{A B}, \Upsilon_{A} \circ \cdots \Upsilon_{\varnothing} \mid 4
$$

$$
\begin{array}{lll|l}
\Upsilon_{A} \Leftrightarrow \bar{\Upsilon} \wedge \Upsilon_{\varnothing} \bowtie \bar{\Upsilon} & S & \bigcirc, 2 \times \Upsilon_{A} \circ \dot{\circ} \Upsilon_{A B}, \Upsilon_{A B} \circ \dot{\circ} \circ \Upsilon_{B} & 4 \\
\left(\Upsilon_{A} \bowtie \Upsilon_{\varnothing} \Leftrightarrow \hat{\Upsilon}\right. & & \\
\text { else } & \ggg & \Upsilon_{A B} \circ \dot{\circ} \circ \Upsilon_{A B} \cup(2,1,1,1) & +1
\end{array}
$$

$(2,1,2,1)$

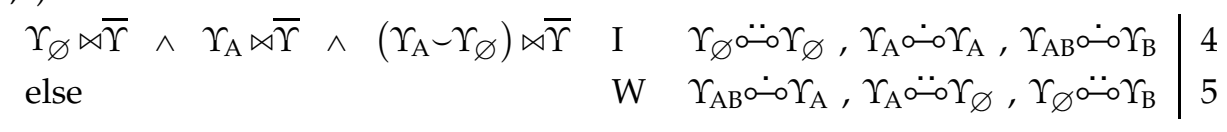

$(2,1,2,2)$

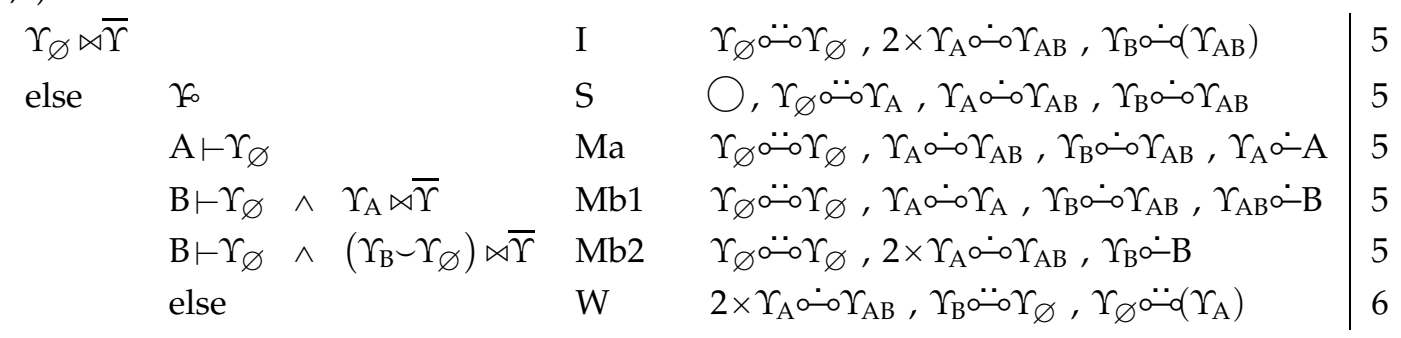

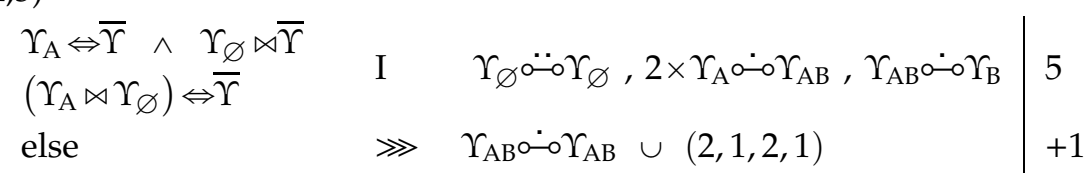

$$
\begin{aligned}
& \Upsilon_{\varnothing} \Leftrightarrow \bar{\Upsilon} \wedge \Upsilon_{\mathrm{A}} \Leftrightarrow \bar{\Upsilon} \bar{\Upsilon}
\end{aligned}
$$

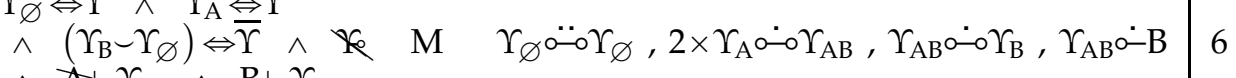

$$
\begin{aligned}
& \wedge A+\Upsilon_{\varnothing} \wedge \mathrm{B} \vdash \Upsilon_{\varnothing} \\
& \text { else } \\
& \ggg \Upsilon_{A B} \circ \circ \Upsilon_{A B} \cup(2,1,2,2) \\
& +1
\end{aligned}
$$


$(2,2,1,1)$

\begin{tabular}{|c|c|c|c|}
\hline$\Upsilon_{\mathrm{A}} \bowtie \bar{\Upsilon}$ & & Ia & $\Upsilon_{A} \circ \cdots \Upsilon_{A}, \Upsilon_{\varnothing} \circ \ddot{\circ} \circ \Upsilon_{B}, \Upsilon_{B} \circ \cdots \Upsilon_{A B}$ \\
\hline$\Upsilon_{B} \bowtie \bar{\Upsilon}$ & & $\mathrm{Ib}$ & $\Upsilon_{B} \circ \cdots \Upsilon_{B}, \Upsilon_{\varnothing} \circ \cdots \Upsilon_{A}, \Upsilon_{A} \circ \cdots \Upsilon_{A B}$ \\
\hline$\Upsilon_{\varnothing} \bowtie \bar{\Upsilon}$ & $\mathrm{A} \vdash \Upsilon_{\mathrm{B}}$ & $\mathrm{SMa}$ & $\bigcirc, \Upsilon_{A B} \circ \circ \Upsilon_{A}, \Upsilon_{B} \circ \circ \Upsilon_{B}, \Upsilon_{A} \circ-A$ \\
\hline & $\mathrm{B} \vdash \Upsilon_{\mathrm{A}}$ & $\mathrm{SMb}$ & $\bigcirc, \Upsilon_{A B} \circ \circ \Upsilon_{B}, \Upsilon_{A} \circ \circ \Upsilon_{A}, \Upsilon_{B} \circ-B$ \\
\hline else & & $\mathrm{W}$ & $\Upsilon_{A B} \circ \ddot{\circ} \circ \Upsilon_{A}, \Upsilon_{A} \circ \ddot{\circ} \circ \Upsilon_{B}, \Upsilon_{B} \cdots \ddot{\circ} \circ \Upsilon_{\varnothing}$ \\
\hline
\end{tabular}

$(2,2,1,2)$

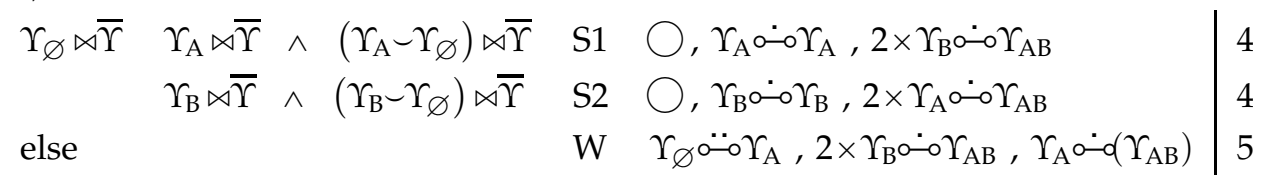

$(2,2,1,3)$

$$
\begin{aligned}
& \Upsilon_{\mathrm{A}} \Leftrightarrow \bar{\Upsilon} \wedge \Upsilon_{\mathrm{B}} \Leftrightarrow \bar{\Upsilon}
\end{aligned}
$$

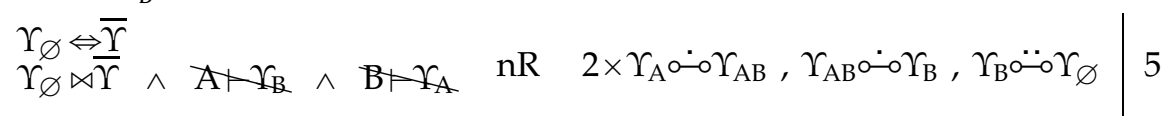

$$
\begin{aligned}
& \text { else } \\
& \ggg \Upsilon_{A B} \circ \circ \Upsilon_{A B} \cup(2,2,1,1) \\
& +1
\end{aligned}
$$

$(2,2,1,4)$

$$
\begin{aligned}
& \Upsilon_{\varnothing \bowtie \bar{\Upsilon} \wedge} \\
& \left\{\Upsilon_{\mathrm{A}} \Leftrightarrow \bar{\Upsilon} \vee\left(\Upsilon_{\mathrm{A}} \bowtie \Upsilon_{\varnothing}\right) \Leftrightarrow \bar{\Upsilon}\right\} \wedge \\
& \left\{\Upsilon_{B} \Leftrightarrow \bar{\Upsilon} \vee\left(\Upsilon_{B} \bowtie \Upsilon_{\varnothing}\right) \Leftrightarrow \bar{\Upsilon}\right\} \\
& \begin{array}{ll|l}
\mathrm{S} & \bigcirc, 2 \times \Upsilon_{\mathrm{A}} \circ \dot{\circ} \circ \Upsilon_{\mathrm{AB}}, 2 \times \Upsilon_{\mathrm{B}} \circ \dot{\circ} \circ \Upsilon_{\mathrm{AB}} & 5 \\
\ggg & \Upsilon_{\mathrm{AB}} \circ \dot{\circ} \circ \Upsilon_{\mathrm{AB}} \cup(2,2,1,2) & +1
\end{array}
\end{aligned}
$$

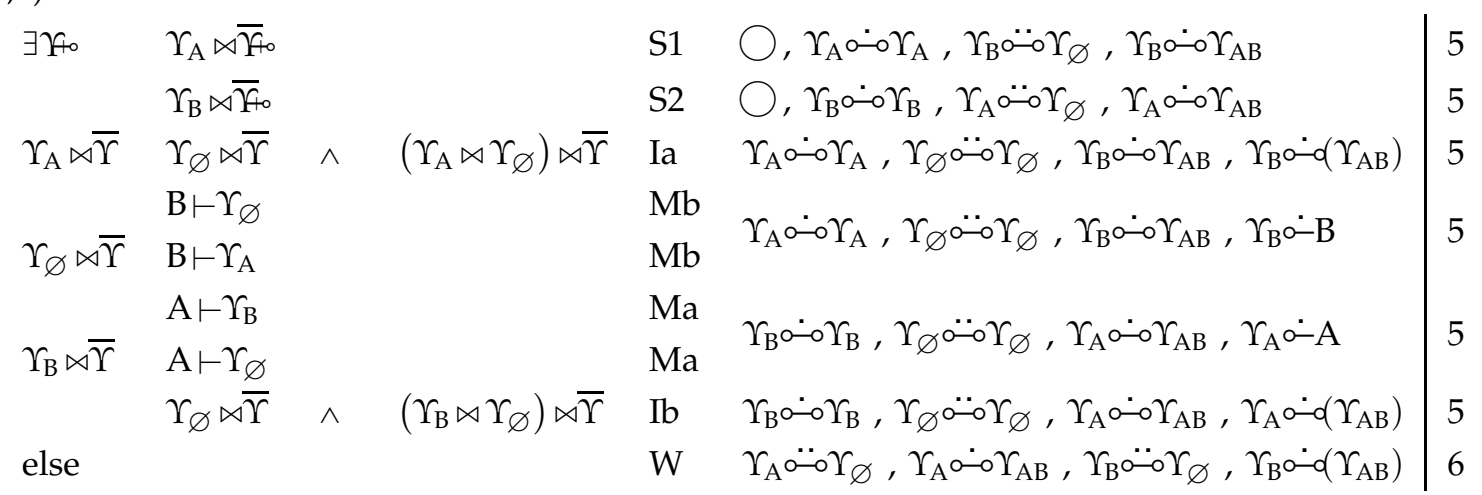

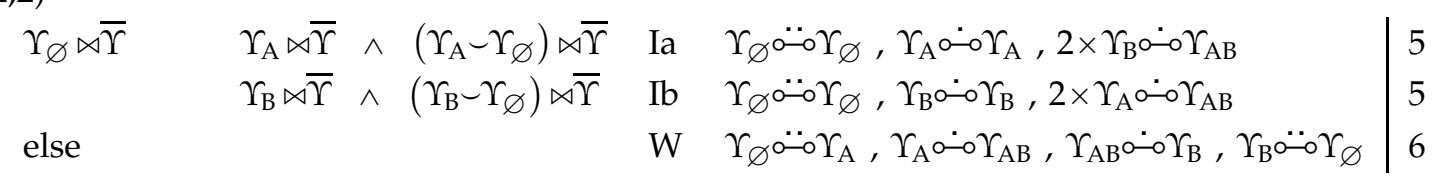


$(2,2,2,3)$

$$
\begin{aligned}
& \left\{\Upsilon_{\mathrm{A}} \Leftrightarrow \bar{\Upsilon} \vee\left(\Upsilon_{\mathrm{A}} \smile \Upsilon_{\varnothing}\right) \Leftrightarrow \bar{\Upsilon}\right\} \wedge \\
& \left\{\Upsilon_{B} \Leftrightarrow \bar{\Upsilon} \vee\left(\Upsilon_{B} \backsim \Upsilon_{\varnothing}\right) \Leftrightarrow \bar{\Upsilon}\right\} \\
& \Upsilon_{\varnothing} \bowtie \bar{\Upsilon} \\
& \text { fo } \\
& \text { I } \Upsilon_{\varnothing} \circ \ddot{\circ} \circ \Upsilon_{\varnothing}, 2 \times \Upsilon_{A} \circ \cdots \Upsilon_{A B}, \Upsilon_{B} \circ \dot{\circ} \circ \Upsilon_{A B}, \Upsilon_{B} \circ-0\left(\Upsilon_{A B}\right) \quad 6 \\
& \text { S } \bigcirc, \Upsilon_{\varnothing} \circ \circ \Upsilon_{A}, \Upsilon_{A} \circ \circ \Upsilon_{A B}, 2 \times \Upsilon_{A B} \circ \circ \Upsilon_{B} \quad 6 \\
& \Upsilon_{\mathrm{A}} \Leftrightarrow \bar{\Upsilon} \wedge \Upsilon_{\mathrm{B}} \Leftrightarrow \bar{\Upsilon} \wedge \Upsilon_{\varnothing} \Leftrightarrow \bar{\Upsilon} \\
& \mathrm{A} \vdash \Upsilon_{\varnothing} \\
& \text { Ma } \Upsilon_{\varnothing} \circ \circ \Upsilon_{\varnothing}, 2 \times \Upsilon_{B} \circ \circ \Upsilon_{A B}, \Upsilon_{A} \circ \cdots \Upsilon_{A B}, \Upsilon_{A} \circ \dot{-A} \quad 6 \\
& B \vdash \Upsilon_{\varnothing} \\
& \mathrm{Mb} \Upsilon_{\varnothing} \ddot{\circ} \circ \Upsilon_{\varnothing}, 2 \times \Upsilon_{A} \circ \cdots \Upsilon_{A B}, \Upsilon_{B} \circ \dot{\circ} \circ \Upsilon_{A B}, \Upsilon_{B} \circ-B \\
& \ggg \quad \Upsilon_{A B} \circ \circ \Upsilon_{A B} \cup(2,2,2,1)
\end{aligned}
$$

6

$(2,2,2,4)$

$$
\begin{aligned}
& \Upsilon_{\varnothing} \bowtie \bar{\Upsilon} \wedge \\
&\left\{\Upsilon_{A} \Leftrightarrow \bar{\Upsilon} \vee\right.\left.\left(\Upsilon_{A} \bowtie \Upsilon_{\varnothing}\right) \Leftrightarrow \bar{\Upsilon}\right\} \wedge \\
&\left\{\Upsilon_{B} \Leftrightarrow \bar{\Upsilon} \vee\left(\Upsilon_{B} \bowtie \Upsilon_{\varnothing}\right) \Leftrightarrow \bar{\Upsilon}\right\}
\end{aligned}
$$

else

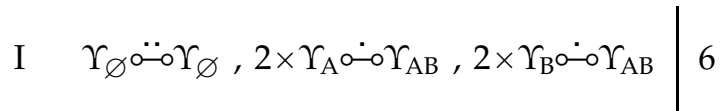

$$
\begin{aligned}
& \ggg \Upsilon_{A B} \circ-\circ \Upsilon_{A B} \cup(2,2,2,2) \\
& +1
\end{aligned}
$$

Non-reducible topologies of leaf composition $(2,2,2,3)$

(i) $\Upsilon\left(L_{\varnothing}\right)$ non-isolated

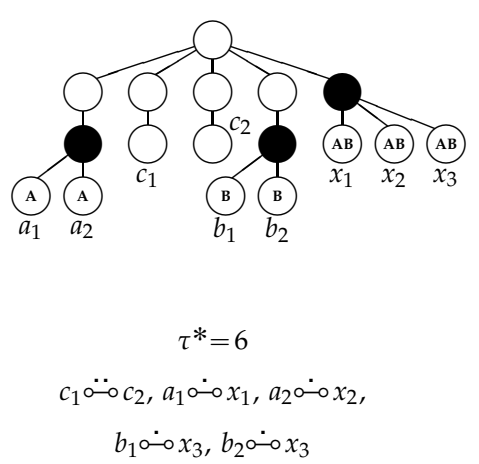

(ii) $\Upsilon\left(L_{\mathrm{A}} \cup L_{\varnothing}\right)$ isolated and solo leaf

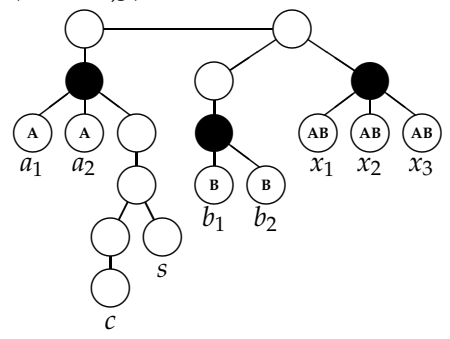

$$
\begin{gathered}
\tau^{*}=6 \\
\dot{s}, c \circ a_{1}, a_{2} \circ \circ x_{3}, \\
b_{1} \circ \sim x_{1}, b_{2} \circ \circ x_{2}
\end{gathered}
$$

(iii) tag mate at $\Upsilon^{+}\left(L_{\varnothing}\right)$

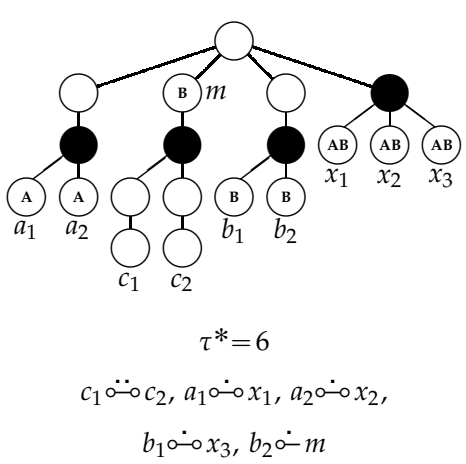

Fig. 12. All non-reducible topologies of leaf composition $(2,2,2,3)$ have a bad link between $\Upsilon\left(L_{\mathrm{AB}}\right)$ and $\Upsilon\left(L_{\mathrm{A}}\right)$ and a bad link between $\Upsilon\left(L_{\mathrm{AB}}\right)$ and $\Upsilon\left(L_{\mathrm{B}}\right)$. A symmetric case of (iii) is omitted. 
Topologies of leaf composition $(2,2,2,3)$ reducible to $(2,2,2,1)$

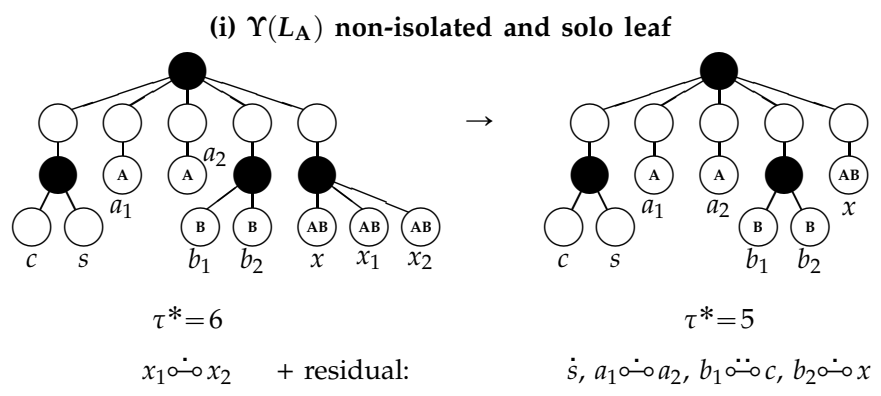

(ii) $\Upsilon\left(L_{\mathrm{A}}\right)$ and $\Upsilon\left(L_{\varnothing}\right)$ non-isolated

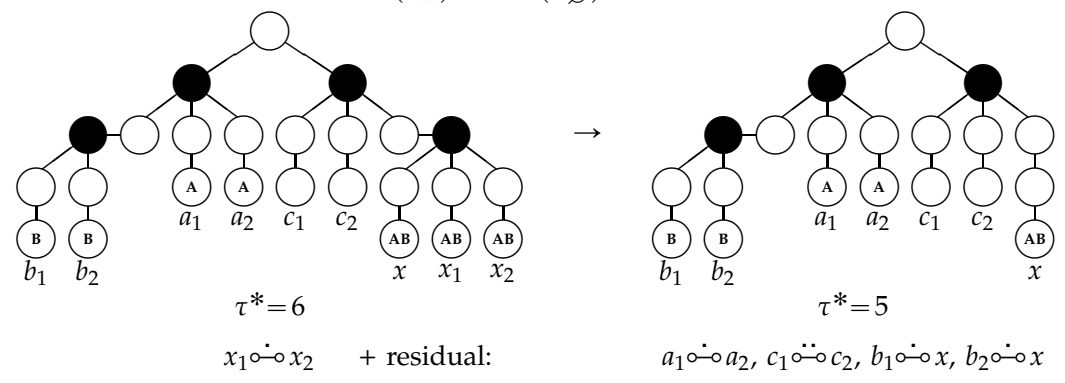

(iii) $\Upsilon\left(L_{\mathrm{A}}\right)$ non-isolated and B-mate at $\Upsilon^{+}\left(L_{\varnothing}\right)$

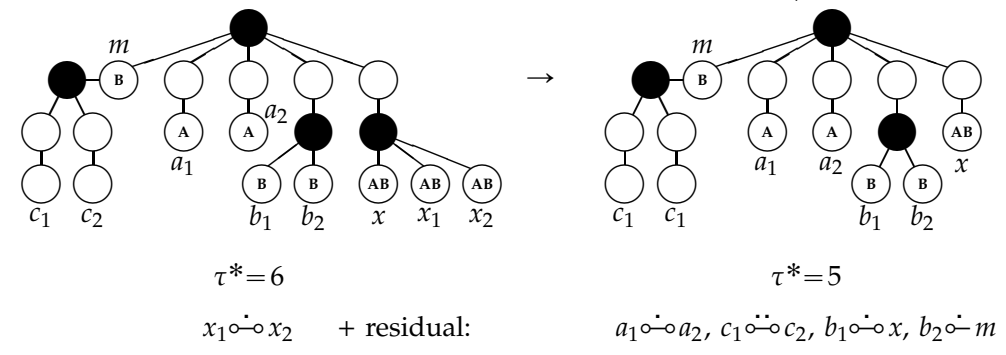

(iv) $\Upsilon\left(L_{\varnothing}\right)$ non-isolated and B-mate at $\Upsilon^{+}\left(L_{\mathrm{A}}\right)$

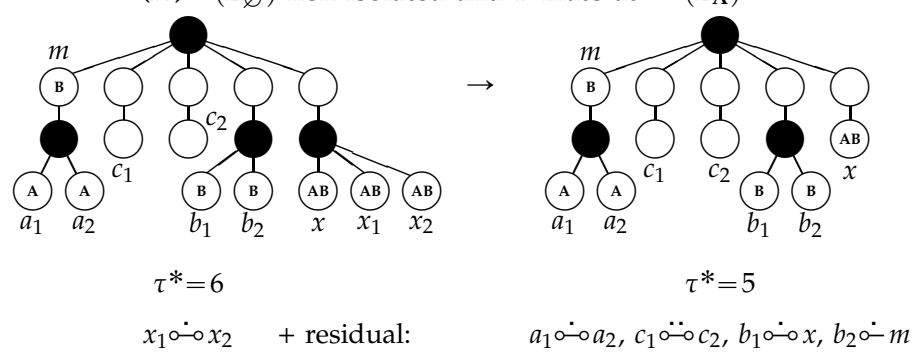

(v) all canonical subtrees isolated, no solo leaf, no tag mate at $\Upsilon^{+}\left(L_{\varnothing}\right)$

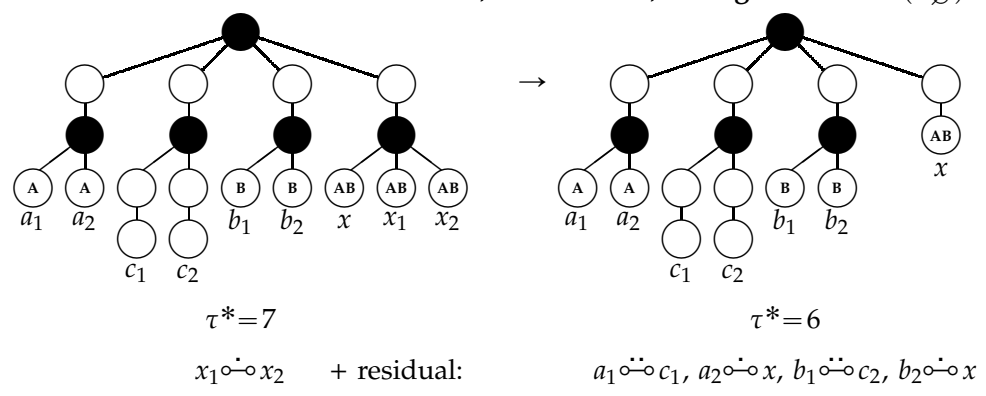

Fig. 13. There are five reducible topologies of leaf composition (2,2,2,3). Symmetric cases of (i), (ii), (iii) and (iv) are omitted. 